\title{
Approximation and symbolic calculus for Toeplitz algebras on the Bergman space
}

Daniel Suárez

\begin{abstract}
If $f \in L^{\infty}(\mathbb{D})$ let $T_{f}$ be the Toeplitz operator on the Bergman space $L_{a}^{2}$ of the unit disk $\mathbb{D}$. For a $C^{*}$-algebra $A \subset L^{\infty}(\mathbb{D})$ let $\mathfrak{T}(A)$ denote the closed operator algebra generated by $\left\{T_{f}: f \in A\right\}$. We characterize its commutator ideal $\mathfrak{C}(A)$ and the quotient $\mathfrak{T}(A) / \mathfrak{C}(A)$ for a wide class of algebras $A$. Also, for $n \geq 0$ integer, we define the $n$-Berezin transform $B_{n} S$ of a bounded operator $S$, and prove that if $f \in L^{\infty}(\mathbb{D})$ and $f_{n}=B_{n} T_{f}$ then $T_{f_{n}} \rightarrow T_{f}$.
\end{abstract}

\section{Introduction and preliminaries}

Suppose that $A$ is a $C^{*}$-algebra with unit. The commutator ideal $\mathfrak{C}$ is the closed bilateral ideal generated by the elements $[x, y]=x y-y x$, with $x, y \in A$. The quotient $A / \mathfrak{C}$ is a commutative $C^{*}$-algebra with unit, which by the Gelfand-Naimark Theorem is isometrically isomorphic to $C(M)$, the algebra of continuous functions on some compact Hausdorff space $M$. Following the arrows

$$
A \rightarrow A / \mathfrak{C} \stackrel{\simeq}{\longrightarrow} C(M)
$$

we can associate to every $x \in A$ a function $f_{x} \in C(M)$, which is the 'symbol' referred to in the title of the paper. Since the algebra $A$ is determined by $\mathfrak{C}$ and $C(M)$, the study of these two objects is an important tool for a better understanding of $A$. The possible advantages of this point of view are that $C(M)$ can be treated by topological methods, since it depends exclusively on the space $M$, and that $\mathfrak{C}$ is usually much smaller than $A$. Of course, the first step of this journey is to determine $\mathfrak{C}$ and $C(M)$. The whole process is known as abelianization, and it can be carried out for a much wider class

2000 Mathematics Subject Classification: Primary: 32A36; Secondary: 47B35.

Keywords: Bergman space, Toeplitz operator, commutator ideal and abelianization. 
of algebras than $C^{*}$-algebras. In particular, these ideas have being widely studied in the context of Toeplitz algebras acting on the Hardy space $H^{2}$ (see [18, pp. 339-392]). The literature shows some partial attempts to develop a similar scheme for Toeplitz algebras acting on the Bergman space $L_{a}^{2}=L_{a}^{2}(d A)$, where $d A$ is the normalized area measure on $\mathbb{D}$ (see $[14$, Ch. 4$]$ for a general discussion). We give below a brief summary of known results.

Let $\mathfrak{L}\left(L_{a}^{2}\right)$ be the algebra of bounded operators on $L_{a}^{2}$. If $\mathcal{B} \subset L^{\infty}(\mathbb{D})$ is a closed subalgebra, let $\mathfrak{T}(\mathcal{B})$ be the closed subalgebra of $\mathfrak{L}\left(L_{a}^{2}\right)$ generated by the Toeplitz operators $\left\{T_{a}: a \in \mathcal{B}\right\}$ and $\mathfrak{C}(\mathcal{B})$ be the commutator ideal of $\mathfrak{T}(\mathcal{B})$.

In [11] Coburn proved that $\mathfrak{C}(C(\overline{\mathbb{D}}))$ is the ideal of compact operators and $\mathfrak{T}(C(\overline{\mathbb{D}})) / \mathfrak{C}(C(\overline{\mathbb{D}}))$ is isomorphic to $C(\partial \mathbb{D})$. In [17] McDonald and Sundberg characterized the quotient $\mathfrak{T}(\mathcal{U}) / \mathfrak{C}(\mathcal{U})$, where $\mathcal{U}$ is the $C^{*}$-algebra in $L^{\infty}(\mathbb{D})$ generated by $H^{\infty}$. Later, the two papers by Axler and Zheng ([4], [5]) provided additional information on Coburn's and McDonald-Sundberg's theorems by giving characterizations of the respective commutator ideals in terms of the Berezin transform. We give precise statements of these results in Sections 6 and 7. In [20] the author showed that $\mathfrak{C}\left(L^{\infty}(\mathbb{D})\right)=\mathfrak{T}\left(L^{\infty}(\mathbb{D})\right)$. Despite these results, no systematic theory of abelianization has been given so far for Toeplitz algebras on the Bergman space. One of the purposes of this paper is to develop a general theory of abelianization for Toeplitz algebras $\mathfrak{T}(\mathcal{B})$, where $\mathcal{B}$ belongs to a special class of $C^{*}$-algebras in $L^{\infty}(\mathbb{D})$ that we call hyperbolic. Our main goal is to explain the underlying phenomenon that is apparently common to Coburn's and McDonald-Sundberg's theorems, and to apply it to other hyperbolic algebras.

Let $\mathcal{A} \subset L^{\infty}(\mathbb{D})$ be the algebra of functions on $\mathbb{D}$ that are uniformly continuous with respect to the pseudohyperbolic metric. If $n$ is a nonnegative integer, we define the $n$-Berezin transform $B_{n}: \mathfrak{L}\left(L_{a}^{2}\right) \rightarrow \mathcal{A}$. This is a linear operator, and we show that if $a \in L^{\infty}(\mathbb{D})$ and $a_{n}=B_{n} T_{a}$, then $T_{a_{n}}$ tends to $T_{a}$ in operator norm. In particular, the Toeplitz algebras associated to $L^{\infty}(\mathbb{D})$ and $\mathcal{A}$ coincide. This will allow us to reduce the study of $\mathfrak{T}(\mathcal{B})$ and $\mathfrak{C}(\mathcal{B})$ for some $C^{*}$-algebras $\mathcal{B} \subset L^{\infty}(\mathbb{D})$ that are not hyperbolic, to the case of hyperbolic algebras. Once the reduction is made, we can use the maximal ideal space of $\mathcal{A}$ as a powerful tool to describe $\mathfrak{C}(\mathcal{B})$ and $\mathfrak{T}(\mathcal{B}) / \mathfrak{C}(\mathcal{B})$. We begin fixing some notation.

For $z \in \mathbb{D}$ denote

$$
\varphi_{z}(\omega)=\frac{z-\omega}{1-\bar{z} \omega}
$$

The pseudohyperbolic metric on $\mathbb{D}$ is defined as $\rho(z, \omega)=\left|\varphi_{z}(\omega)\right|$. This metric is invariant under the action of $\operatorname{Aut}(\mathbb{D})$. Sometimes, especially in 
estimates involving the triangle inequality, it will be useful to use the hyperbolic metric

$$
h(z, \omega)=\log \frac{1+\rho(z, \omega)}{1-\rho(z, \omega)}, \quad z, \omega \in \mathbb{D}
$$

instead of $\rho$. The passage from one metric to the other is justified because $f(x)=\log \frac{1+x}{1-x}$ is a strictly increasing function of $x \in(0,1)$. For $z \in \mathbb{D}$, $r \in(0,1)$ and $s \in(0, \infty)$ write

$$
K(z, r)=\{\omega \in \mathbb{D}: \rho(z, \omega) \leq r\} \text { and } K_{h}(z, r)=\{\omega \in \mathbb{D}: h(z, \omega) \leq s\}
$$

for the closed pseudohyperbolic (resp. hyperbolic) disk of center $z$ and radius $r$ (resp. $s$ ).

Let $\mathcal{B} \subset L^{\infty}(\mathbb{D})$ be a closed subalgebra, where by algebra we always mean a unitary algebra. The maximal ideal space of $\mathcal{B}$ is

$$
M(\mathcal{B})=\{\alpha: \mathcal{B} \rightarrow \mathbb{C}: \alpha \text { is linear, multiplicative and } \alpha(1)=1\}
$$

provided with the weak $*$ topology induced by the dual space of $\mathcal{B}$. It is a compact Hausdorff space. We can look at a function $f \in \mathcal{B}$ as a continuous function on $M(A)$ via the Gelfand transform

$$
\hat{f}(\alpha)=\alpha(f) \quad(\alpha \in M(\mathcal{B})) .
$$

If $\mathcal{B} \subset C(\mathbb{D}) \cap L^{\infty}(\mathbb{D})$ separates points of $\mathbb{D}$ then evaluations at points of $\mathbb{D}$ are members of $M(\mathcal{B})$. So, $\mathbb{D}$ is naturally imbedded into $M(\mathcal{B})$, and $\hat{f}$ is an extension to the whole maximal space of the function $f$. Unless the contrary is stated we avoid writing the hat for the Gelfand transform and look at $f$ as a function on $M(\mathcal{B})$. The algebra

$$
\mathcal{A}=\left\{f \in L^{\infty}(\mathbb{D}): f \text { is uniformly continuous with respect to } \rho\right\}
$$

will be a major protagonist of this paper. It is $C^{*}$-algebra such that $\mathbb{D}$ is dense in $M(\mathcal{A})$. Indeed, there cannot be $\alpha \in M(\mathcal{A}) \backslash \overline{\mathbb{D}}$, because otherwise there is $f \in \mathcal{A}$ with $f(\alpha)=0$ while $|f| \geq \delta>0$ on $\mathbb{D}$ (since $\mathcal{A}$ is a $C^{*}$ algebra). Since such $f$ is invertible in $\mathcal{A}$, it is not in the maximal ideal Ker $\alpha$. Further information on $M(\mathcal{A})$ can be found in [8].

If $a \in L^{\infty}(\mathbb{D})$ let $M_{a}$ be the multiplication operator on $L^{2}(\mathbb{D})$ and $T_{a}$ be the Toeplitz operator on $L_{a}^{2}$. That is, $T_{a}=P_{+} M_{a}$, where $P_{+}: L^{2}(\mathbb{D}) \rightarrow L_{a}^{2}$ is the Bergman projection. It is clear that $\left\|M_{a}\right\|=\|a\|_{\infty}$ and $\left\|T_{a}\right\| \leq\|a\|_{\infty}$. A big difference with Toeplitz operators on the Hardy space $H^{2}$ is that the latter inequality is not always an equality, although we still have that $T_{a}=0$ 
only when $a=0$. For $z \in \mathbb{D}$, the 'change of variable operator' is given by $U_{z} f=\left(f \circ \varphi_{z}\right) \varphi_{z}^{\prime}$. That is,

$$
\left(U_{z} f\right)(\omega)=f\left(\varphi_{z}(\omega)\right) \frac{|z|^{2}-1}{(1-\bar{z} \omega)^{2}} .
$$

Is easy to prove that $U_{z} T_{a} U_{z}=T_{a \circ \varphi_{z}}$ for every $a \in L^{\infty}(\mathbb{D})$, and since $U_{z}$ is unitary and self-adjoint, then

$$
\left(T_{a_{1}} \ldots T_{a_{n}}\right)_{z}=\left(U_{z} T_{a_{1}} U_{z}\right) \ldots\left(U_{z} T_{a_{n}} U_{z}\right)=T_{a_{1} \circ \varphi_{z}} \ldots T_{a_{n} \circ \varphi_{z}}
$$

for $a_{1}, \ldots, a_{n} \in L^{\infty}(\mathbb{D})$. We will write

$$
S_{z}=U_{z} T_{a} U_{z} \quad \text { for } S \in \mathfrak{L}\left(L_{a}^{2}\right) .
$$

The paper is organized as follows. The main results are Theorems 5.7, 6.4 and 6.5. In Section 2 we introduce the $n$-Berezin transform of a bounded operator and study its basic properties. If $a \in L^{\infty}(\mathbb{D}), B_{n} T_{a}$ coincides with $B_{n}(a)$, the more familiar $n$-Berezin transform of a function. In Section 3 we study the maximal ideal space of $\mathcal{A}$ and use some of its features to define the notion of hyperbolic algebra. A characterization of these algebras is obtained in terms of interpolating sequences.

If $S \in \mathfrak{T}(\mathcal{B})$, where $\mathcal{B}$ is a hyperbolic algebra, we construct in Section 4 a continuous map $\Psi_{S}^{\mathcal{B}}$ from the maximal ideal space of $\mathcal{B}$ into $\mathfrak{T}(\mathcal{B})$, when provided with the strong operator topology, and study its interaction with the $n$-Berezin transform. We prove that $\Psi_{S}^{\mathcal{B}}$ is multiplicative as a function of $S$, which translates into a kind of asymptotic multiplicative behavior of $B_{n}$. This will be a fundamental tool for much of what follows.

Theorem 5.7 shows that $T_{B_{n}(a)}$ tends to $T_{a}$ for $a \in L^{\infty}(\mathbb{D})$. As a consequence we obtain that if $B_{n}(a)$ belongs to a hyperbolic algebra $\mathcal{B}$ for infinitely many values of $n$ then $T_{a} \in \mathfrak{T}(\mathcal{B})$. This argument will reduce the study of $\mathfrak{T}(C)$ for some non-hyperbolic algebras $C \subset L^{\infty}(\mathbb{D})$ to the hyperbolic case.

Theorem 6.4 gives a characterization of $\mathfrak{C}(\mathcal{B})$ and $\mathfrak{T}(\mathcal{B}) / \mathfrak{C}(\mathcal{B})$ when $\mathcal{B}$ is hyperbolic. If $S$ is a finite sum of finite products of Toeplitz operators with symbols in $L^{\infty}(\mathbb{D})$ and $\mathcal{B}$ is a hyperbolic algebra, Theorem 6.5 provides a necessary and sufficient condition for $S \in \mathfrak{T}(\mathcal{B})$ and $S \in \mathfrak{C}(\mathcal{B})$.

Section 7 is devoted to applications of the previous results. It is shown that the theorem of McDonald-Sundberg and part of Coburn's theorem are particular cases of Theorem 6.4. An example will be given to illustrate how Theorems 5.7 and 6.4 can be used to characterize $\mathfrak{C}(C)$ and $\mathfrak{T}(C) / \mathfrak{C}(C)$ for some $C^{*}$-algebras $C \subset L^{\infty}(\mathbb{D})$ that are not hyperbolic.

Finally, we give a partial result towards a possible characterization of the center of $\mathfrak{T}\left(L^{\infty}(\mathbb{D})\right) / \mathcal{K}$, where $\mathcal{K}$ denotes the ideal of compact operators. We finish the paper posing some open problems. 


\section{The $n$-Berezin transform.}

If $n$ is a nonnegative integer and $z \in \mathbb{D}$, the function

$$
K_{z}^{(n)}(\omega)=\frac{1}{(1-\bar{z} \omega)^{2+n}} \quad(\omega \in \mathbb{D})
$$

is the reproducing kernel of $z$ in the weighted Bergman space $L_{a}^{2}\left(d A_{n}\right)$, where $d A_{n}(\omega)=(n+1)\left(1-|\omega|^{2}\right)^{n} d A(\omega)$. The $n$-Berezin transform of an operator $S \in \mathfrak{L}\left(L_{a}^{2}\right)$ is defined as

$$
\left(B_{n} S\right)(z) \stackrel{\text { def }}{=}(n+1)\left(1-|z|^{2}\right)^{2+n} \sum_{j=0}^{n}\left(\begin{array}{l}
n \\
j
\end{array}\right)(-1)^{j}\left\langle S\left(\omega^{j} K_{z}^{(n)}\right), \omega^{j} K_{z}^{(n)}\right\rangle
$$

It is clear that $B_{n} S \in C^{\infty}(\mathbb{D})$ for every $S \in \mathfrak{L}\left(L_{a}^{2}\right)$. Using that

$$
\sum_{j=0}^{n}\left(\begin{array}{l}
n \\
j
\end{array}\right)(-1)^{j}|\omega|^{2 j}=\left(1-|\omega|^{2}\right)^{n}
$$

we see that if $S=T_{a}$, with $a \in L^{\infty}(\mathbb{D})$, then

$$
\begin{aligned}
\left(B_{n} a\right)(z) & \stackrel{\text { def }}{=}\left(B_{n} T_{a}\right)(z) \\
& =(n+1)\left(1-|z|^{2}\right)^{2+n} \sum_{j=0}^{n}\left(\begin{array}{c}
n \\
j
\end{array}\right)(-1)^{j} \int_{D} \frac{a(\omega)|\omega|^{2 j}}{|1-\bar{z} \omega|^{2(2+n)}} d A(\omega) \\
& =\int_{D} a(\omega) \frac{\left(1-|z|^{2}\right)^{2+n}}{|1-\bar{z} \omega|^{2(2+n)}}(n+1)\left(1-|\omega|^{2}\right)^{n} d A(\omega) \\
(2.2) & =\int_{D} a\left(\varphi_{z}(\zeta)\right)(n+1)\left(1-|\zeta|^{2}\right)^{n} d A(\zeta),
\end{aligned}
$$

where the last equality comes from the change of variables $\omega=\varphi_{z}(\zeta)$. Since $d A_{n}(\xi)$ is a probability measure that tends to concentrate its mass at 0 when $n \rightarrow \infty$, then $\left(B_{n} a\right)(z)$ is an average of $a$ satisfying $\left\|B_{n}(a)\right\|_{\infty} \leq\|a\|_{\infty}$ for all $a \in L^{\infty}(\mathbb{D})$. A straightforward calculation shows that $B_{n}$ maps $L^{\infty}(\mathbb{D})$ into $\mathcal{A}$ for every $n \geq 0$, and we will prove in Corollary 4.6 that the same holds for $\mathfrak{L}\left(L_{a}^{2}\right)$. The last expression in (2.2) clearly shows that $\left\|B_{n}(a)-a\right\|_{\infty} \rightarrow 0$ when $n \rightarrow \infty$ for every $a \in \mathcal{A}$. That is, the sequence $\left\{B_{n}\right\}$ works as an approximate identity for $\mathcal{A}$. In particular, $\lim _{n}\left\|T_{B_{n}(a)}-T_{a}\right\|=0$ for $a \in \mathcal{A}$.

The 0-Berezin transform of an operator is the usual Berezin transform, which has been extensively used in recent research (see for instance [2], [4], [5] and [19]). The $n$-Berezin transforms of functions (not necessarily bounded) were introduced by Berezin in [6]. Many of the results of this section were 
proved by Ahern, Flores and Rudin [2] for $n$-Berezin transforms of functions of several variables. However, the results here do not follow immediately from theirs, because there are a priori several ways to define $B_{n} S$ for $n \geq 1$ and $S \in \mathfrak{L}\left(L_{a}^{2}\right)$ so that (2.2) holds when $S=T_{a}$. If for instance $S \in$ $\mathfrak{L}\left(L_{a}^{2}\right) \cap \mathfrak{L}\left(L_{a}^{2}\left(d A_{n}\right)\right)$, then the usual Berezin transform of $S$ with respect to the weighted Bergman space $L_{a}^{2}\left(d A_{n}\right)$ is $\left(1-|z|^{2}\right)^{2+n}\left\langle S K_{z}^{(n)}, K_{z}^{(n)}\right\rangle_{d A_{n}}$, which differs from our definition of $B_{n} S$. It is precisely because of the results of this section (especially Proposition 2.4) that I convinced myself (and hopefully convince the reader) about (2.1) as the right definition of $B_{n} S$ for $S \in \mathfrak{L}\left(L_{a}^{2}\right)$.

Lemma 2.1 Let $S \in \mathfrak{L}\left(L_{a}^{2}\right)$ and $n \geq 0$. Then

$$
(n+2)\left(1-|z|^{2}\right) B_{n}\left(S-T_{\bar{\omega}} S T_{\omega}\right)(z)=(n+1) B_{n+1}\left(T_{1-\bar{\omega} z} S T_{1-\omega \bar{z}}\right)(z)
$$

for every $z \in \mathbb{D}$.

Proof. A simple rearrangement of terms gives

$$
\begin{gathered}
\sum_{j=0}^{n}\left(\begin{array}{c}
n \\
j
\end{array}\right)(-1)^{j}\left[\left\langle S\left(\omega^{j} K_{z}^{(n)}\right), \omega^{j} K_{z}^{(n)}\right\rangle-\left\langle S\left(\omega^{j+1} K_{z}^{(n)}\right), \omega^{j+1} K_{z}^{(n)}\right\rangle\right] \\
=\left\langle S K_{z}^{(n)}, K_{z}^{(n)}\right\rangle+(-1)^{n+1}\left\langle S\left(\omega^{n+1} K_{z}^{(n)}\right), \omega^{n+1} K_{z}^{(n)}\right\rangle \\
\quad+\sum_{j=1}^{n}\left[\left(\begin{array}{c}
n \\
j
\end{array}\right)+\left(\begin{array}{c}
n \\
j-1
\end{array}\right)\right](-1)^{j}\left\langle S\left(\omega^{j} K_{z}^{(n)}\right), \omega^{j} K_{z}^{(n)}\right\rangle \\
=\sum_{j=0}^{n+1}\left(\begin{array}{c}
n+1 \\
j
\end{array}\right)(-1)^{j}\left\langle S\left(\omega^{j} K_{z}^{(n)}\right), \omega^{j} K_{z}^{(n)}\right\rangle .
\end{gathered}
$$

Multiplying by $(n+2)(n+1)\left(1-|z|^{2}\right)^{3+n}$ and using that

$$
T_{1-\omega \bar{z}}\left(\omega^{j} K_{z}^{(n+1)}\right)=\omega^{j} K_{z}^{(n)},
$$

the above equality becomes (2.3).

Lemma $2.2 B_{n} S_{\alpha}=\left(B_{n} S\right) \circ \varphi_{\alpha}$ for every $n \geq 0, S \in \mathfrak{L}\left(L_{a}^{2}\right)$ and $\alpha \in \mathbb{D}$.

Proof. We shall prove the lemma by induction on $n$. The easy identity

$$
\left(1-\varphi_{\alpha}(\omega) \bar{z}\right)^{-1}=(1-\alpha \bar{z})^{-1}(1-\bar{\alpha} \omega)\left(1-\overline{\varphi_{\alpha}(z)} \omega\right)^{-1}
$$

implies that

$$
\left(U_{\alpha} K_{z}^{(0)}\right)(\omega)=\frac{\left(|\alpha|^{2}-1\right)}{(1-\bar{\alpha} \omega)^{2}\left(1-\varphi_{\alpha}(\omega) \bar{z}\right)^{2}}=\frac{\left(|\alpha|^{2}-1\right)}{(1-\alpha \bar{z})^{2}} K_{\varphi_{\alpha}(z)}^{(0)}(\omega) .
$$

Thus

$$
\left(B_{0} S_{\alpha}\right)(z)=\left(1-\left|\varphi_{\alpha}(z)\right|^{2}\right)^{2}\left\langle S K_{\varphi_{\alpha}(z)}^{(0)}, K_{\varphi_{\alpha}(z)}^{(0)}\right\rangle=\left(B_{0} S\right)\left(\varphi_{\alpha}(z)\right) .
$$

This takes care of $n=0$. 
The main tool for the inductive step will be formula (2.3), that we rewrite as

$$
\left(B_{n+1} S\right)(z)=c_{n}\left(1-|z|^{2}\right) B_{n}\left[T_{(1-\bar{\omega} z)^{-1}}\left(S-T_{\bar{\omega}} S T_{\omega}\right) T_{(1-\omega \bar{z})^{-1}}\right](z),
$$

where $c_{n}=(n+2) /(n+1)$. By (1.1) then

$$
\begin{aligned}
& T_{(1-\bar{\omega} z)^{-1}}\left(U_{\alpha} S U_{\alpha}-T_{\bar{\omega}} U_{\alpha} S U_{\alpha} T_{\omega}\right) T_{(1-\omega \bar{z})^{-1}} \\
& \quad=U_{\alpha} T_{\left(1-\overline{\varphi_{\alpha}(\omega)} z\right)^{-1}}\left[S-T_{\overline{\varphi_{\alpha}(\omega)}} S T_{\varphi_{\alpha}(\omega)}\right] T_{\left(1-\varphi_{\alpha}(\omega) \bar{z}\right)^{-1}} U_{\alpha}=J .
\end{aligned}
$$

Then (2.4) yields

$$
\begin{aligned}
& J=|1-\alpha \bar{z}|^{-2} U_{\alpha} T_{\left(1-\varphi_{\alpha}(z) \bar{\omega}\right)^{-1}}\left[T_{1-\alpha \bar{\omega}} S T_{1-\bar{\alpha} \omega}-T_{\bar{\alpha}-\bar{\omega}} S T_{\alpha-\omega}\right] T_{\left(1-\overline{\varphi_{\alpha}(z)} \omega\right)^{-1}} U_{\alpha} \\
& \quad=\frac{\left(1-|\alpha|^{2}\right)}{|1-\alpha \bar{z}|^{2}} U_{\alpha} T_{\left(1-\varphi_{\alpha}(z) \bar{\omega}\right)^{-1}}\left[S-T_{\bar{\omega}} S T_{\omega}\right] T_{\left(1-\overline{\varphi_{\alpha}(z)} \omega\right)^{-1}} U_{\alpha} .
\end{aligned}
$$

Hence,

$$
\begin{aligned}
& \left(B_{n+1} S_{\alpha}\right)(z)=c_{n}\left(1-|z|^{2}\right) B_{n}(J)(z) \\
& \quad=c_{n}\left(1-\left|\varphi_{\alpha}(z)\right|^{2}\right) B_{n}\left(U_{\alpha} T_{\left(1-\varphi_{\alpha}(z) \bar{\omega}\right)^{-1}}\left[S-T_{\bar{\omega}} S T_{\omega}\right] T_{\left(1-\overline{\varphi_{\alpha}(z)} \omega\right)^{-1}} U_{\alpha}\right)(z) \\
& \quad=c_{n}\left(1-\left|\varphi_{\alpha}(z)\right|^{2}\right) B_{n}\left(T_{\left(1-\varphi_{\alpha}(z) \bar{\omega}\right)^{-1}}\left[S-T_{\bar{\omega}} S T_{\omega}\right] T_{\left(1-\overline{\varphi_{\alpha}(z)} \omega\right)^{-1}}\right)\left(\varphi_{\alpha}(z)\right) \\
& \quad=B_{n+1}(S)\left(\varphi_{\alpha}(z)\right)
\end{aligned}
$$

where the first equality comes from (2.5) with $U_{\alpha} S U_{\alpha}$ instead of $S$, the second from (2.6), the third by inductive hypothesis and the last one from (2.5) with $\varphi_{\alpha}(z)$ instead of $z$.

Corollary 2.3 If $S \in \mathfrak{L}\left(L_{a}^{2}\right)$ and $n \geq 0$ then $\left\|B_{n} S\right\|_{\infty} \leq(n+1) 2^{n}\|S\|$.

Proof. Since $\left\|K_{z}^{(0)}\right\|=\left(1-|z|^{2}\right)^{-1}$ then

$$
\left|\left(B_{0} S\right)(z)\right|=\left(1-|z|^{2}\right)^{2}\left|\left\langle S\left(K_{z}^{(0)}\right), K_{z}^{(0)}\right\rangle\right| \leq\|S\| .
$$

Suppose that the corollary holds for $n$, and we shall see that it holds for $n+1$. By $(2.3)\left(B_{n+1} S\right)(0)=(n+2 / n+1) B_{n}\left(S-T_{\bar{\omega}} S T_{\omega}\right)(0)$. Thus

$$
\begin{aligned}
\left|\left(B_{n+1} S\right)(0)\right| & \leq \frac{n+2}{n+1}\left(\left\|B_{n} S\right\|_{\infty}+\left\|B_{n}\left(T_{\bar{\omega}} S T_{\omega}\right)\right\|_{\infty}\right) \\
& \leq \frac{n+2}{n+1}\left((n+1) 2^{n}\|S\|+(n+1) 2^{n}\left\|T_{\bar{\omega}} S T_{\omega}\right\|\right) \\
& \leq(n+2) 2^{n+1}\|S\| .
\end{aligned}
$$

Replacing $S$ by $U_{z} S U_{z}$ the result follows from Lemma 2.2. 
The (conformally) invariant Laplacian is $\tilde{\Delta}=\left(1-|z|^{2}\right)^{2} 4 \partial \bar{\partial}$, where $\partial$ and $\bar{\partial}$ are the traditional Cauchy-Riemann operators. So, when $f$ is analytic on $\mathbb{D}, \partial f=f^{\prime}, \partial \bar{f}=0, \bar{\partial} \bar{f}=\overline{f^{\prime}}$ and $\bar{\partial} f=0$. It is easy to check that $(\tilde{\Delta} f) \circ \psi=\tilde{\Delta}(f \circ \psi)$ for every $\psi \in \operatorname{Aut}(\mathbb{D})$.

Proposition 2.4 Let $S \in \mathfrak{L}\left(L_{a}^{2}\right)$ and $n \geq 0$. Then

$$
\tilde{\Delta} B_{n} S=4(n+1)(n+2)\left(B_{n} S-B_{n+1} S\right) .
$$

Proof. By Lemma 2.2 and the conformal invariance of $\tilde{\Delta}$ it is enough to prove that the equality holds at $z=0$. Using the mentioned properties of $\partial$ and $\bar{\partial}$, a tedious but straightforward calculation gives

$$
\begin{aligned}
\tilde{\Delta}[(1 & \left.\left.-|z|^{2}\right)^{n+2}\left\langle S\left(\omega^{j} K_{z}^{(n)}\right), \omega^{j} K_{z}^{(n)}\right\rangle\right](0) \\
& =4(n+2)\left(-\left\langle S \omega^{j}, \omega^{j}\right\rangle+(n+2)\left\langle S \omega^{j+1}, \omega^{j+1}\right\rangle\right)
\end{aligned}
$$

for every $0 \leq j \leq n$. So, writing $X_{j}=(-1)^{j}\left\langle S \omega^{j}, \omega^{j}\right\rangle$, we have

$$
\begin{aligned}
& \tilde{\Delta}\left(B_{n} S\right)(0)=4(n+1)(n+2) \sum_{j=0}^{n}\left(\begin{array}{l}
n \\
j
\end{array}\right)\left[-X_{j}-(n+2) X_{j+1}\right] \\
& =4(n+1)(n+2)\left\{-X_{0}-(n+2) X_{n+1}-\sum_{j=1}^{n}\left[\left(\begin{array}{c}
n \\
j
\end{array}\right)+(n+2)\left(\begin{array}{c}
n \\
j-1
\end{array}\right)\right] X_{j}\right\} .
\end{aligned}
$$

On the other hand,

$$
\left(B_{n} S-B_{n+1} S\right)(0)=-(n+2) X_{n+1}+\sum_{j=0}^{n}\left[(n+1)\left(\begin{array}{c}
n \\
j
\end{array}\right)-(n+2)\left(\begin{array}{c}
n+1 \\
j
\end{array}\right)\right] X_{j} .
$$

A comparison of the coefficients for each $X_{j}$ gives the result.

Corollary 2.5 If $S \in \mathfrak{L}\left(L_{a}^{2}\right)$ and $n \geq 1$ then

$$
B_{n} S=\left(1-\frac{\tilde{\Delta}}{4 n(n+1)}\right) B_{n-1} S
$$

and

$$
B_{n} S=G_{n}(\tilde{\Delta}) B_{0} S
$$

where

$$
G_{n}(\lambda)=\prod_{k=1}^{n}\left(1-\frac{\lambda}{4 k(k+1)}\right) .
$$

Proof. Formula (2.9) is a rewriting of (2.7), while (2.10) follows immediately from (2.9). 
Lemma 2.6 If $S \in \mathfrak{L}\left(L_{a}^{2}\right)$ and $n \geq 0$ then $\tilde{\Delta} B_{0}\left(B_{n} S\right)=B_{0} \tilde{\Delta}\left(B_{n} S\right)$.

Proof. If $f=B_{n} S$, Corollary 2.3 and (2.7) imply that $f$ and $\tilde{\Delta} f$ are bounded. Hence, Lemma 1 of [1] says that $\tilde{\Delta} B_{0} f=B_{0} \tilde{\Delta} f$.

Corollary 2.7 Let $S \in \mathfrak{L}\left(L_{a}^{2}\right)$ and $k, j \geq 0$. Then $\left(B_{k} B_{j}\right)(S)=\left(B_{j} B_{k}\right)(S)$.

Proof. Combine (2.10) with the previous lemma.

\section{Algebras related to the maximal ideal space of $\mathcal{A}$}

For the next two subsections, if $E \subset M(\mathcal{A})$ then $\bar{E}$ denotes the closure of $E$ in the space $M(\mathcal{A})$.

Since the $M(\mathcal{A})$-topology agrees with the Euclidean topology on $\mathbb{D}, \bar{E}$ has the same meaning in both topologies when $E \subset r \mathbb{D}$ for some $0<r<1$. Later on, we will have to distinguish between closures in different spaces. A sequence $\left\{z_{n}\right\} \subset \mathbb{D}$ is separated if $\rho\left(z_{n}, z_{k}\right) \geq \delta>0$ for $n \neq k$.

\subsection{One-to-one maps from $\mathbb{D}$ into $M(\mathcal{A})$}

Lemma 3.1 Let $E, F \subset \mathbb{D}$. Then $\bar{E} \cap \bar{F}=\emptyset$ if and only if $\rho(E, F)>0$.

Proof. If $\bar{E} \cap \bar{F}=\emptyset$ then there is $f \in \mathcal{A}$ such that $f \equiv 1$ on $E$ and $f \equiv 0$ on $F$. The uniform $\rho$-continuity of $f$ implies that

$$
\rho(E, F)=\rho(\bar{E} \cap \mathbb{D}, \bar{F} \cap \mathbb{D})>0 .
$$

Now suppose that $\rho(E, F) \geq \alpha>0$ and consider the function

$$
f(z)= \begin{cases}1 & \text { if } \rho(z, E) \leq \alpha / 2 \\ 0 & \text { if } \rho(z, E)>\alpha / 2\end{cases}
$$

Simple estimates show that $B_{n}(f) \rightarrow 1$ uniformly on $\{z: \rho(z, E)<\alpha / 4\}$ and $B_{n}(f) \rightarrow 0$ uniformly on $\{z: \rho(z, F)<\alpha / 4\}$. Since $B_{n}(f) \in \mathcal{A}$, it separates $\bar{E}$ from $\bar{F}$ for $n$ big enough, showing that they are disjoint.

Let $x \in M(\mathcal{A})$ and suppose that $\left(z_{\alpha}\right)$ is a net in $\mathbb{D}$ that tends to $x$. We can think of $\left(\varphi_{z_{\alpha}}\right)$ as a net in the product space $M(\mathcal{A})^{\mathbb{D}}$. By compactness there is a convergent subnet $\left(\varphi_{z_{\alpha_{\beta}}}\right)$, meaning that there is some function $\varphi: \mathbb{D} \rightarrow M(\mathcal{A})$ such that $f \circ \varphi_{z_{\alpha_{\beta}}} \rightarrow f \circ \varphi$ pointwise on $\mathbb{D}$ for every $f \in \mathcal{A}$.

We aim to show that the whole net $\left(z_{\alpha}\right)$ tends to $\varphi$ and that $\varphi$ does not depend on the net. So, suppose that $\left(\omega_{\gamma}\right)$ is another net in $\mathbb{D}$ converging to $x$ such that $\varphi_{\omega_{\gamma}}$ tends to some $\psi \in M(\mathcal{A})^{\mathbb{D}}$. If $\varphi \neq \psi$ there is 
some $\xi \in \mathbb{D}$ such that $\varphi(\xi) \neq \psi(\xi)$. Then there are closed disjoint neighborhoods $U, V \subset M(\mathcal{A})$ of $\varphi(\xi)$ and $\psi(\xi)$, respectively. Since $\varphi_{z_{\alpha_{\beta}}}(\xi) \rightarrow \varphi(\xi)$ and $\varphi_{\omega_{\gamma}}(\xi) \rightarrow \psi(\xi)$, there are tails of both nets satisfying

$$
E=\left\{\varphi_{z_{\alpha_{\beta}}}(\xi): \beta \geq \beta_{0}\right\} \subset U \text { and } F=\left\{\varphi_{\omega_{\gamma}}(\xi): \gamma \geq \gamma_{0}\right\} \subset V .
$$

By Lemma 3.1 then $\rho(E, F) \geq \rho(U \cap \mathbb{D}, V \cap \mathbb{D})>0$. Since for every $z, \omega \in \mathbb{D}$ there is a constant $c_{\xi}>0$ such that

$$
\rho\left(\varphi_{z}(\xi), \varphi_{\omega}(\xi)\right)<c_{\xi} \rho(z, \omega)
$$

then

$$
\rho(E, F) \leq c_{\xi} \inf \left\{\rho\left(z_{\alpha_{\beta}}, \omega_{\gamma}\right): \beta \geq \beta_{0}, \gamma \geq \gamma_{0}\right\}=0,
$$

where the last equality holds because both nets $\left(z_{\alpha_{\beta}}\right)$ and $\left(\omega_{\gamma}\right)$ tend to $x$. We obtain a contradiction and consequently $\varphi=\psi$. The map $\varphi$ will be denoted $\varphi_{x}$, and notice that $\varphi_{x}(0)=\lim \varphi_{z_{\alpha}}(0)=\lim z_{\alpha}=x$.

The following lemma is in [20, Lemma 2.1].

Lemma 3.2 Let $\mathcal{S}$ be a separated sequence and $0<\sigma<1$. Then there is a finite decomposition $\mathcal{S}=\mathcal{S}_{1} \cup \ldots \cup \mathcal{S}_{N}$ such that for every $1 \leq j \leq N$ : $\rho(z, \omega)>\sigma$ for all $z \neq \omega$ in $\mathcal{S}_{j}$.

Lemma 3.3 Every $x \in M(\mathcal{A})$ is in the closure of some separated sequence.

Proof. Suppose that $x \in M(\mathcal{A})$ and let $\left(\omega_{\alpha}\right)$ be a net in $\mathbb{D}$ such that $\omega_{\alpha} \rightarrow x$. Take a separated sequence $\mathcal{S}$ such that $\rho(z, \mathcal{S})<1 / 8$ for every $z \in \mathbb{D}$, and for each $\omega_{\alpha}$ pick some $z_{\alpha}$ in $\mathcal{S}$ such that $\rho\left(z_{\alpha}, \omega_{\alpha}\right)<1 / 8$ for every $\alpha$. Therefore there is $\xi_{\alpha} \in 8^{-1} \mathbb{D}$ so that $\omega_{\alpha}=\varphi_{z_{\alpha}}\left(\xi_{\alpha}\right)$. Taking subnets we can assume that $\xi_{\alpha} \rightarrow \xi$ with $|\xi| \leq 1 / 8$. We claim that $\varphi_{z_{\alpha}}(\xi)$ tends to $x$. Indeed, if $f \in \mathcal{A}$ then

$$
\left|f\left(\varphi_{z_{\alpha}}(\xi)\right)-f(x)\right| \leq\left|f\left(\varphi_{z_{\alpha}}(\xi)\right)-f\left(\varphi_{z_{\alpha}}\left(\xi_{\alpha}\right)\right)\right|+\left|f\left(\omega_{\alpha}\right)-f(x)\right|,
$$

where the first summand tends to 0 because $\rho\left(\varphi_{z_{\alpha}}(\xi), \varphi_{z_{\alpha}}\left(\xi_{\alpha}\right)\right)=\rho\left(\xi, \xi_{\alpha}\right) \rightarrow 0$, and the second summand tends to 0 because $\omega_{\alpha} \rightarrow x$. Thus, $x$ is in the closure of the sequence $\mathcal{T}=\left\{\varphi_{z_{n}}(\xi): z_{n} \in \mathcal{S}\right\}$. By Lemma 3.2 we can split $\mathcal{S}=\mathcal{S}_{1} \cup \ldots \cup \mathcal{S}_{N}$, where for each $1 \leq j \leq N, \rho\left(z_{1}, z_{2}\right)>1 / 2$ when $z_{1}, z_{2} \in \mathcal{S}_{j}$ are different. We also have the corresponding decomposition $\mathcal{T}=\mathcal{T}_{1} \cup \ldots \cup \mathcal{T}_{N}$, where $\mathcal{T}_{j}=\left\{\varphi_{z}(\xi): z \in \mathcal{S}_{j}\right\}$. Hence, there is at least one $j_{0}$ such that $x$ is in the closure of $\mathcal{T}_{j_{0}}$. The lemma will follow if we show that $\mathcal{T}_{j_{0}}$ is a separated sequence. If $z_{1}, z_{2} \in \mathcal{S}_{j_{0}}$ are different then

$$
\begin{aligned}
\rho\left(z_{1}, z_{2}\right) & \leq \rho\left(z_{1}, \varphi_{z_{1}}(\xi)\right)+\rho\left(\varphi_{z_{1}}(\xi), \varphi_{z_{2}}(\xi)\right)+\rho\left(\varphi_{z_{2}}(\xi), z_{2}\right) \\
& =2|\xi|+\rho\left(\varphi_{z_{1}}(\xi), \varphi_{z_{2}}(\xi)\right) .
\end{aligned}
$$

So, $\rho\left(\varphi_{z_{1}}(\xi), \varphi_{z_{2}}(\xi)\right) \geq(1 / 2)-2|\xi| \geq 1 / 4$, proving our claim. 
Lemma 3.4 Let $\left(z_{\alpha}\right)$ be a net in $\mathbb{D}$ converging to $x \in M(\mathcal{A})$. Then

(i) $\varphi_{x}$ is a continuous one-to-one map from $\mathbb{D}$ into $M(\mathcal{A})$,

(ii) $f \circ \varphi_{x} \in \mathcal{A}$ for every $f \in \mathcal{A}$,

(iii) $f \circ \varphi_{z_{\alpha}} \rightarrow f \circ \varphi_{x}$ uniformly on compact sets of $\mathbb{D}$ for every $f \in \mathcal{A}$.

Proof. Suppose that $\omega \in \mathbb{D}$ and $f \in \mathcal{A}$. Given $\varepsilon>0$ there is $\delta>0$ such that $|f(u)-f(v)|<\varepsilon$ if $\rho(u, v)<\delta$. Take $\omega_{1} \in K(\omega, \delta)$. Since $\rho\left(\varphi_{z_{\alpha}}\left(\omega_{1}\right), \varphi_{z_{\alpha}}(\omega)\right)=\rho\left(\omega_{1}, \omega\right)<\delta$ then $\left|f\left(\varphi_{z_{\alpha}}\left(\omega_{1}\right)\right)-f\left(\varphi_{z_{\alpha}}(\omega)\right)\right|<\varepsilon$ for every $\alpha$. Then

$$
\begin{aligned}
\mid f\left(\varphi_{x}\left(\omega_{1}\right)\right)- & f\left(\varphi_{x}(\omega)\right) \mid \\
\leq \mid & f\left(\varphi_{x}\left(\omega_{1}\right)\right)-f\left(\varphi_{z_{\alpha}}\left(\omega_{1}\right)\right)|+| f\left(\varphi_{z_{\alpha}}\left(\omega_{1}\right)\right)-f\left(\varphi_{z_{\alpha}}(\omega)\right) \mid \\
& \quad+\left|f\left(\varphi_{z_{\alpha}}(\omega)\right)-f\left(\varphi_{x}(\omega)\right)\right| \\
\leq & f\left(\varphi_{x}\left(\omega_{1}\right)\right)-f\left(\varphi_{z_{\alpha}}\left(\omega_{1}\right)\right)|+| f\left(\varphi_{z_{\alpha}}(\omega)\right)-f\left(\varphi_{x}(\omega)\right) \mid+\varepsilon
\end{aligned}
$$

for every $\alpha$. Taking limits in $\alpha$ we get $\left|f\left(\varphi_{x}\left(\omega_{1}\right)\right)-f\left(\varphi_{x}(\omega)\right)\right| \leq \varepsilon$ when $\rho\left(\omega_{1}, \omega\right)<\delta$. This proves the continuity of $\varphi_{x}$ and (ii).

To prove that $\varphi_{x}$ is one-to-one, for an arbitrary $0<r<1$ we will construct a function $f \in \mathcal{A}$ (depending on $r$ ) such that $\left(f \circ \varphi_{x}\right)(\omega)=\omega$ when $|\omega|<r$. It is convenient to deal with the hyperbolic metric $h$ instead of $\rho$. Write $s=\log \frac{1+r}{1-r}$. By Lemma 3.2 there is a sequence $\left\{z_{n}\right\}$ in $\mathbb{D}$ whose closure contains $x$ and such that $h\left(z_{n}, z_{m}\right)>5 s$ if $n \neq m$. Therefore

$$
h\left(K_{h}\left(z_{n}, 2 s\right), K_{h}\left(z_{m}, 2 s\right)\right) \geq s \text { if } n \neq m .
$$

Take $g \in C(\mathbb{D})$ such that $g(\omega)=\omega$ if $h(\omega, 0)<s$ (i.e.: if $|\omega|<r$ ) and $g(\omega)=0$ if $h(\omega, 0)>2 s$. Thus $g \circ \varphi_{z_{n}}$ is supported in $K_{h}\left(z_{n}, 2 s\right)$ and

$$
f=\sum_{n \geq 1}\left(g \circ \varphi_{z_{n}}\right) \in C(\mathbb{D}) .
$$

Since $g$ is uniformly continuous with respect to the Euclidean metric then it is $h$-uniformly continuous. Hence, given $\varepsilon>0$ there is $\delta$, with $0<\delta<s / 2$, such that

$$
\left|g\left(\xi_{1}\right)-g\left(\xi_{2}\right)\right|<\varepsilon \text { if } h\left(\xi_{1}, \xi_{2}\right)<\delta .
$$

Let $\omega_{1}, \omega_{2} \in \mathbb{D}$ such that $h\left(\omega_{1}, \omega_{2}\right)<\delta$. By $(3.1) K_{h}\left(\omega_{1}, \delta\right)$ cuts at most one of the disks $K_{h}\left(z_{n}, 2 s\right)$. If it doesn't cut any, then $f\left(\omega_{1}\right)=f\left(\omega_{2}\right)=0$. If it cuts $K_{h}\left(z_{n_{0}}, 2 s\right)$, then $f\left(\omega_{1}\right)-f\left(\omega_{2}\right)=g\left(\varphi_{z_{n_{0}}}\left(\omega_{1}\right)\right)-g\left(\varphi_{z_{n_{0}}}\left(\omega_{2}\right)\right)$, and since $h\left(\varphi_{z_{n_{0}}}\left(\omega_{1}\right), \varphi_{z_{n_{0}}}\left(\omega_{2}\right)\right)=h\left(\omega_{1}, \omega_{2}\right)<\delta$ then (3.2) says that $\left|f\left(\omega_{1}\right)-f\left(\omega_{2}\right)\right|<\varepsilon$. Thus $f \in \mathcal{A}$. 
If $k$ is any positive integer and $|\omega|<r$ then $h(0, \omega)<s$ and $\varphi_{z_{k}}(\omega) \in$ $K_{h}\left(z_{k}, s\right)$. So, (3.1) and the inclusion: $\operatorname{supp}\left(g \circ \varphi_{z_{n}}\right) \subset K_{h}\left(z_{n}, 2 s\right)$ imply that $\left(g \circ \varphi_{z_{n}}\right)\left(\varphi_{z_{k}}(\omega)\right)=0$ for $n \neq k$. Consequently

$$
f\left(\varphi_{z_{k}}(\omega)\right)=\left(g \circ \varphi_{z_{k}}\right)\left(\varphi_{z_{k}}(\omega)\right)=g(\omega)=\omega .
$$

Thus, if $\left(z_{\alpha}\right)$ is a net of points in $\left\{z_{n}\right\}$ that tends to $x$ then $\left(f \circ \varphi_{z_{\alpha}}\right)(\omega)=\omega$ for every $\alpha$ and every $\omega \in r \mathbb{D}$. Therefore $\left(f \circ \varphi_{x}\right)(\omega)=\omega$ when $\omega \in r \mathbb{D}$.

Suppose that (iii) fails. This means that there are $f \in \mathcal{A}, 0<r<1$ and $\varepsilon>0$ such that $\left|\left(f \circ \varphi_{z_{\alpha}}\right)\left(\xi_{\alpha}\right)-\left(f \circ \varphi_{x}\right)\left(\xi_{\alpha}\right)\right|>\varepsilon$ for some points $\xi_{\alpha} \in r \mathbb{D}$.

We can also assume that $\xi_{\alpha} \rightarrow \xi$. Since $\left(f \circ \varphi_{z_{\alpha}}\right)(\xi) \rightarrow\left(f \circ \varphi_{x}\right)(\xi)$, this contradicts the uniform $\rho$-continuity of $f$.

\subsection{The hyperbolic parts}

Definition. If $x, y \in M(\mathcal{A})$ define $\rho(x, y)=\sup \rho(\mathcal{S}, \mathcal{T})$, where $\mathcal{S}$ and $\mathcal{T}$ run over all the separated sequences in $\mathbb{D}$ so that $x \in \overline{\mathcal{S}}$ and $y \in \overline{\mathcal{T}}$. Defining $h(x, y)$ in analogous fashion, we have

$$
h(x, y)=\log \frac{1-\rho(x, y)}{1+\rho(x, y)} .
$$

Lemma 3.5 Let $x, y \in M(\mathcal{A}) \backslash \mathbb{D}$. Then

(1) $\rho(x, y)=a<1$ if and only if $y=\varphi_{x}(\omega)$ for some $\omega$ with $|\omega|=a$.

(2) $y=\varphi_{x}(\xi)$ with $\xi \in \mathbb{D}$ if and only if every separated sequences $\mathcal{S}, \mathcal{T}$ such that $x \in \overline{\mathcal{S}}$ and $y \in \overline{\mathcal{T}}$ satisfy $\rho\left(\mathcal{T},\left\{\varphi_{z_{n}}(\xi): z_{n} \in \mathcal{S}\right\}\right)=0$.

(3) $h\left(\varphi_{x}\left(\xi_{1}\right), \varphi_{x}\left(\xi_{2}\right)\right)=h\left(\xi_{1}, \xi_{2}\right)$ for every $\xi_{1}, \xi_{2} \in \mathbb{D}$.

(4) $h$ is a $[0,+\infty]$-valued metric on $M(\mathcal{A})$.

Proof. (1). Suppose that $\rho(x, y)=a<1$ and take $b \in(a, 1)$. The continuity of $\varphi_{x}$ implies that $\varphi_{x}(\overline{b \mathbb{D}})$ is compact. So, if $y \notin \varphi_{x}(\overline{b \mathbb{D}})$ there are closed disjoint neighborhoods $U$ of $\varphi_{x}(\overline{b \mathbb{D}})$ and $V$ of $y$. Let $\mathcal{S}$ and $\mathcal{T}$ be separated sequences in $\mathbb{D}$ such that $x \in \overline{\mathcal{S}}$ and $y \in \bar{T}$. If $\left(z_{\alpha}\right)$ is a net in $\mathcal{S}$ that tends to $x$ then $\varphi_{z_{\alpha}}(\xi) \rightarrow \varphi_{x}(\xi)$ for every $\xi \in \overline{b \mathbb{D}}$. By a compactness argument $\varphi_{z_{\alpha}}(\overline{b \mathbb{D}}) \subset U$ for a tail $\left(z_{\alpha}\right)_{\alpha \geq \alpha_{0}}$ of the original net. Let $\mathcal{S}_{1}=\left\{z_{n} \in \mathcal{S}: z_{n}=z_{\alpha}\right.$ for some $\left.\alpha \geq \alpha_{0}\right\}$. Then $x \in \overline{\mathcal{S}}_{1}$ and $\varphi_{z_{n}}(\overline{b \mathbb{D}}) \subset U$ for every $z_{n} \in \mathcal{S}_{1}$. This means that

$$
K\left(z_{n}, b\right) \subset U \text { for every } z_{n} \in \mathcal{S}_{1} .
$$


On the other hand, since $V$ is a neighborhood of $y$ then

$$
y \in \overline{\mathcal{T}}_{1} \text {, where } \mathcal{T}_{1}=\{z \in \mathcal{T}: z \in V\} .
$$

Since $U$ and $V$ are disjoint, (3.3) and (3.4) say that $\rho\left(\mathcal{S}_{1}, \mathcal{T}_{1}\right) \geq b>a$, contradicting the definition of $\rho(x, y)=a$. Since $b \in(a, 1)$ is arbitrary then $y \in \varphi_{x}(\overline{a \mathbb{D}})$, so $y=\varphi_{x}(\omega)$ with $|\omega| \leq a$.

Reciprocally, suppose that $y=\varphi_{x}(\omega)$ with $|\omega|=a$, and let $\mathcal{S}, \mathcal{T}$ be separated sequence in $\mathbb{D}$ such that $x \in \overline{\mathcal{S}}$ and $y \in \overline{\mathcal{T}}$. If $\left(z_{\alpha}\right)$ is a net in $\mathcal{S}$ that tends to $x$ then $\varphi_{z_{\alpha}}(\omega) \rightarrow y$. Thus $y \in \overline{\mathcal{T}}_{1}$, where $\mathcal{T}_{1}=\left\{\varphi_{z_{n}}(\omega): z_{n} \in \mathcal{S}\right\}$. So, $y \in \overline{\mathcal{T}}_{1} \cap \overline{\mathcal{T}} \neq \emptyset$ and by Lemma 3.1, $\rho\left(\mathcal{T}_{1}, \mathcal{T}\right)=0$. That is, given $\varepsilon>0$ there are $z_{n} \in \mathcal{S}$ and $\omega_{n} \in \mathcal{T}$ such that $\rho\left(\varphi_{z_{n}}(\omega), \omega_{n}\right)<\varepsilon$, which yields

$$
\rho\left(z_{n}, \omega_{n}\right) \leq \rho\left(z_{n}, \varphi_{z_{n}}(\omega)\right)+\rho\left(\varphi_{z_{n}}(\omega), \omega_{n}\right)<|\omega|+\varepsilon=a+\varepsilon .
$$

So, $\rho(\mathcal{S}, \mathcal{T}) \leq a$ and by definition $\rho(x, y) \leq a$.

(2). The necessity follows from Lemma 3.1. If $y \neq \varphi_{x}(\xi)$ then $\rho\left(y, \varphi_{x}(\xi)\right) \neq 0$ and there are separated sequences $\mathcal{T}_{1}, \mathcal{T}_{2}$ such that $\varphi_{x}(\xi) \in \overline{\mathcal{T}}_{1}, y \in \overline{\mathcal{T}}_{2}$ and $\rho\left(\mathcal{T}_{1}, \mathcal{T}_{2}\right) \geq \delta>0$. Let $\mathcal{S}$ be a separated sequence such that $x \in \overline{\mathcal{S}}$. Therefore $x$ is in the closure of $\mathcal{S}_{1}=\left\{z_{n}: \rho\left(\varphi_{z_{n}}(\xi), \mathcal{T}_{1}\right)<\delta / 2\right\}$, because if $x \in \overline{\mathcal{S} \backslash \mathcal{S}_{1}}$ then

$$
\varphi_{x}(\xi) \in \overline{\left\{\varphi_{z_{n}}(\xi): z_{n} \in \mathcal{S} \backslash \mathcal{S}_{1}\right\}} \cap \overline{\mathcal{T}}_{1}
$$

while

$$
\rho\left(\left\{\varphi_{z_{n}}(\xi): z_{n} \in \mathcal{S} \backslash \mathcal{S}_{1}\right\}, \mathcal{T}_{1}\right) \geq \delta / 2,
$$

which contradicts Lemma 3.1. So, for $z_{n} \in \mathcal{S}_{1}, \rho\left(\varphi_{z_{n}}(\xi), \mathcal{T}_{2}\right) \geq \delta / 2$.

(3). Fix $\xi_{1}, \xi_{2} \in \mathbb{D}$. By Lemma 3.2 there is a separated sequence $\mathcal{S}=\left\{z_{k}\right\}$ such that $x \in \overline{\mathcal{S}}$ and $h\left(z_{n}, z_{m}\right) \geq h\left(\xi_{1}, \xi_{2}\right)+h\left(0, \xi_{1}\right)+h\left(0, \xi_{2}\right)$ if $n \neq m$. Since

$$
\begin{aligned}
h\left(z_{n}, z_{m}\right) & \leq h\left(z_{n}, \varphi_{z_{n}}\left(\xi_{1}\right)\right)+h\left(\varphi_{z_{n}}\left(\xi_{1}\right), \varphi_{z_{m}}\left(\xi_{2}\right)\right)+h\left(\varphi_{z_{m}}\left(\xi_{2}\right), z_{m}\right) \\
& =h\left(0, \xi_{1}\right)+h\left(0, \xi_{2}\right)+h\left(\varphi_{z_{n}}\left(\xi_{1}\right), \varphi_{z_{m}}\left(\xi_{2}\right)\right),
\end{aligned}
$$

then $h\left(\varphi_{z_{n}}\left(\xi_{1}\right), \varphi_{z_{m}}\left(\xi_{2}\right)\right) \geq h\left(\xi_{1}, \xi_{2}\right)$ if $n \neq m$. Therefore

$$
h\left(\left\{\varphi_{z_{n}}\left(\xi_{1}\right)\right\}_{n \geq 1},\left\{\varphi_{z_{m}}\left(\xi_{2}\right)\right\}_{m \geq 1}\right)=h\left(\varphi_{z_{n}}\left(\xi_{1}\right), \varphi_{z_{n}}\left(\xi_{2}\right)\right)=h\left(\xi_{1}, \xi_{2}\right),
$$

implying that $h\left(\varphi_{x}\left(\xi_{1}\right), \varphi_{x}\left(\xi_{2}\right)\right) \geq h\left(\xi_{1}, \xi_{2}\right)$. For the other inequality let $\mathcal{T}_{1}, \mathcal{T}_{2}$ be separated sequences such that $\varphi_{x}\left(\xi_{1}\right) \in \overline{\mathcal{T}}_{1}$ and $\varphi_{x}\left(\xi_{2}\right) \in \overline{\mathcal{T}}_{2}$. For a separated sequence $\mathcal{S}$ such that $x \in \overline{\mathcal{S}}$ and $\varepsilon>0$ write

$$
\mathcal{S}^{\prime}=\left\{z_{n} \in \mathcal{S}: h\left(\varphi_{z_{n}}\left(\xi_{1}\right), \mathcal{T}_{1}\right)<\varepsilon, h\left(\varphi_{z_{n}}\left(\xi_{2}\right), \mathcal{T}_{2}\right)<\varepsilon\right\}
$$

and $\mathcal{S}^{\prime \prime}=\mathcal{S} \backslash \mathcal{S}^{\prime}$ 
By (2) $x \notin \overline{\mathcal{S}^{\prime \prime}}$. So, $x \in \overline{\mathcal{S}^{\prime}}$ and

$$
h\left(\mathcal{T}_{1}, \mathcal{T}_{2}\right) \leq h\left(\varphi_{z_{n}}\left(\xi_{1}\right), \varphi_{z_{n}}\left(\xi_{2}\right)\right)+2 \varepsilon=h\left(\xi_{1}, \xi_{2}\right)+2 \varepsilon .
$$

That is, $h\left(\varphi_{x}\left(\xi_{1}\right), \varphi_{x}\left(\xi_{2}\right)\right) \leq h\left(\xi_{1}, \xi_{2}\right)+2 \varepsilon$. (4). We must prove only that given $x, y, z \in M(\mathcal{A})$,

$$
h(x, y) \leq h(x, z)+h(z, y)
$$

The inequality is obvious if its right member is infinite. Otherwise (1) says that $x=\varphi_{z}\left(\xi_{1}\right)$ and $y=\varphi_{z}\left(\xi_{2}\right)$ for some $\xi_{1}, \xi_{2} \in \mathbb{D}$. Then (3.5) becomes

$$
h\left(\varphi_{z}\left(\xi_{1}\right), \varphi_{z}\left(\xi_{2}\right)\right) \leq h\left(\varphi_{z}\left(\xi_{1}\right), \varphi_{z}(0)\right)+h\left(\varphi_{z}(0), \varphi_{z}\left(\xi_{2}\right)\right),
$$

which holds by (3).

Definition. If $x \in M(\mathcal{A})$ define the hyperbolic part of $x$ as

$$
H(x)=\left\{\varphi_{x}(\omega): \omega \in \mathbb{D}\right\} .
$$

Observe that (1) of Lemma 3.5 implies that

$$
H(x)=\{y \in M(\mathcal{A}): \rho(x, y)<1\}=\{y \in M(\mathcal{A}): h(x, y)<\infty\}
$$

and by (4) of the same lemma, $\{H(x): x \in M(\mathcal{A})\}$ is a partition of $M(\mathcal{A})$. In fact if $z \in H(x) \cap H(y)$ then for any $u \in H(x)$,

$$
h(u, y) \leq h(u, x)+h(x, z)+h(z, y)<\infty .
$$

So, $H(x) \subset H(y)$ and by symmetry they coincide.

Lemma 3.6 The map $x \mapsto \varphi_{x}$ from $M(\mathcal{A})$ into $M(\mathcal{A})^{\mathbb{D}}$ is continuous.

Proof. Let $\left(x_{\alpha}\right)$ be a net in $M(\mathcal{A})$ that tends to $x$ and $\xi \in \mathbb{D}$. We must show that if $\left(x_{\beta}\right)$ is a subnet such that $\varphi_{x_{\beta}}(\xi) \rightarrow y$ then $y=\varphi_{x}(\xi)$. Let $\mathcal{S}=\left\{z_{n}\right\}$ and $\mathcal{T}=\left\{\omega_{n}\right\}$ be separated sequences such that $x \in \overline{\mathcal{S}}$ and $y \in \overline{\mathcal{T}}$. For $\delta>0$ write

$$
U=\bigcup_{n \geq 1} K\left(z_{n}, \delta\right) \text { and } V=\bigcup_{n \geq 1} K\left(\omega_{n}, \delta\right) .
$$

Since there is $f \in \mathcal{A}$ such that $f\left(z_{n}\right)=0$ for all $n$ and $f \equiv 1$ on $\mathbb{D} \backslash U$ then $\bar{U} \supset\{m \in M(\mathcal{A}):|f(m)|<1 / 2\}$, a neighborhood of $x$. So, $\bar{U}$ is a neighborhood of $x$ and by the same reason $\bar{V}$ is a neighborhood of $y$. Since $x_{\beta} \rightarrow x$ and $\varphi_{x_{\beta}}(\xi) \rightarrow y$, there is $\beta_{0}$ such that for every $\beta \geq \beta_{0}$,

(i) $\varphi_{x_{\beta}}(\xi) \in \bar{V}$, and

(ii) $x_{\beta} \in \overline{\mathcal{S}}_{\beta}$, where $\mathcal{S}_{\beta}=\left\{z_{n}(\beta)\right\}_{n \geq 1}$ is a separated sequence in $U$. 
Assume that $\beta \geq \beta_{0}$. Since

$$
\varphi_{x_{\beta}}(\xi) \in{\overline{\left\{\varphi_{z_{n}(\beta)}(\xi)\right\}_{n \geq 1}}}_{n\left(\overline{\cup_{n} K\left(\omega_{n}, \delta\right)}\right)}
$$

then Lemma 3.1 says that $\rho\left(\left\{\varphi_{z_{n}(\beta)}(\xi)\right\}, \mathcal{T}\right) \leq \delta$. So, there is $n_{0}$ such that $\rho\left(\varphi_{z_{n_{0}}(\beta)}(\xi), \mathcal{T}\right)<2 \delta$. On the other hand, by definition of $U$ and (ii) there is some $z_{k_{0}} \in \mathcal{S}$ such that $\rho\left(z_{k_{0}}, z_{n_{0}}(\beta)\right) \leq \delta$. Since there is $c_{\xi}>0$ such that

$$
\rho\left(\varphi_{z_{k_{0}}}(\xi), \varphi_{z_{n_{0}}(\beta)}(\xi)\right) \leq c_{\xi} \rho\left(z_{k_{0}}, z_{n_{0}}(\beta)\right) \leq c_{\xi} \delta
$$

then $\rho\left(\varphi_{z_{k_{0}}}(\xi), \mathcal{T}\right) \leq\left(c_{\xi}+2\right) \delta$. This shows that

$$
\rho\left(\left\{\varphi_{z_{n}}(\xi): z_{n} \in \mathcal{S}\right\}, \mathcal{T}\right) \leq\left(c_{\xi}+2\right) \delta,
$$

and since $\delta>0$ is arbitrary, $\rho\left(\left\{\varphi_{z_{n}}(\xi): z_{n} \in \mathcal{S}\right\}, \mathcal{T}\right)=0$. Since $\mathcal{S}$ and $\mathcal{T}$ are arbitrary separated sequences such that $x \in \overline{\mathcal{S}}$ and $y \in \overline{\mathcal{T}}$ then $(2)$ of Lemma 3.5 tells us that $y=\varphi_{x}(\xi)$.

\subsection{Hyperbolic algebras}

A closed self-adjoint subalgebra $\mathcal{B}$ of $\mathcal{A}$ that separates the points of $\mathbb{D}$ and contains the constants will be called a prehyperbolic algebra. For such $\mathcal{B}$, Theorem 4.28 of [13] implies that whenever $b \in \mathcal{B}$ is invertible in $\mathcal{A}$ then the inverse belongs to $\mathcal{B}$. Hence, the disk is dense in $M(\mathcal{B})$, because if there exists $y \in M(\mathcal{B})$ that is not in the closure of $\mathbb{D}$ then there is $f \in \mathcal{B}$ such that $f(y)=0$ and $|f| \geq \delta>0$ on $\mathbb{D}$. Since clearly $f$ is invertible in $\mathcal{A}$, then so is in $\mathcal{B}$ and consequently $f$ cannot vanish anywhere in $M(\mathcal{B})$, a contradiction.

The inclusion of $\mathcal{B}$ in $\mathcal{A}$ induces by transposition a projection $\pi: M(\mathcal{A}) \rightarrow$ $M(\mathcal{B})$. Since $\pi(\mathbb{D})=\mathbb{D}$ is dense in $M(\mathcal{B})$ then $\pi$ is onto. For a set $E \subset \mathbb{D}$ we write $\bar{E}^{M}$, with $M=M(\mathcal{A})$ or $M(\mathcal{B})$, to distinguish between closures in the corresponding space. No distinction will be made for the closure of sets in $\mathbb{C}$.

A closed set $F \subset M(\mathcal{A})$ will be called saturated if $H(x) \subset F$ whenever $x \in F$. If $\pi: M(\mathcal{A}) \rightarrow M(\mathcal{B})$ is the natural projection, write

$$
G_{\mathcal{B}}=\left\{y \in M(\mathcal{B}): \pi^{-1}(y) \text { is a singleton }\right\}
$$

and

$$
\Gamma_{\mathcal{B}}=\left\{y \in M(\mathcal{B}): \pi^{-1}(y) \text { is saturated }\right\} .
$$

That is, if $y \in M(\mathcal{B})$ then $y \in G_{\mathcal{B}}$ if and only if $\mathcal{B}$ separates every $x \in \pi^{-1}(y)$ from any other point of $M(\mathcal{A})$ (so $\pi^{-1}(y)=\{x\}$ ), and $y \in \Gamma_{\mathcal{B}}$ if and only if $b \circ \varphi_{x}$ is constant for all $x \in \pi^{-1}(y)$ and $b \in \mathcal{B}$. Since no single point is a saturated set then $G_{\mathcal{B}} \cap \Gamma_{\mathcal{B}}=\emptyset$. In addition, there could be points in $M(\mathcal{B})$ that are not in $G_{\mathcal{B}} \cup \Gamma_{\mathcal{B}}$. We will be interested in the cases that exclude the last possibility. 
Definition. A prehyperbolic algebra $\mathcal{B}$ will be called hyperbolic if $M(\mathcal{B})=$ $G_{\mathcal{B}} \cup \Gamma_{\mathcal{B}}$. That is, if $\pi^{-1}(\pi(x))=\{x\}$ or contains $H(x)$ for every $x \in M(\mathcal{A})$.

Lemma 3.7 Let $\mathcal{B} \subset \mathcal{A}$ be a prehyperbolic algebra. Then

(1) $\Gamma_{\mathcal{B}}$ is closed,

(2) the restriction $\pi_{0}: \pi^{-1}\left(G_{\mathcal{B}}\right) \rightarrow G_{\mathcal{B}}$ of $\pi$ is an onto homeomorphism.

Proof. (1). If $x$ is in the closure of $\pi^{-1}\left(\Gamma_{\mathcal{B}}\right)$ take a net $\left(x_{\alpha}\right)$ in $\pi^{-1}\left(\Gamma_{\mathcal{B}}\right)$ that tends to $x$. By definition of $\Gamma_{\mathcal{B}}, f \circ \varphi_{x_{\alpha}}$ is constant for every $f \in \mathcal{B}$. Hence, if $\omega \in \mathbb{D}$ and $f \in \mathcal{B}$, Lemma 3.6 gives

$$
f(x)-f\left(\varphi_{x}(\omega)\right)=\lim f\left(x_{\alpha}\right)-f\left(\varphi_{x_{\alpha}}(\omega)\right)=0,
$$

implying that $f \circ \varphi_{x} \equiv f(x)$, so $x \in \pi^{-1}\left(\Gamma_{\mathcal{B}}\right)$. That is, $\pi^{-1}\left(\Gamma_{\mathcal{B}}\right)$ is closed in $M(\mathcal{A})$ and then $\pi\left(\pi^{-1}\left(\Gamma_{\mathcal{B}}\right)\right)$ is closed in $M(\mathcal{B})$.

(2). By definition of $G_{\mathcal{B}}, \pi_{0}$ is one-to-one and onto, so we must show that $\pi_{0}^{-1}: G_{\mathcal{B}} \rightarrow \pi^{-1}\left(G_{\mathcal{B}}\right)$ is continuous. Let $\left(y_{\alpha}\right)$ be a net in $G_{\mathcal{B}}$ such that $y_{\alpha} \rightarrow y \in G_{\mathcal{B}}$ and let $x_{\alpha} \in \pi^{-1}\left(G_{\mathcal{B}}\right)$ such that $\pi\left(x_{\alpha}\right)=y_{\alpha}$. If $\left(x_{\alpha_{\beta}}\right)$ is a convergent subnet of $\left(x_{\alpha}\right)$, say to $x$, then $y_{\alpha_{\beta}}=\pi\left(x_{\alpha_{\beta}}\right) \rightarrow \pi(x)=y$. So, $x \in \pi^{-1}(y)$, but since $y \in G_{\mathcal{B}}$ then $\pi^{-1}(y)=\{x\}$. Hence every convergent subnet of $\left(x_{\alpha}\right)$ tends to $x$, and then $x_{\alpha} \rightarrow x$.

Proposition 3.8 Let $\mathcal{B} \subset \mathcal{A}$ be a prehyperbolic algebra and $y \in M(\mathcal{B})$. The following conditions are equivalent

$\left(\mathrm{a}_{1}\right) y \in \Gamma_{\mathcal{B}}$.

$\left(\mathrm{a}_{2}\right) f \circ \varphi_{z_{\alpha}} \rightarrow c \in \mathbb{C}$ uniformly on compact sets for every net $\left(z_{\alpha}\right)$ in $\mathbb{D}$ tending to $y$ and every $f \in \mathcal{B}$.

$\left(\mathrm{a}_{3}\right)$ For every separated sequence $\mathcal{S}$ such that $y \in \overline{\mathcal{S}}^{M(\mathcal{B})}$ and every $f \in \mathcal{B}$ there is a subsequence $\left\{z_{n}\right\}$ of $\mathcal{S}$ (depending on $f$ ) such that $f \circ \varphi_{z_{n}} \rightarrow c \in$ $\mathbb{C}$ pointwise on $\mathbb{D}$.

Proof. $\quad\left(\mathrm{a}_{1}\right) \Rightarrow\left(\mathrm{a}_{2}\right)$. If $y \in \Gamma_{\mathcal{B}}$ then $\pi^{-1}(y)$ is saturated. Let $\left(z_{\alpha}\right)$ be a net in $\mathbb{D}$ such that $z_{\alpha} \rightarrow y$ in $M(\mathcal{B})$ and take a subnet $\left(z_{\alpha_{\beta}}\right)$ that converges in $M(\mathcal{A})$, say to $x$. Thus $\pi\left(z_{\alpha_{\beta}}\right) \rightarrow \pi(x)=y$ in $M(\mathcal{B})$, saying that $x \in \pi^{-1}(y)$. Since $H(x) \subset \pi^{-1}(y)$ (because it is saturated) then

$$
f\left(\varphi_{x}(\xi)\right)=\lim f\left(\varphi_{z_{\alpha_{\beta}}}(\xi)\right)=\text { const. }=\lim f\left(\varphi_{z_{\alpha_{\beta}}}(0)\right)=\lim f\left(z_{\alpha_{\beta}}\right)=f(y)
$$

for every $f \in \mathcal{B}$ and $\xi \in \mathbb{D}$. This proves that whenever $\left(z_{\alpha_{\beta}}\right)$ is a subnet of $\left(z_{\alpha}\right)$ that converges in $M(\mathcal{A})$ then $f \circ \varphi_{z_{\alpha_{\beta}}} \rightarrow f(y)$ pointwise. By Lemma 3.4 the convergence is also uniform on compact sets, and consequently $f \circ \varphi_{z_{\alpha}} \rightarrow f(y)$ in that way. 
$\left(\mathrm{a}_{2}\right) \Rightarrow\left(\mathrm{a}_{3}\right)$. If $y \in \overline{\mathcal{S}}^{M(\mathcal{B})}$ there is a net $\left(z_{\alpha}\right)$ in $\mathcal{S}$ such that $z_{\alpha} \rightarrow y$ in $M(\mathcal{B})$. If $f \in \mathcal{B}$ then by $\left(\mathrm{a}_{2}\right), f \circ \varphi_{z_{\alpha}} \rightarrow c \in \mathbb{C}$ uniformly on compact sets. Therefore for any positive integer $n$ there is some $z_{\alpha}$ (that we rename as $z_{n}$ ) such that

$$
\sup \left\{\left|\left(f \circ \varphi_{z_{n}}\right)(\omega)-c\right|:|\omega| \leq 1-n^{-1}\right\} \leq n^{-1} .
$$

Therefore $\left\{z_{n}\right\}$ is a subsequence of $\mathcal{S}$ that satisfies $\left(\mathrm{a}_{3}\right)$.

$\left(\mathrm{a}_{3}\right) \Rightarrow\left(\mathrm{a}_{1}\right)$. We will show that $\left(\mathrm{a}_{3}\right)$ fails when $\left(\mathrm{a}_{1}\right)$ fails. If $y \notin \Gamma_{\mathcal{B}}$ there is $x \in \pi^{-1}(y)$ such that $H(x) \not \subset \pi^{-1}(y)$. Therefore there is $f \in \mathcal{B}$ such that $f \circ \varphi_{x} \neq$ const., or what is the same, $\left(f \circ \varphi_{x}\right)(\omega) \neq f(x)$ for some $\omega \in \mathbb{D}$. Put $\eta=\left|\left(f \circ \varphi_{x}\right)(\omega)-f(x)\right|>0$. If $\mathcal{S}$ is any separated sequence such that $x \in \overline{\mathcal{S}}^{M(\mathcal{A})}$ and we take

$$
\mathcal{S}_{1}=\left\{z \in \mathcal{S}:\left|\left(f \circ \varphi_{z_{n}}\right)(\omega)-f\left(z_{n}\right)\right| \geq \eta / 2\right\}
$$

then $x \in \overline{\mathcal{S}}_{1}^{M(\mathcal{A})}$. Hence $y=\pi(x) \in \overline{\mathcal{S}}_{1}^{M(\mathcal{B})}$ and $\left(\mathrm{a}_{3}\right)$ fails for $\mathcal{S}_{1}$ and $f$.

Suppose that $f$ is a continuous function from $M(\mathcal{A})$ into a topological space $T$. If $\mathcal{B}$ is a hyperbolic algebra, the restriction $\left.f\right|_{\mathbb{D}}$ admits a continuous extension from $M(\mathcal{B})$ into $T$ if and only if $f\left(\pi^{-1}(y)\right)=$ const. for every $y \in \Gamma_{\mathcal{B}}$. In particular, for $T=\mathbb{C}$ we obtain that $f \in \mathcal{A}$ belongs to $\mathcal{B}$ if and only if $f\left(\pi^{-1}(y)\right)=$ const. for every $y \in \Gamma_{\mathcal{B}}$.

Let $\mathcal{B} \subset L^{\infty}(\mathbb{D})$ be a closed algebra. A sequence $\left\{z_{n}\right\} \subset \mathbb{D}$ is called interpolating for $\mathcal{B}$ if for every $\left\{\eta_{n}\right\} \in \ell^{\infty}$ there exists $f \in \mathcal{B}$ such that $f\left(z_{n}\right)=\eta_{n}$ for every $n$. It is clear that if $\mathcal{B}$ is a subalgebra of $\mathcal{A}$ then every interpolating sequence for $\mathcal{B}$ must be separated and that every separated sequence is interpolating for $\mathcal{A}$. We say that $f \in \mathcal{A}$ separates two sets $E, F \subset M(\mathcal{A})$ when $\overline{f(E)} \cap \overline{f(F)}=\emptyset$.

Proposition 3.9 Let $\mathcal{B} \subset \mathcal{A}$ be a prehyperbolic algebra. For $y \in M(\mathcal{B})$ consider the following conditions

$\left(\mathrm{b}_{1}\right) y \in G_{\mathcal{B}}$.

$\left(\mathrm{b}_{2}\right)$ There is an interpolating sequence $\mathcal{S}=\left\{z_{n}\right\}$ for $\mathcal{B}$, whose closure in $M(\mathcal{B})$ contains $y$, such that for every $\delta>0$ sufficiently small there exists $f \in \mathcal{B}$ that separates $\left\{z_{n}\right\}$ from $\mathbb{D} \backslash \bigcup_{n} K\left(z_{n}, \delta\right)$.

Then $\left(\mathrm{b}_{2}\right)$ implies $\left(\mathrm{b}_{1}\right)$, and if $\mathcal{B}$ is hyperbolic, $\left(\mathrm{b}_{1}\right)$ implies $\left(\mathrm{b}_{2}\right)$.

Proof. $\quad\left(\mathrm{b}_{2}\right) \Rightarrow\left(\mathrm{b}_{1}\right)$. Let $y \in M(\mathcal{B})$ and $\mathcal{S}$ as in $\left(\mathrm{b}_{2}\right)$. We claim that $\pi^{-1}(y) \subset \overline{\mathcal{S}}^{M(\mathcal{A})}$, because otherwise there is $x \in \pi^{-1}(y)$ and a separated sequence $\mathcal{T} \subset \mathbb{D}$, with $x \in \overline{\mathcal{T}}^{M(\mathcal{A})}$, such that $\rho(\mathcal{S}, \mathcal{T}) \geq \alpha>0$. The continuity of $\pi$ implies that $y=\pi(x) \in \overline{\mathcal{T}}^{M(\mathcal{B})}$, but this is not possible because by hypothesis there is $f \in \mathcal{B}$ such that $\overline{f(\mathcal{S})} \cap \overline{f(\mathcal{T})}=\emptyset$, which contradicts $y \in \overline{\mathcal{S}}^{M(\mathcal{B})} \cap \overline{\mathcal{T}}^{M(\mathcal{B})}$. 
Now suppose that there are two different points $x_{1}, x_{2} \in \pi^{-1}(y)$. Then there is a disjoint decomposition $\mathcal{S}=\mathcal{S}_{1} \cup \mathcal{S}_{2}$, where

$$
x_{1} \in \overline{\mathcal{S}}_{1}^{M(\mathcal{A})} \quad \text { and } \quad x_{2} \in \overline{\mathcal{S}}_{2}^{M(\mathcal{A})} .
$$

Since $\mathcal{S}$ is interpolating for $\mathcal{B}$ there exists $f \in \mathcal{B}$ that separates $\mathcal{S}_{1}$ from $\mathcal{S}_{2}$, leading to the same contradiction obtained before. Hence, $\pi^{-1}(y)$ is a single point.

$\left(\mathrm{b}_{1}\right) \Rightarrow\left(\mathrm{b}_{2}\right)$ for $\mathcal{B}$ hyperbolic. If $y \in G_{\mathcal{B}}$ then $\pi^{-1}(y)=\{x\}$ for some $x \in M(\mathcal{A})$. Since $\pi^{-1}\left(\Gamma_{\mathcal{B}}\right)$ is closed in $M(\mathcal{A})$ (by Lemma 3.7) and $x \notin$ $\pi^{-1}\left(\Gamma_{\mathcal{B}}\right)$ then there is a closed neighborhood $F$ of $x$ in $M(\mathcal{A})$ such that $F \cap \pi^{-1}\left(\Gamma_{\mathcal{B}}\right)=\emptyset$. Hence there is $f \in \mathcal{A}$ such that $f \equiv 1$ on $F$ and $f \equiv 0$ on $\pi^{-1}\left(\Gamma_{\mathcal{B}}\right)$.

Let $\mathcal{T} \subset \mathbb{D}$ be a separated sequence such that $x \in \overline{\mathcal{T}}^{M(\mathcal{A})}$. Since $f \equiv 1$ on a neighborhood of $x$ then $x \in \overline{\mathcal{S}}^{M(\mathcal{A})}$, where

$$
\mathcal{S}=\{z \in \mathcal{T}: f(z)=1\}=\left\{z_{n}\right\} .
$$

Hence, $y=\pi(x) \in \overline{\mathcal{S}}^{M(\mathcal{B})}$. Observe also that $\overline{\mathcal{S}}^{M(\mathcal{A})} \subset F \subset \pi^{-1}\left(G_{\mathcal{B}}\right)$.

Let $\left\{\eta_{n}\right\}$ be an arbitrary sequence in $\ell^{\infty}$ and take $g \in \mathcal{A}$ such that $g\left(z_{n}\right)=\eta_{n}$ for every $n$. Since $f \equiv 0$ on $\pi^{-1}\left(\Gamma_{\mathcal{B}}\right)$ then so is $h=f g \in \mathcal{A}$, and consequently $h \in \mathcal{B}$. In addition, $h\left(z_{n}\right)=f\left(z_{n}\right) g\left(z_{n}\right)=\eta_{n}$ for every $n$, which shows that $\mathcal{S}$ is interpolating for $\mathcal{B}$. Since $f$ is $\rho$-uniformly continuous and $f\left(z_{n}\right)=1$ for all $n$ then

$$
\bigcup K\left(z_{n}, \delta\right) \subset\{z:|f(z)|>1 / 2\}
$$

when $\delta>0$ is small enough. Take $a \in \mathcal{A}$ such that

$$
a\left(z_{n}\right)=1 \text { for all } n \text {, and } a \equiv 0 \text { on } \mathbb{D} \backslash \bigcup_{n} K\left(z_{n}, \delta\right) \text {. }
$$

Since $f \equiv 0$ on $\pi^{-1}\left(\Gamma_{\mathcal{B}}\right)$ then

$$
\pi^{-1}\left(\Gamma_{\mathcal{B}}\right) \subset \overline{\{z:|f(z)|<1 / 4\}}^{M(\mathcal{A})} \subset{\overline{\mathbb{D} \backslash \bigcup_{n} K\left(z_{n}, \delta\right)}}^{M(\mathcal{A})},
$$

implying that $a \equiv 0$ on $\pi^{-1}\left(\Gamma_{\mathcal{B}}\right)$. Hence $a \in \mathcal{B}$ and (3.6) says that it separates $\mathcal{S}$ from $\mathbb{D} \backslash \bigcup_{n} K\left(z_{n}, \delta\right)$. So $\left(\mathrm{b}_{2}\right)$ holds.

Propositions 3.8 and 3.9 provide criteria to decide whether a given prehyperbolic algebra is hyperbolic or not. Let us summarize these criteria in the next corollary.

Corollary 3.10 A prehyperbolic algebra $\mathcal{B}$ is hyperbolic if and only if every $y \in M(\mathcal{B})$ satisfies some of the conditions $\left(\mathrm{a}_{1}\right),\left(\mathrm{a}_{2}\right),\left(\mathrm{a}_{3}\right)$ or some of the conditions $\left(\mathrm{b}_{1}\right),\left(\mathrm{b}_{2}\right)$. 


\section{Operator-valued compact maps}

We recall that if $S \in \mathfrak{L}\left(L_{a}^{2}\right)$ and $z \in \mathbb{D}$ then $S_{z}=U_{z} S U_{z}$, where $U_{z} f=$ $\left(f \circ \varphi_{z}\right) \varphi_{z}^{\prime}$. Consider the map $\Psi_{S}: \mathbb{D} \rightarrow \mathfrak{L}\left(L_{a}^{2}\right)$ given by $\Psi_{S}(z)=S_{z}$. We will study the possibility to extend $\Psi_{S}$ continuously to $M(\mathcal{A})$ when $\mathfrak{L}\left(L_{a}^{2}\right)$ is provided with the weak or the strong operator topology (WOT and SOT, respectively). We will also look for a possible extension to $M(\mathcal{B})$, where $\mathcal{B}$ is an arbitrary hyperbolic algebra.

Theorem 4.1 Let $(E, d)$ be a metric space and $f: \mathbb{D} \rightarrow E$ be a continuous map. Then $f$ admits a continuous extension from $M(\mathcal{A})$ into $E$ if and only if $f$ is uniformly $(\rho, d)$ continuous and $\overline{f(\mathbb{D})}$ is compact.

Proof. Suppose that $f \in C(M(\mathcal{A}), E)$. Since $\mathbb{D}$ is dense in the compact space $M(\mathcal{A})$ then $\overline{f(\mathbb{D})}=f(M(\mathcal{A}))$ is compact. If $f$ is not uniformly $(\rho, d)$ continuous there are two sequences $z_{n}, \omega_{n} \in \mathbb{D}$ such that $\rho\left(z_{n}, \omega_{n}\right) \rightarrow 0$ and $d\left(f\left(z_{n}\right), f\left(\omega_{n}\right)\right) \geq \delta>0$ for every $n$. By the continuity of $f$ on $\mathbb{D}$ the sequence

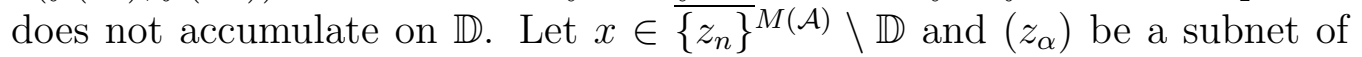
$\left\{z_{n}\right\}$ that tends to $x$. Since every $z_{\alpha}$ is some $z_{n(\alpha)}$, writing $\omega_{\alpha}=\omega_{n(\alpha)}$ we have a subnet $\left(\omega_{\alpha}\right)$ of the sequence $\left\{\omega_{n}\right\}$ such that

$$
\rho\left(z_{\alpha}, \omega_{\alpha}\right) \rightarrow 0 \text { and } d\left(f\left(z_{\alpha}\right), f\left(\omega_{\alpha}\right)\right) \geq \delta \text { for all } \alpha .
$$

The first condition in (4.1) implies that $g\left(\omega_{\alpha}\right) \rightarrow g(x)$ for every $g \in \mathcal{A}$, meaning that $\omega_{\alpha} \rightarrow x$ in $M(\mathcal{A})$. Since $f$ is continuous on $M(\mathcal{A})$ then $\lim f\left(\omega_{\alpha}\right)=$ $f(x)=\lim f\left(z_{\alpha}\right)$, contradicting (4.1).

Now assume that $f$ is uniformly $(\rho, d)$ continuous on $\mathbb{D}$ and $\overline{f(\mathbb{D})}$ is compact. For $x \in M(\mathcal{A})$ write

$$
F(x) \stackrel{\text { def }}{=}\left\{\lambda \in E: f\left(z_{\alpha}\right) \rightarrow \lambda, \text { for some net } z_{\alpha} \rightarrow x, z_{\alpha} \in \mathbb{D}\right\} .
$$

The compactness of $\overline{f(\mathbb{D})}$ assures that $F(x)$ is nonempty. Then $F$ is a multivalued function defined on $M(\mathcal{A})$, and a standard diagonal argument shows that $f$ can be extended continuously to $M(\mathcal{A})$ if and only if $F(x)$ is single-valued for every $x \in M(\mathcal{A})$. So, let $x \in M(\mathcal{A})$ and assume that there are $\lambda_{1}, \lambda_{2} \in F(x)$ such that $d\left(\lambda_{1}, \lambda_{2}\right)=\alpha>0$. Let $B(\lambda, r)$ denote the open ball in $E$ of center $\lambda \in E$ and radius $r>0$, and consider the sets

$$
V_{i}=\left\{z \in \mathbb{D}: f(z) \in B\left(\lambda_{i}, \alpha / 4\right)\right\}, \quad i=1,2 .
$$

Since $\lambda_{i} \in F(x)$ then $x \in \bar{V}_{i}^{M(\mathcal{A})}$ for $i=1,2$. Lemma 3.1 then tells us that $\rho\left(V_{1}, V_{2}\right)=0$. On the other hand,

$$
d\left(f\left(V_{1}\right), f\left(V_{2}\right)\right) \geq d\left(B\left(\lambda_{1}, \alpha / 4\right), B\left(\lambda_{2}, \alpha / 4\right)\right) \geq \frac{\alpha}{2} .
$$

By the uniform $(\rho, d)$-continuity of $f$, the last inequality implies that $\rho\left(V_{1}, V_{2}\right)>0$, a contradiction. 
Lemma 4.2 For $z, \alpha \in \mathbb{D}$ put $\lambda=\lambda(z, \alpha)=(\alpha \bar{z}-1) /(1-z \bar{\alpha})$. Then $U_{\varphi_{z}(\alpha)} U_{z}=V_{\lambda} U_{\alpha}$, where $\left(V_{\lambda} f\right)(\omega)=\lambda f(\lambda \omega)$ for $f \in L_{a}^{2}$.

Proof. Since the function $\varphi_{\varphi_{z}(\alpha)} \circ \varphi_{z} \circ \varphi_{\alpha}$ is an automorphism that fixes the origin, it must be a rotation. A little bit of algebra shows that this function maps $\lambda$ to 1 . Since $\varphi_{\varphi_{z}(\alpha)}$ is its own inverse then $\varphi_{z} \circ \varphi_{\alpha}(\lambda \omega)=\varphi_{\varphi_{z}(\alpha)}(\omega)$. Therefore

$$
\begin{aligned}
& \left(U_{\varphi_{z}(\alpha)} U_{z} f\right)(\omega)=\left(f \circ \varphi_{z} \circ \varphi_{\varphi_{z}(\alpha)}\right)(\omega) \varphi_{z}^{\prime}\left(\varphi_{\varphi_{z}(\alpha)}(\omega)\right) \varphi_{\varphi_{z}(\alpha)}^{\prime}(\omega) \\
& \quad=\left(f \circ \varphi_{z} \circ \varphi_{z} \circ \varphi_{\alpha}\right)(\lambda \omega) \varphi_{z}^{\prime}\left(\varphi_{z} \circ \varphi_{\alpha}(\lambda \omega)\right) \varphi_{z}^{\prime}\left(\varphi_{\alpha}(\lambda \omega)\right) \varphi_{\alpha}^{\prime}(\lambda \omega) \lambda \\
& \quad=\left(f \circ \varphi_{\alpha}\right)(\lambda \omega) \varphi_{\alpha}^{\prime}(\lambda \omega) \lambda=\left(V_{\lambda} U_{\alpha} f\right)(\omega),
\end{aligned}
$$

where the third equality holds because since $\varphi_{z} \circ \varphi_{z}=i d$ then $\left(\varphi_{z}^{\prime} \circ \varphi_{z}\right) \varphi_{z}^{\prime}=1$.

Lemma 4.3 Let $f \in L_{a}^{2}$ and $\varepsilon>0$. Then there is $\delta=\delta(f, \varepsilon)>0$ such that

$$
\rho\left(z_{1}, z_{2}\right)<\delta \Rightarrow\left\|U_{z_{1}} f-U_{z_{2}} f\right\|<\varepsilon .
$$

Proof. Since the polynomials are dense in $L_{a}^{2}$ and $\left\|U_{z}\right\|=1$ for every $z \in \mathbb{D}$, it is enough to assume that $f$ is a polynomial. If $\rho\left(z_{1}, z_{2}\right)<\delta$ then $z_{2}=\varphi_{z_{1}}(\alpha)$ with $|\alpha|<\delta$. By the previous lemma,

$$
\left(I-U_{\varphi_{z_{1}}(\alpha)} U_{z_{1}}\right) f(\omega)=f(\omega)-f\left(\frac{\alpha-\lambda \omega}{1-\bar{\alpha} \lambda \omega}\right)\left(\frac{|\alpha|^{2}-1}{1-\bar{\alpha} \lambda \omega}\right) \lambda,
$$

where $\lambda$ comes from the lemma. When $\alpha \rightarrow 0$ we have $\lambda\left(z_{1}, \alpha\right) \rightarrow-1$ uniformly in $z_{1}$, so the above expression tends to 0 uniformly in $z_{1}$ and $\omega$. Hence,

$$
\left\|U_{z_{1}} f-U_{\varphi_{z_{1}}(\alpha)} f\right\|=\left\|\left(U_{\varphi_{z_{1}}(\alpha)} U_{z_{1}}-I\right) f\right\|<\varepsilon
$$

if $|\alpha|$ is small enough. That is, if $\delta$ is small enough.

Proposition 4.4 Let $S \in \mathfrak{L}\left(L_{a}^{2}\right)$. Then the map $\Psi_{S}: \mathbb{D} \rightarrow\left(\mathfrak{L}\left(L_{a}^{2}\right)\right.$, WOT $)$ extends continuously to $M(\mathcal{A})$.

Proof. The closed the ball $B(0,\|S\|) \subset \mathfrak{L}\left(L_{a}^{2}\right)$ of center 0 and radius $\|S\|$ is compact and metrizable with the WOT-topology. Since $\Psi_{S}(\mathbb{D})$ is contained in $B(0,\|S\|)$, Theorem 4.1 reduces the problem to show that $\Psi_{S}$ is uniformly continuous from the disk with the pseudo-hyperbolic metric into $B(0,\|S\|)$ with the weak operator topology. This amounts to see that for every $f, g \in$ $L_{a}^{2}$, the function $z \mapsto\left\langle S_{z} f, g\right\rangle$ is uniformly continuous from $(\mathbb{D}, \rho)$ into $(\mathbb{C},||)$. For $z_{1}, z_{2} \in \mathbb{D}$ we have

$$
U_{z_{1}} S U_{z_{1}}-U_{z_{2}} S U_{z_{2}}=U_{z_{1}} S\left(U_{z_{1}}-U_{z_{2}}\right)+\left(U_{z_{1}}-U_{z_{2}}\right) S U_{z_{2}}=A+B .
$$

If $f, g \in L_{a}^{2}$ then $|\langle A f, g\rangle| \leq\left\|U_{z_{1}} S\right\|\left\|\left(U_{z_{1}}-U_{z_{2}}\right) f\right\|_{2}\|g\|_{2}$ and $|\langle B f, g\rangle|=$ $\left|\left\langle f, B^{*} g\right\rangle\right| \leq\|f\|_{2}\left\|U_{z_{2}} S^{*}\right\|\left\|\left(U_{z_{1}}-U_{z_{2}}\right) g\right\|_{2}$. By Lemma 4.3 both expressions can be made small if we take $\rho\left(z_{1}, z_{2}\right)$ small enough. 
Theorem 4.5 Let $S \in \mathfrak{T}(\mathcal{A})$. Then the map

$$
\Psi_{S}: \mathbb{D} \rightarrow\left(\mathfrak{L}\left(L_{a}^{2}\right), S O T\right)
$$

extends continuously to $M(\mathcal{A})$. In addition, $\Psi_{S}(M(\mathcal{A})) \subset \mathfrak{T}(\mathcal{A})$.

Proof. First suppose that $S=T_{a}$, with $a \in \mathcal{A}$. If $z \in \mathbb{D}$ tends to $x \in M(\mathcal{A})$, Lemma 3.4 says that $a \circ \varphi_{z} \rightarrow a \circ \varphi_{x}$ uniformly on compact sets. Thus, if $f \in L_{a}^{2}$ and $0<r<1$,

$$
\left\|\left(T_{a \circ \varphi_{z}}-T_{a \circ \varphi_{x}}\right) f\right\|^{2} \leq \sup _{r D}\left|a \circ \varphi_{z}-a \circ \varphi_{x}\right|^{2}\|f\|^{2}+2\|a\|_{\infty}^{2} \int_{D \backslash r D}|f|^{2} d A .
$$

We can choose some $r=r\left(f,\|a\|_{\infty}\right)$ close enough to 1 so that the second term is smaller than a given $\varepsilon>0$, and for such $r$ the first term tends to 0 as $z \rightarrow x$. Since

$$
\Psi_{S+T}=\Psi_{S}+\Psi_{T}
$$

the case of a polynomial in Toeplitz operators reduces to the case $S=$ $T_{a_{1}} \ldots T_{a_{k}}$, where $a_{j} \in \mathcal{A}$ and $\left\|a_{j}\right\|_{\infty} \leq 1$ for $j=1, \ldots, k$. Consider the operators

$$
S_{j}= \begin{cases}T_{a_{1} \circ \varphi_{z}} \ldots T_{a_{j-1} \circ \varphi_{z}} T_{a_{j} \circ \varphi_{x}} \ldots T_{a_{k} \circ \varphi_{x}} & \text { if } 1 \leq j \leq k \\ T_{a_{1} \circ \varphi_{z}} \ldots T_{a_{k} \circ \varphi_{z}} & \text { if } j=k+1\end{cases}
$$

If $f \in L_{a}^{2}$ then

$$
\left\|\left(S_{k+1}-S_{1}\right) f\right\| \leq \sum_{j=1}^{k}\left\|\left(S_{j+1}-S_{j}\right) f\right\|
$$

and since we have proved that $T_{a_{j} \circ \varphi_{z}}-T_{a_{j} \circ \varphi_{x}} \rightarrow 0$ in the strong operator topology as $z \rightarrow x$, then

$$
\begin{aligned}
\left\|\left(S_{j+1}-S_{j}\right) f\right\| & =\left\|T_{a_{1} \circ \varphi_{z}} \ldots T_{a_{j-1} \circ \varphi_{z}}\left(T_{a_{j} \circ \varphi_{z}}-T_{a_{j} \circ \varphi_{x}}\right) T_{a_{j+1} \circ \varphi_{x}} \ldots T_{a_{k} \circ \varphi_{x}} f\right\| \\
& \leq\left\|\left(T_{a_{j} \circ \varphi_{z}}-T_{a_{j} \circ \varphi_{x}}\right) T_{a_{j+1} \circ \varphi_{x}} \ldots T_{a_{k} \circ \varphi_{x}} f\right\| \rightarrow 0
\end{aligned}
$$

when $z \rightarrow x$. Finally, if $S \in \mathfrak{T}(\mathcal{A})$ is arbitrary, given $\varepsilon>0$ there is a polynomial in Toeplitz operators with symbols in $\mathcal{A}$, say $T$, such that $\|S-T\|<\varepsilon$. By Proposition 4.4 there is some $S_{x} \in \mathfrak{L}\left(L_{a}^{2}\right)$ (i.e. : $\left.S_{x}=\Psi_{S}(x)\right)$ such that

$$
S_{z}-T_{z} \rightarrow S_{x}-T_{x} \quad \text { weakly when } z \rightarrow x .
$$

Weak limits do not increase norms, so $\left\|S_{x}-T_{x}\right\| \leq \varepsilon$. The result follows because $\left\|S_{z}-T_{z}\right\|<\varepsilon$ for all $z \in \mathbb{D}$ and $T_{z} \rightarrow T_{x}$ strongly when $z \rightarrow x$. 
Corollary 4.6 If $S \in \mathfrak{L}\left(L_{a}^{2}\right)$ and $n \geq 0$ is an integer then $B_{n} S \in \mathcal{A}$. Besides, $B_{n} S_{x}=\left(B_{n} S\right) \circ \varphi_{x}$ for every $x \in M(\mathcal{A})$.

Proof. By (2.1) and Lemma 2.2

$$
\left(B_{n} S\right)(z)=\left(\left(B_{n} S\right) \circ \varphi_{z}\right)(0)=\left(B_{n} S_{z}\right)(0)=(n+1) \sum_{j=0}^{n}\left(\begin{array}{l}
n \\
j
\end{array}\right)(-1)^{j}\left\langle S_{z} \omega^{j}, \omega^{j}\right\rangle .
$$

Since by Proposition 4.4 the map $z \mapsto\left\langle S_{z} \omega^{j}, \omega^{j}\right\rangle$ extends continuously to $M(\mathcal{A})$, it belongs to $\mathcal{A}$ for every $0 \leq j \leq n$. For the second assertion take a net $\left(z_{\alpha}\right)$ in $\mathbb{D}$ that tends to $x$ and then take limits in the equality $\left(B_{n} S_{z_{\alpha}}\right)(\xi)=\left(B_{n} S\right)\left(\varphi_{z_{\alpha}}(\xi)\right)$ for each fixed $\xi \in \mathbb{D}$. The first term tends to $\left(B_{n} S_{x}\right)(\xi)$ because Proposition 4.4 says that

$$
z \mapsto\left\langle S_{z} \omega^{j} K_{\xi}^{(n)}, \omega^{j} K_{\xi}^{(n)}\right\rangle
$$

extends continuously to $M(\mathcal{A})$, and the second term tends to $\left(B_{n} S\right)\left(\varphi_{x}(\xi)\right)$ because $B_{n} S \in \mathcal{A}$.

Corollary 4.7 If $S \in \mathfrak{L}\left(L_{a}^{2}\right)$ and $x \in M(\mathcal{A})$ the following conditions are equivalent

(i) $S_{u}=\lambda I$ for every $u \in H(x)$

(ii) $S_{u}=\lambda I$ for some $u \in H(x)$

(iii) $B_{0} S \equiv \lambda$ on $H(x)$.

Proof. Since $H(u)=H(x)$ when $u \in H(x)$ then every $v \in H(x)$ has the form $v=\varphi_{u}(\omega)$ for some $\omega \in \mathbb{D}$. By the previous corollary

$$
\left(B_{0} S\right)(v)=\left(B_{0} S\right)\left(\varphi_{u}(\omega)\right)=\left(B_{0} S_{u}\right)(\omega) .
$$

This identity and the fact that $B_{0}$ acts in a one-to-one fashion on $\mathfrak{L}\left(L_{a}^{2}\right)$ give all the equivalences.

Since for $a \in \mathcal{A}$ we have

$$
\left(T_{a}\right)_{z}^{*}=T_{\bar{a} \circ \varphi_{z}} \rightarrow T_{\bar{a} \circ \varphi_{x}}=\left(T_{a}\right)_{x}^{*}
$$

in the SOT-topology when $z \rightarrow x$, then also $\left(T_{z}\right)^{*} \rightarrow\left(T_{x}\right)^{*}$ in the SOT-topology for all $T \in \mathfrak{T}(\mathcal{A})$. Also, since the product of a WOT-convergent and a SOT-convergent net in $\mathfrak{L}\left(L_{a}^{2}\right)$ tends weakly to the product of the limits, Proposition 4.4 and Theorems 4.5 imply that

$$
S_{x} T_{x}=(S T)_{x} \text { and } T_{x} S_{x}=(T S)_{x}
$$

for every $S \in \mathfrak{L}\left(L_{a}^{2}\right), T \in \mathfrak{T}(\mathcal{A})$ and $x \in M(\mathcal{A})$. This fails if we only assume $S, T \in \mathfrak{L}\left(L_{a}^{2}\right)$. 
Indeed, consider the operator defined by $S f(\omega)=f(-\omega)$. Since $S^{2}=I$ then $\left(S^{2}\right)_{x}=I$ for every $x \in M(\mathcal{A})$. On the other hand, since $S K_{z}^{(0)}=K_{-z}^{(0)}$ then

$$
\left(B_{0} S\right)(z)=\left(1-|z|^{2}\right)^{2}\left\langle K_{-z}^{(0)}, K_{z}^{(0)}\right\rangle=\frac{\left(1-|z|^{2}\right)^{2}}{\left(1+|z|^{2}\right)^{2}} .
$$

So $\left(B_{0} S\right)(z) \rightarrow 0$ when $|z| \rightarrow 1$, and then $\left(B_{0} S\right)(x)=0$ for all $x \in M(\mathcal{A}) \backslash \mathbb{D}$. Corollary 4.7 then tells us that $S_{x}=0$ for $x \in M(\mathcal{A}) \backslash \mathbb{D}$, making (4.2) impossible for this choice of $S$ and $T=S$.

Lemma 4.8 Let $S \in \mathfrak{L}\left(L_{a}^{2}\right)$ and $x \in M(\mathcal{A})$. Suppose that there is some $n_{0} \geq 0$ such that $\left(B_{n_{0}} S\right) \circ \varphi_{x}=g$ harmonic. Then $\left(B_{n} S\right) \circ \varphi_{x}=g$ for every $n \geq 0$.

Proof. By Corollary 4.6, $\tilde{\Delta}\left(B_{n_{0}} S_{x}\right)=\tilde{\Delta} g=0$, which together with (2.7) yields $B_{n_{0}+1} S_{x}=B_{n_{0}} S_{x}=g$. Then $B_{n} S_{x}=g$ for every $n \geq n_{0}$. Thus $B_{0}\left(B_{n} S_{x}\right)=B_{0} g=g$ for $n \geq n_{0}$, implying that

$$
g=\lim _{n \rightarrow \infty} B_{0} B_{n} S_{x}=\lim _{n \rightarrow \infty} B_{n} B_{0} S_{x}=B_{0} S_{x},
$$

where the second equality follows from Corollary 2.7 and the last one because since $B_{0} S_{x} \in \mathcal{A}$ by Corollary 4.6, then $B_{n}\left(B_{0} S_{x}\right) \rightarrow B_{0} S_{x}$ uniformly. Taking $n_{0}=0$, we have proved above that $B_{n} S_{x}=g$ for every $n \geq 0$.

By the lemma we can add two more equivalences to Corollary 4.7, saying that $B_{n} S \equiv \lambda$ on $H(x)$ for every (or for some) $n \geq 0$.

Theorem 4.9 Let $S \in \mathfrak{T}(\mathcal{A})$ and $\mathcal{B}$ be a hyperbolic algebra. Then the following conditions are equivalent,

(1) $S_{x}=\lambda I$ when $x \in \pi^{-1}(y)$ for every $y \in \Gamma_{\mathcal{B}}$, where $\lambda \in \mathbb{C}$ depends only on $y$,

(2) there is a continuous map $\Psi_{S}^{\mathcal{B}}: M(\mathcal{B}) \rightarrow(\mathfrak{T}(\mathcal{A})$, SOT $)$ such that $\Psi_{S}^{\mathcal{B}} \circ \pi=\Psi_{S}$

(3) $B_{n} S \in \mathcal{B}$ for some $n \geq 0$,

(4) $B_{n} S \in \mathcal{B}$ for all $n \geq 0$.

If $S \in \mathfrak{L}\left(L_{a}^{2}\right)$ the theorem holds replacing $(\mathfrak{T}(\mathcal{A}), S O T)$ by $\left(\mathfrak{L}\left(L_{a}^{2}\right)\right.$,WOT) in $(2)$.

Proof. If (1) holds then for every $y \in M(\mathcal{B})$ and $x \in \pi^{-1}(y), S_{x}$ is an operator that only depends on $y$. Hence $\Psi_{S}^{\mathcal{B}}(y)=S_{x}$ is well defined and satisfies the equality in (2). The continuity of $\Psi_{S}^{\mathcal{B}}$ from $M(\mathcal{B})$ into any of the metric spaces $(\mathfrak{T}(\mathcal{A}), \mathrm{SOT})$ or $\left(\mathfrak{L}\left(L_{a}^{2}\right), \mathrm{WOT}\right)$ (according to the hypothesis) follows from the respective continuity of $\Psi_{S}$, which is given by Theorem 4.5 and Proposition 4.4. 
Now suppose that (2) holds. This means that if $y \in M(\mathcal{B})$ then $S_{x}$ is the same operator $T$ for every $x \in \pi^{-1}(y)$. Since $\varphi_{x}(\mathbb{D}) \subset \pi^{-1}(y)$ for $y \in \Gamma_{\mathcal{B}}$, then $S_{\varphi_{x}(\omega)}=T$ for every $\omega \in \mathbb{D}$. Corollary 4.6 then says that

$$
\left(B_{0} S\right)\left(\varphi_{x}(\omega)\right)=\left(B_{0} S_{\varphi_{x}(\omega)}\right)(0)=\left(B_{0} T\right)(0)
$$

for every $x \in \pi^{-1}(y)$ and $\omega \in \mathbb{D}$. Writing $\lambda=\left(B_{0} T\right)(0)$, we obtain that $B_{0} S \equiv \lambda$ on $H(x)$ for every $x \in \pi^{-1}(y)$. Hence $B_{0} S$ is constant on $\pi^{-1}(y)$ for every $y \in \Gamma_{\mathcal{B}}$, meaning that $\left.\left(B_{0} S\right)\right|_{D}$ extends continuously to $M(\mathcal{B})$. Since the Gelfand-Naimark Theorem identifies $\mathcal{B}$ with $C(M(\mathcal{B}))$ then $B_{0} S \in \mathcal{B}$. This proves (3) for $n=0$. If (3) holds for some $n_{0} \geq 0$ then $B_{n_{0}} S=$ $\lambda_{y} \in \mathbb{C}$ on $\pi^{-1}(y)$ for every $y \in \Gamma_{\mathcal{B}}$. Lemma 4.8 then implies that the same happens with $B_{n} S$ for all $n \geq 0$. This proves (4). Finally, if (4) holds then $\left.\left(B_{0} S\right)\right|_{\pi^{-1}(y)}=\lambda_{y} \in \mathbb{C}$ for $y \in \Gamma_{\mathcal{B}}$. In particular, $B_{0} S \equiv \lambda_{y}$ on $H(x)$ for every $x \in \pi^{-1}(y)$. Then (1) follows from Corollary 4.7 .

If $S \in \mathfrak{L}\left(L_{a}^{2}\right)$ satisfies the conditions of the theorem then the map $z \mapsto S_{z}$ admits a continuous extension to $M(\mathcal{B})$ given by $\Psi_{S}^{\mathcal{B}}$. Write

$$
\Psi_{S}^{\mathcal{B}}(y)=\widehat{S}_{y}^{\mathcal{B}}
$$

when $y \in M(\mathcal{B})$. If $\mathcal{B}=\mathcal{A}$ we keep the previous notation $\Psi_{S}(y)=S_{y}$ for $y \in M(\mathcal{A})$. Also, since it is clear that we can identify $\widehat{S}_{z}^{\mathcal{B}}$ with $S_{z}$ when $z \in \mathbb{D}$, we do not make this notation distinction for $z \in \mathbb{D}$. Observe that if $y \in M(\mathcal{B})$ and $\left(z_{\alpha}\right)$ is a net in $\mathbb{D}$ that tends to $y$ in $M(\mathcal{B})$, then $\widehat{S}_{y}^{\mathcal{B}}$ admits the two alternative and equivalent expressions

$$
\widehat{S}_{y}^{\mathcal{B}}=\lim _{\alpha} S_{z_{\alpha}}
$$

a WOT-limit in general and a $\mathrm{SOT}$-limit if $S \in \mathfrak{T}(\mathcal{A})$, or

$$
\widehat{S}_{y}^{\mathcal{B}}=S_{x} \text { for some (or all) } x \in \pi^{-1}(y),
$$

where $\pi: M(\mathcal{A}) \rightarrow M(\mathcal{B})$ is the natural projection. Also, if $b \in \mathcal{B}$ we can look at $b$ as a continuous function on $M(\mathcal{B})$ or on $M(\mathcal{A})$. If $\mathcal{B} \neq \mathcal{A}$ we write $\widehat{b}^{\mathcal{B}}$ when we need to distinguish the domain of the function, otherwise $b$ will be looked as a function on $M(\mathcal{A})$. Of course, if $z \in \mathbb{D}$ then $b(z)$ has the same meaning either way.

If $\mathcal{B}$ is a hyperbolic algebra, $b \in \mathcal{B}$ and $y \in \Gamma_{\mathcal{B}}$, then for every $x \in \pi^{-1}(y)$ we have

$$
\left(T_{b}\right)_{x}=T_{b \circ \varphi_{x}}=\lambda I
$$

with $\lambda \in \mathbb{C}$ depending only on $y$ (actually $\lambda=\widehat{b}^{\mathcal{B}}(y)$ ). Since $\mathfrak{T}(\mathcal{B})$ is generated by these Toeplitz operators, the same holds for every $S \in \mathfrak{T}(\mathcal{B})$. Theorem 4.9 then says that $B_{n} S \in \mathcal{B}$ when $S \in \mathfrak{T}(\mathcal{B})$, for every nonnegative integer $n$. 


\section{Approximation and truncation by Toeplitz operators}

If $A \subset L^{\infty}(\mathbb{D})$ is a subalgebra, we write $\mathfrak{T}_{0}(A)$ for the algebra generated by the Toeplitz operators $T_{a}$, with $a \in A$, without taking closure. In [4] Axler and Zheng found simple but very ingenious estimates for the norm of operators in $\mathfrak{T}_{0}\left(L^{\infty}(\mathbb{D})\right.$ ). The present section (especially Lemmas 5.2 and 5.5) makes heavy use of their method.

\subsection{Norm estimates and truncation}

The following lemma is a particular case of Lemma 4.2.2 in [21].

Lemma 5.1 If $c<0$ and $t>-1$ then

$$
J_{c, t}(z)=\int_{D} \frac{\left(1-|\omega|^{2}\right)^{t}}{|1-z \bar{\omega}|^{2+t+c}} d A(\omega), \quad z \in \mathbb{D},
$$

is bounded.

The next result appeared in [4] for $p=6$. The proof sketched here is a standard modification of that proof involving Lemma 5.1.

Lemma 5.2 Let $p>4$. Then there is a constant $C_{p}<\infty$ such that if $S \in \mathfrak{L}\left(L_{a}^{2}\right)$, then

$$
\int_{D} \frac{\left|\left(S K_{z}^{(0)}\right)(w)\right|}{\sqrt{1-|w|^{2}}} d A(w) \leq \frac{C_{p}\left\|S_{z} 1\right\|_{p}}{\sqrt{1-|z|^{2}}}
$$

for all $z \in \mathbb{D}$ and

$$
\int_{D} \frac{\left|\left(S K_{z}^{(0)}\right)(w)\right|}{\sqrt{1-|z|^{2}}} d A(z) \leq \frac{C_{p}\left\|S_{w}^{*} 1\right\|_{p}}{\sqrt{1-|w|^{2}}}
$$

for all $w \in \mathbb{D}$.

Proof. To prove (5.1) let $S \in \mathfrak{L}\left(L_{a}^{2}\right)$ and fix $z \in \mathbb{D}$. Since

$$
U_{z} 1=\left(|z|^{2}-1\right) K_{z}^{(0)} \quad \text { and } \quad U_{z} U_{z}=I
$$

then

$$
U_{z} S_{z} 1=\left(|z|^{2}-1\right) S K_{z}^{(0)} .
$$

Thus

$$
\int_{D} \frac{\left|\left(S K_{z}^{(0)}\right)(w)\right|}{\sqrt{1-|w|^{2}}} d A(w)=\frac{1}{1-|z|^{2}} \int_{D} \frac{\left|\left(S_{z} 1\right)\left(\varphi_{z}(w)\right)\right|\left|\varphi_{z}^{\prime}(w)\right|}{\sqrt{1-|w|^{2}}} d A(w) .
$$


Making the substitution $w=\varphi_{z}(\lambda)$ in the last integral and using Holder's inequality with $1 / p+1 / q=1$, we obtain

$$
\begin{aligned}
\int_{D} \frac{\left|\left(S K_{z}^{(0)}\right)(w)\right|}{\sqrt{1-|w|^{2}}} d A(w) & =\frac{1}{\sqrt{1-|z|^{2}}} \int_{D} \frac{\left|\left(S_{z} 1\right)(\lambda)\right|}{|1-\bar{z} \lambda| \sqrt{1-|\lambda|^{2}}} d A(\lambda) \\
& \leq \frac{\left\|S_{z} 1\right\|_{p}}{\sqrt{1-|z|^{2}}}\left(\int_{D} \frac{d A(\lambda)}{|1-\bar{z} \lambda|^{q}\left(1-|\lambda|^{2}\right)^{q / 2}}\right)^{1 / q} \\
& =\frac{\left\|S_{z} 1\right\|_{p}}{\sqrt{1-|z|^{2}}} J(z)^{1 / q}
\end{aligned}
$$

where

$$
J(z)=\int_{D} \frac{\left(1-|\lambda|^{2}\right)^{-q / 2}}{|1-\bar{z} \lambda|^{2-(q / 2)+(3 / 2) q-2}} d A(\lambda) .
$$

Since $p>4$ then $q<4 / 3$, which yields $q / 2<2 / 3<1$ and $(3 / 2) q-2<0$. By Lemma 5.1 there is $J_{q}>0$ such that $J(z) \leq J_{q}$ for every $z \in \mathbb{D}$. This proves (5.1) with $C_{p}=J_{q}^{1 / q}$. Replace $S$ with $S^{*}$ and interchange the roles of $w$ and $z$ in (5.1) to obtain

$$
\int_{D} \frac{\left|\left(S^{*} K_{w}^{(0)}\right)(z)\right|}{\sqrt{1-|z|^{2}}} d A(z) \leq \frac{C_{p}\left\|S_{w}^{*} 1\right\|_{p}}{\sqrt{1-|w|^{2}}} .
$$

Then use the equality $\left(S^{*} K_{w}^{(0)}\right)(z)=\overline{\left(S K_{z}^{(0)}\right)(w)}$ to obtain $(5.2)$.

Lemma 5.3 Let $S \in \mathfrak{L}\left(L_{a}^{2}\right), a, b \in L^{\infty}(\mathbb{D})$ and $p>4$. Then

$$
\left\|T_{b} S T_{a}\right\|_{\mathcal{L}\left(L_{a}^{2}\right)} \leq C_{p}\left(\|a\|_{\infty}\|b\|_{\infty}\right)^{\frac{1}{2}} \sup _{z \in D}\left\{\left\|S_{z} 1\right\|_{p}|a(z)|\right\}^{\frac{1}{2}} \sup _{\omega \in D}\left\{\left\|S_{w}^{*} 1\right\|_{p}|b(w)|\right\}^{\frac{1}{2}},
$$

where $C_{p}$ is the constant of Lemma 5.2.

Proof. For $f \in L_{a}^{2}$ and $w \in D$, we have

$$
\begin{aligned}
\left(S T_{a} f\right)(w) & =\left\langle S T_{a} f, K_{w}^{(0)}\right\rangle=\left\langle a f, S^{*} K_{w}^{(0)}\right\rangle \\
& =\int_{D} f(z) a(z) \overline{\left(S^{*} K_{w}^{(0)}\right)(z)} d A(z) \\
& =\int_{D} f(z) a(z)\left(S K_{z}^{(0)}\right)(w) d A(z) .
\end{aligned}
$$

Thus, if $M_{b}$ denotes the multiplication operator,

$$
\left(M_{b} S T_{a}\right) f(w)=\int_{D} f(z) a(z) b(w)\left(S K_{z}^{(0)}\right)(w) d A(z) .
$$


If $\Phi(z, w)=\left|a(z) b(w)\left(S K_{z}^{(0)}\right)(w)\right|$ and $h(z)=\left(1-|z|^{2}\right)^{-1 / 2}$ then (5.1) yields

$$
\begin{aligned}
\int_{D} \Phi(z, w) h(w) d A(w) & \leq C_{p}\|b\|_{\infty}\left\|S_{z} 1\right\|_{p}|a(z)| h(z) \\
& \leq C_{p}\|b\|_{\infty} \sup _{z \in D}\left\{\left\|S_{z} 1\right\|_{p}|a(z)|\right\} h(z)
\end{aligned}
$$

and by (5.2)

$$
\begin{aligned}
\int_{D} \Phi(z, w) h(z) d A(w) & \leq C_{p}\|a\|_{\infty}\left\|S_{w}^{*} 1\right\|_{p}|b(w)| h(w) \\
& \leq C_{p}\|a\|_{\infty} \sup _{\omega \in D}\left\{\left\|S_{w}^{*} 1\right\|_{p}|b(w)|\right\} h(w) .
\end{aligned}
$$

By Schur's theorem (see the proof in [21, p. 42]) the operator $M_{b} S T_{a}$ satisfies an inequality as in the lemma. The result follows because

$$
\left\|\left(T_{b} S T_{a}\right) f\right\|_{L^{2}} \leq\left\|\left(M_{b} S T_{a}\right) f\right\|_{L^{2}}
$$

for every $f \in L_{a}^{2}$.

Suppose that $1<p<p^{\prime}<\infty, f \in L^{p}(\mathbb{D})$ and $0<r<1$. Split the integral

$$
\|f\|_{p}^{p}=\left\|f \chi_{D \backslash r D}\right\|_{p}^{p}+\left\|f \chi_{r D}\right\|_{p}^{p}
$$

where $\chi_{E}$ denotes the characteristic function of the set $E$. Taking $\alpha=p^{\prime} / p$ and $\beta=p^{\prime} /\left(p^{\prime}-p\right)$ we have $\alpha^{-1}+\beta^{-1}=1$. By Holder's inequality

$$
\left\|f \chi_{D \backslash r D}\right\|_{p}^{p} \leq\|f\|_{\alpha p}^{p}\left\|\chi_{D \backslash r D}\right\|_{\beta}=\|f\|_{p^{\prime}}^{p}\left(1-r^{2}\right)^{1-\frac{p}{p^{\prime}}},
$$

and consequently

$$
\|f\|_{p}^{p} \leq\|f\|_{p^{\prime}}^{p}\left(1-r^{2}\right)^{1-\frac{p}{p^{\prime}}}+\left\|f \chi_{r D}\right\|_{p}^{p}
$$

Proposition 5.4 Suppose that $S \in \mathfrak{T}_{0}\left(L^{\infty}(\mathbb{D})\right)$ and $F \subset M(\mathcal{A})$ is a closed saturated set such that $B_{0} S \equiv 0$ on $F$. Given $\varepsilon>0$ there is an open neighborhood $\Omega$ of $F$ in $M(\mathcal{A})$ such that if $U \subset \Omega \cap \mathbb{D}$ is measurable, then

$$
\left\|T_{a \chi_{U}} S\right\|_{\mathfrak{L}\left(L_{a}^{2}\right)}<\varepsilon \text { and }\left\|S T_{a \chi_{U}}\right\|_{\mathfrak{L}\left(L_{a}^{2}\right)}<\varepsilon
$$

for every $a \in L^{\infty}(\mathbb{D})$ with $\|a\|_{\infty} \leq 1$.

Proof. Since $F$ is saturated and $B_{0} S \equiv 0$ on $F$, Proposition 4.4 and Corollary 4.7 say that $S_{z} \stackrel{\text { woT }}{\rightarrow} S_{x}=0$ when $z \rightarrow x \in F$, with $z \in \mathbb{D}$. Thus $S_{z} 1 \rightarrow 0$ weakly in $L_{a}^{2}$ and consequently

$$
S_{z} 1 \rightarrow 0 \text { uniformly on compact sets as } z \rightarrow x \quad(z \in \mathbb{D})
$$

for every $x \in F$. 
Write

$$
S=\sum_{i=1}^{m} \prod_{j=1}^{n_{i}} T_{a_{j}^{i}}
$$

with $a_{j}^{i} \in L^{\infty}(\mathbb{D})$, and fix $p, p^{\prime}$ with $4<p<p^{\prime}$. Then

$$
\left\|S_{z} 1\right\|_{p^{\prime}}=\left\|\sum_{i=1}^{m} \prod_{j=1}^{n_{i}} T_{a_{j}^{i} \circ \varphi_{z}} 1\right\|_{p^{\prime}} \leq \sum_{i=1}^{m} \prod_{j=1}^{n_{i}} c_{p^{\prime}}\left\|a_{j}^{i}\right\|_{\infty}=c
$$

where $c_{p^{\prime}}$ is the norm of the analytic projection $P_{+}$acting on $L^{p^{\prime}}(\mathbb{D})$. For $0<r<1$, (5.3) yields

$$
\left\|S_{z} 1\right\|_{p}^{p} \leq\left\|S_{z} 1\right\|_{p^{\prime}}^{p}(1-r)^{1-\frac{p}{p^{\prime}}}+\left\|\left(S_{z} 1\right) \chi_{r D}\right\|_{p}^{p} .
$$

By (5.6) there is $r$ close enough to 1 so that the first member of the sum is smaller than $\varepsilon / 2$, while (5.5) and the compactness of $F$ imply that there is a neighborhood $\Omega$ of $F$ so that the second member is smaller than $\varepsilon / 2$ for every $z \in \Omega \cap \mathbb{D}$. In particular, if $U \subset \Omega \cap \mathbb{D}$ this holds for every $z \in U$. Since $\|a\|_{\infty} \leq 1$, Lemma 5.3 gives

$$
\left\|S T_{a \chi_{U}}\right\|^{2} \leq C_{p}^{2} \sup \left\{\left\|S_{z} 1\right\|_{p}: z \in U\right\} \sup _{D}\left\|S_{\omega}^{*} 1\right\|_{p} \leq C_{p}^{2} c \varepsilon^{1 / p},
$$

where $c$ comes from (5.6) with $S^{*}$ instead of $S$, and $C_{p}$ is the constant of Lemma 5.3. To prove the first inequality of (5.4) observe that $B_{0} S^{*}=\overline{B_{0} S}$ also satisfies the hypothesis of the proposition and $\left\|T_{a \chi_{U}} S\right\|=\left\|S^{*} T_{\bar{a} \chi_{U}}\right\|$.

\subsection{Approximation properties of the $k$-Berezin transforms}

Lemma 5.5 Suppose that $\left\{S_{k}\right\}$ is a bounded sequence in $\mathfrak{L}\left(L_{a}^{2}\right)$ such that $\left\|B_{0} S_{k}\right\|_{\infty} \rightarrow 0$ when $k \rightarrow \infty$. Then

$$
\sup _{z \in D}\left|\left(S_{k}\right)_{z} 1\right| \rightarrow 0
$$

uniformly on compact subsets of $\mathbb{D}$ when $k \rightarrow \infty$.

Proof. Since there is a constant $C$ such that $\left\|S_{k}\right\| \leq C$ for every $k$, then it is enough to prove that for every $S \in \mathfrak{L}\left(L_{a}^{2}\right), \eta>0$ and $r \in(0,1)$, there is a function $c(r, \eta)>0$, independent of $S$, such that

$$
\sup _{z \in D}\left|\left(S_{z} 1\right)(u)\right| \leq c(r, \eta)\left\|B_{0} S\right\|_{\infty}+\eta\|S\|
$$

when $u \in r \mathbb{D}$. 
Since

$$
K_{z}^{(0)}(w)=\sum_{m=0}^{\infty}(m+1) \bar{z}^{m} \omega^{m},
$$

then for $z, \lambda \in \mathbb{D}$ we have

$$
\left(B_{0} S\right)\left(\varphi_{z}(\lambda)\right)=\left(B_{0} S_{z}\right)(\lambda)=\left(1-|\lambda|^{2}\right)^{2} \sum_{j, m=0}^{\infty}(j+1)(m+1)\left\langle S_{z} \omega^{j}, \omega^{m}\right\rangle \bar{\lambda}^{j} \lambda^{m},
$$

where the first equality comes from Lemma 2.2. Then, for $0<\delta<1 / 2$ (to be chosen later) we obtain

$$
\begin{aligned}
\int_{\delta D} \frac{\left(B_{0} S\right)\left(\varphi_{z}(\lambda)\right) \bar{\lambda}^{n}}{\left(1-|\lambda|^{2}\right)^{2}} d A(\lambda) & =\sum_{j, m=0}^{\infty}(j+1)(m+1)\left\langle S_{z} \omega^{j}, \omega^{m}\right\rangle \int_{\delta D} \bar{\lambda}^{j+n} \lambda^{m} d A(\lambda) \\
& =\sum_{j=0}^{\infty}(j+1)\left\langle S_{z} \omega^{j}, \omega^{j+n}\right\rangle \delta^{2 j+2 n+2} \\
& =\delta^{2 n+2}\left(\left\langle S_{z} 1, \omega^{n}\right\rangle+\sum_{j=1}^{\infty}(j+1)\left\langle S_{z} \omega^{j}, \omega^{j+n}\right\rangle \delta^{2 j}\right) .
\end{aligned}
$$

Since $0<\delta<1 / 2$ and $\left\|\omega^{j}\right\|=(j+1)^{-1 / 2}$ then

$$
\begin{aligned}
\left|\left\langle S_{z} 1, \omega^{n}\right\rangle\right| & \leq \frac{1}{\delta^{2 n+2}}\left\|B_{0} S\right\|_{\infty} \int_{\delta D} \frac{\delta^{n} d A(\lambda)}{\left(1-|\lambda|^{2}\right)^{2}}+\|S\| \sum_{j=1}^{\infty}(j+1)\left\|\omega^{j}\right\|\left\|\omega^{j+n}\right\| \delta^{2 j} \\
(5.9) & \leq 2 \delta^{-n}\left\|B_{0} S\right\|_{\infty}+\delta\|S\|,
\end{aligned}
$$

where the last inequality holds because $\sum_{j=1}^{\infty} \delta^{2 j} \leq \delta$ when $0<\delta<1 / 2$. By (5.8)

$$
\left(S_{z} 1\right)(u)=\left\langle S_{z} 1, K_{u}^{(0)}\right\rangle=\sum_{n \geq 0}(n+1)\left\langle S_{z} 1, \omega^{n}\right\rangle u^{n},
$$

implying that

$$
\left|\left(S_{z} 1\right)(u)\right| \leq \sum_{0 \leq n \leq N-1}(n+1)\left|\left\langle S_{z} 1, \omega^{n}\right\rangle\right|+\sum_{n \geq N}(n+1)^{1 / 2}\left\|S_{z}\right\| r^{n}
$$

for $z \in \mathbb{D}, u \in r \mathbb{D}$ and $N \geq 1$. Since $r \in(0,1)$ we can fix some integer $N=N(r, \eta)$ big enough so that the second sum is bounded by $(\eta / 2)\|S\|$. Using (5.9) in (5.10) we get

$$
\begin{aligned}
\left|\left(S_{z} 1\right)(u)\right| & \leq N \sum_{0 \leq n \leq N-1}\left|\left\langle S_{z} 1, \omega^{n}\right\rangle\right|+(\eta / 2)\|S\| \\
& \leq 2 N^{2} \delta^{-N}\left\|B_{0} S\right\|_{\infty}+N^{2} \delta\|S\|+(\eta / 2)\|S\|
\end{aligned}
$$

for $z \in \mathbb{D}$ and $u \in r \mathbb{D}$. Choosing $\delta=\delta(r, \eta)<\min \left\{\eta / 2 N^{2}, 1 / 2\right\}$ we obtain $(5.7)$ with $c(r, \eta)=2 N^{2} \delta^{-N}$. 
Lemma 5.6 Let $\left\{S_{k}\right\}$ be a sequence in $\mathfrak{L}\left(L_{a}^{2}\right)$ such that for some $p^{\prime}>4$,

$$
\begin{gathered}
\left\|B_{0} S_{k}\right\|_{\infty} \rightarrow 0, \text { when } k \rightarrow \infty, \\
\sup _{z \in D}\left\|\left(S_{k}\right)_{z} 1\right\|_{p^{\prime}} \leq C \text { and } \sup _{\omega \in D}\left\|\left(S_{k}^{*}\right)_{\omega} 1\right\|_{p^{\prime}} \leq C,
\end{gathered}
$$

where $C>0$ does not depend on $k$. Then

$$
\left\|S_{k}\right\|_{\mathfrak{L}\left(L_{a}^{2}\right)} \rightarrow 0 \quad \text { when } k \rightarrow \infty
$$

Proof. By (5.12) and Lemma 5.3 with $a=b=1$,

$$
\left\|S_{k}\right\|_{\mathfrak{L}\left(L_{a}^{2}\right)} \leq C_{p^{\prime}} \sup _{z \in D}\left\{\left\|\left(S_{k}\right)_{z} 1\right\|_{p^{\prime}}\right\}^{1 / 2} \sup _{\omega \in D}\left\{\left\|\left(S_{k}^{*}\right)_{w} 1\right\|_{p^{\prime}}\right\}^{1 / 2} \leq C_{p^{\prime}} C .
$$

Hence, $\left\{S_{k}\right\}$ is a bounded sequence in $\mathfrak{L}\left(L_{a}^{2}\right)$ that satisfies (5.11). Under these conditions Lemma 5.5 says that

$$
\sup _{z \in D}\left|\left(S_{k}\right)_{z} 1\right| \rightarrow 0 \quad \text { uniformly on compact sets of } \mathbb{D} .
$$

Let $p$ with $4<p<p^{\prime}$. By (5.3)

$$
\sup _{z \in D}\left\|\left(S_{k}\right)_{z} 1\right\|_{p}^{p} \leq \sup _{z \in D}\left\|\left(S_{k}\right)_{z} 1\right\|_{p^{\prime}}^{p}(1-r)^{1-\frac{p}{p^{\prime}}}+\sup _{z \in D}\left\|\left[\left(S_{k}\right)_{z} 1\right] \chi_{r D}\right\|_{p}^{p}
$$

for every $0<r<1$. By (5.12) the first member of the sum is bounded by

$$
C^{p}(1-r)^{1-\frac{p}{p^{\prime}}}
$$

which can be made small by taking $r$ close to 1 , and by (5.13) the second member of the sum tends to 0 as $k \rightarrow \infty$. Therefore,

$$
\sup _{z \in D}\left\|\left(S_{k}\right)_{z} 1\right\|_{p} \rightarrow 0 \quad \text { when } k \rightarrow \infty
$$

for every $p \in\left(4, p^{\prime}\right)$. Using again Lemma 5.3 , this time with $p$ instead of $p^{\prime}$, we obtain

$$
\begin{aligned}
\left\|S_{k}\right\|_{\mathcal{L}\left(L_{a}^{2}\right)} & \leq C_{p} \sup _{z \in D}\left\{\left\|\left(S_{k}\right)_{z} 1\right\|_{p}\right\}^{1 / 2} \sup _{\omega \in D}\left\{\left\|\left(S_{k}^{*}\right)_{w} 1\right\|_{p}\right\}^{1 / 2} \\
& \leq C_{p} \sup _{z \in D}\left\{\left\|\left(S_{k}\right)_{z} 1\right\|_{p}\right\}^{1 / 2} C^{1 / 2} \rightarrow 0
\end{aligned}
$$

when $k \rightarrow \infty$, where the last inequality holds by (5.12), since \|\|$_{p} \leq\|\|_{p^{\prime}}$. 
Theorem 5.7 If $a \in L^{\infty}(\mathbb{D})$ then $T_{B_{k}(a)} \rightarrow T_{a}$ in operator norm when $k \rightarrow \infty$. In particular, $\mathfrak{T}(\mathcal{A})=\mathfrak{T}\left(L^{\infty}(\mathbb{D})\right)$.

Proof. Write $S_{k}=T_{B_{k}(a)}-T_{a}$. Since Corollary 2.7 says that $B_{0} B_{k}=B_{k} B_{0}$ on $\mathfrak{L}\left(L_{a}^{2}\right)$ then

$$
B_{0} S_{k}=B_{0} T_{B_{k}(a)}-B_{0} T_{a}=B_{0} B_{k}(a)-B_{0}(a)=B_{k} B_{0}(a)-B_{0}(a),
$$

which tends uniformly to 0 when $k \rightarrow \infty$ because $B_{0}(a) \in \mathcal{A}$. That is, $\left\{S_{k}\right\}$ satisfies (5.11). On the other hand, if $p^{\prime}>4$ then

$$
\left\|\left(S_{k}\right)_{z} 1\right\|_{p^{\prime}}=\left\|P_{+} M_{\left(B_{k}(a)-a\right) \circ \varphi_{z}} 1\right\|_{p^{\prime}} \leq c_{p^{\prime}}\left(\left\|B_{k}(a)\right\|_{\infty}+\|a\|_{\infty}\right) \leq 2 c_{p^{\prime}}\|a\|_{\infty},
$$

where $c_{p^{\prime}}$ is the norm of the analytic projection $P_{+}$acting on $L^{p^{\prime}}(\mathbb{D})$ (see $[21$, p. 54]). Since

then also

$$
\left(S_{k}^{*}\right)_{z}=P_{+} M_{\left(\overline{B_{k}(a)}-\bar{a}\right) \circ \varphi_{z}}
$$

$$
\left\|\left(S_{k}^{*}\right)_{z} 1\right\|_{p^{\prime}} \leq 2 c_{p^{\prime}}\|a\|_{\infty}
$$

So, $\left\{S_{k}\right\}$ satisfies (5.12) and Lemma 5.6 then says that $\left\|S_{k}\right\|_{\mathfrak{L}\left(L_{a}^{2}\right)} \rightarrow 0$ as $k \rightarrow \infty$.

Remark 5.8 An obvious consequence of the theorem is that Theorems 4.5 and 4.9 hold for $S \in \mathfrak{T}\left(L^{\infty}(\mathbb{D})\right)$. The argument of Theorem 5.7 works word by word for any $S \in \mathfrak{L}\left(L_{a}^{2}\right)$ such that $T_{B_{k} S}-S$ satisfies (5.12) for some $p^{\prime}>4$. So, $T_{B_{k} S} \rightarrow S$ for such $S$. Maybe this holds for every $S \in \mathfrak{T}_{0}\left(L^{\infty}(\mathbb{D})\right)$, which would imply that $\mathfrak{T}\left(L^{\infty}(\mathbb{D})\right)$ is the closure of $\left\{T_{a}: a \in \mathcal{A}\right\}$.

\section{Abelianization}

Lemma 6.1 Let $F \subset M(\mathcal{A}) \backslash \mathbb{D}$ be a closed saturated set, $\Omega \subset M(\mathcal{A})$ an open neighborhood of $F$ and $k \geq 0$ an integer. Write $U=\Omega \cap \mathbb{D}$ and $\mathfrak{F}=\left\{a \in L^{\infty}(\mathbb{D}): a \equiv 0\right.$ on $\left.U\right\}$. Then

$$
B_{k} a \equiv 0 \text { on } F \text { for every } a \in \mathfrak{F} \text {. }
$$

In particular, if $\mathcal{B}$ is a hyperbolic algebra and $F=\pi^{-1}\left(\Gamma_{\mathcal{B}}\right)$ then $B_{k} a \in \mathcal{B}$ and $T_{a} \in \mathfrak{T}(\mathcal{B})$.

Proof. By Lemma 4.8 it is enough to prove the lemma for $k=0$. Let $x \in F$ and take a net $\left(z_{\alpha}\right)$ in $\mathbb{D}$ such that $z_{\alpha} \rightarrow x$. We claim that for every $r \in(0,1)$ there is $\alpha_{0}=\alpha_{0}(r)$ such that $\varphi_{z_{\alpha}}(r \mathbb{D}) \subset \Omega$ for $\alpha \geq \alpha_{0}$. Otherwise there is a subnet $\left(z_{\alpha_{\beta}}\right)$ and points $\xi_{\beta} \in r \mathbb{D}$ such that $\varphi_{z_{\alpha_{\beta}}}\left(\xi_{\beta}\right) \notin \Omega$ for all $\beta$. We can assume that $\xi_{\beta} \rightarrow \xi_{0}$, with $\left|\xi_{0}\right| \leq r$. If $f \in \mathcal{A}$, the inequality $\left|f\left(\varphi_{z_{\alpha_{\beta}}}\left(\xi_{\beta}\right)\right)-f\left(\varphi_{x}\left(\xi_{\beta}\right)\right)\right| \leq\left|f\left(\varphi_{z_{\alpha_{\beta}}}\left(\xi_{\beta}\right)\right)-f\left(\varphi_{z_{\alpha_{\beta}}}\left(\xi_{0}\right)\right)\right|+\left|f\left(\varphi_{z_{\alpha_{\beta}}}\left(\xi_{0}\right)\right)-f\left(\varphi_{x}\left(\xi_{0}\right)\right)\right|$ and the uniform $\rho$-continuity of $f$ imply that $f\left(\varphi_{z_{\alpha_{\beta}}}\left(\xi_{\beta}\right)\right) \rightarrow f\left(\varphi_{x}\left(\xi_{0}\right)\right)$. 
Therefore

$$
\varphi_{z_{\alpha_{\beta}}}\left(\xi_{\beta}\right) \rightarrow \varphi_{x}\left(\xi_{0}\right) \in H(x) \subset F,
$$

and since $\Omega$ is a neighborhood of $F$ then $\varphi_{z_{\alpha_{\beta}}}\left(\xi_{\beta}\right) \in \Omega$ for $\beta \geq \beta_{0}$, a contradiction. So, if $a \in \mathfrak{F}$ and $0<r<1$, there is $\alpha_{0}$ such that $\left(a \circ \varphi_{z_{\alpha}}\right)(\omega)=0$ for $|\omega|<r$ and $\alpha \geq \alpha_{0}$. Hence for $\alpha \geq \alpha_{0}$,

$$
\left|\left(B_{0} a\right)\left(z_{\alpha}\right)\right| \leq \int_{D}\left|\left(a \circ \varphi_{z_{\alpha}}\right)(\omega)\right| d A(\omega)=\int_{D \backslash r D}\left|\left(a \circ \varphi_{z_{\alpha}}\right)(\omega)\right| d A(\omega) \leq\|a\|_{\infty}\left(1-r^{2}\right),
$$

which can be made arbitrarily small by taking $r$ close enough to 1 . Therefore $\left(B_{0} a\right)\left(z_{\alpha}\right) \rightarrow 0$, but since also $\left(B_{0} a\right)\left(z_{\alpha}\right) \rightarrow\left(B_{0} a\right)(x)$ then $\left(B_{0} a\right)(x)=0$, and this happens for all $x \in F$.

Now suppose that $F=\pi^{-1}\left(\Gamma_{\mathcal{B}}\right)$, with $\mathcal{B}$ a hyperbolic algebra. Since $B_{k} a \in \mathcal{A}$ identically vanishes on $\pi^{-1}\left(\Gamma_{\mathcal{B}}\right)$ then $B_{k} a \in \mathcal{B}$. Consequently $T_{B_{k} a} \in \mathfrak{T}(\mathcal{B})$, and since by Theorem $5.7, T_{B_{k} a} \rightarrow T_{a}$ as $k \rightarrow \infty$, then so is $T_{a}$.

Let $F \subset M(\mathcal{A})$ be a closed set. A set $U \subset \mathbb{D}$ will be called a relative neighborhood of $F$ if there is some open neighborhood $\Omega \subset M(\mathcal{A})$ of $F$ such that $U=\Omega \cap \mathbb{D}$. Since the disk is dense in $M(\mathcal{A})$ and $\Omega$ is open, it is clear that $\bar{U}^{M(\mathcal{A})}$ contains $\Omega$, and consequently it is a neighborhood of $F$. Also, for $V \subset \mathbb{D}$ we will denote $V^{c}=\mathbb{D} \backslash V$.

Lemma 6.2 Let $S=\sum_{i=1}^{m} \prod_{j=1}^{n_{i}} T_{a_{j}^{i}}$, with $a_{j}^{i} \in L^{\infty}(\mathbb{D})$ for $1 \leq i \leq m$ and $1 \leq j \leq n_{i}$, and $F \subset M(\mathcal{A})$ be a closed saturated set such that $B_{0} S \equiv 0$ on $F$. Then given $\varepsilon>0$ there exist relative neighborhoods $U, V$ of $F$ such that

$$
\left\|S-\left(\sum_{i=1}^{m} \prod_{j=1}^{n_{i}} T_{a_{j}^{i} \chi_{V^{c}}}\right) T_{\chi_{U^{c}}}\right\|<\varepsilon .
$$

Proof. Without loss of generality we can assume that $\left\|a_{j}^{i}\right\|_{\infty} \leq 1$ for every $i, j$. By Proposition 5.4 there is a relative neighborhood $U$ of $F$ such that

$$
\left\|S-S T_{\chi_{U^{c}}}\right\|=\left\|S T_{\chi_{U}}\right\|<\varepsilon .
$$

By Lemma 6.1 and (4.2), for $1 \leq i \leq m$ each of the operators

$$
S_{k}^{i} \stackrel{\text { def }}{=}\left(\prod_{j=k}^{n_{i}} T_{a_{j}^{i}}\right) T_{\chi_{U^{c}}}, 1 \leq k \leq n_{i}, \quad S_{n_{i}+1}^{i}=T_{\chi_{U^{c}}}
$$

satisfies $B_{0} S_{k}^{i}=0$ on $F$. Hence, a new use of Proposition 5.4 provides a relative neighborhood $V$ of $F$ such that

$$
\left\|T_{a_{k}^{i} \chi_{V}} S_{k+1}^{i}\right\| \leq \varepsilon
$$

for every $1 \leq i \leq m$ and $1 \leq k \leq n_{i}$. 
Indeed, the proposition says that there are relative neighborhoods $V_{k}^{i}$ of $F$ that satisfy the inequality for each $i$ and $k$, but it also says that their intersection satisfies the inequality. Therefore

$$
\begin{aligned}
\| T_{a_{1}^{i} \chi_{V^{c}}} \ldots & T_{a_{k-1}^{i} \chi_{V^{c}}} S_{k}^{i}-T_{a_{1}^{i} \chi_{V^{c}}} \ldots T_{a_{k}^{i} \chi_{V^{c}}} S_{k+1}^{i} \| \\
& =\left\|T_{a_{1}^{i} \chi_{V^{c}}} \ldots T_{a_{k-1}^{i} \chi_{V^{c}}} T_{a_{k}^{i}} S_{k+1}^{i}-T_{a_{1}^{i} \chi_{V^{c}}} \ldots T_{a_{k}^{i} \chi_{V^{c}}} S_{k+1}^{i}\right\| \\
& \leq\left\|T_{a_{1}^{i} \chi_{V^{c}}} \ldots T_{a_{k-1}^{i} \chi_{V^{c}}}\right\|\left\|\left(T_{a_{k}^{i}}-T_{a_{k}^{i} \chi_{V^{c}}}\right) S_{k+1}^{i}\right\| \\
& \leq\left\|T_{a_{k}^{i} \chi_{V}} S_{k+1}^{i}\right\|<\varepsilon,
\end{aligned}
$$

which leads to

$$
\begin{aligned}
& \left\|T_{a_{1}^{i}} \ldots T_{a_{n_{i}}^{i}} T_{\chi_{U^{c}}}-T_{a_{1}^{i} \chi_{V^{c}}} \ldots T_{a_{n_{i}}^{i} \chi_{V^{c}}} T_{\chi_{U^{c}}}\right\| \\
& \quad \leq \sum_{k=1}^{n_{i}}\left\|T_{a_{1}^{i} \chi_{V^{c}}} \ldots T_{a_{k-1}^{i} \chi_{V^{c}}} S_{k}^{i}-T_{a_{1}^{i} \chi_{V^{c}}} \ldots T_{a_{k}^{i} \chi_{V} c} S_{k+1}^{i}\right\|<n_{i} \varepsilon .
\end{aligned}
$$

Therefore

$$
\left\|\left(\sum_{i=1}^{m} \prod_{j=1}^{n_{i}} T_{a_{j}^{i}}\right) T_{\chi_{U^{c}}}-\left(\sum_{i=1}^{m} \prod_{j=1}^{n_{i}} T_{a_{j}^{i} \chi_{V^{c}}}\right) T_{\chi_{U^{c}}}\right\| \leq \sum_{i=1}^{m} n_{i} \varepsilon .
$$

Since $S T_{\chi_{U^{c}}}=\left(\sum_{i=1}^{m} \prod_{j=1}^{n_{i}} T_{a_{j}^{i}}\right) T_{\chi_{U^{c}}}$ and $\varepsilon>0$ is arbitrary, the lemma follows from (6.1) and the above inequality.

If $\mathcal{B} \subset L^{\infty}(\mathbb{D})$ is a subalgebra, we write $\mathfrak{C}_{0}(\mathcal{B})$ for the bilateral ideal of $\mathfrak{T}_{0}(\mathcal{B})$ generated by commutators $\left[T_{a}, T_{b}\right]=T_{a} T_{b}-T_{b} T_{a}$, with $a, b \in \mathcal{B}$. Therefore, $\mathfrak{C}(\mathcal{B})$ is the closure of $\mathfrak{C}_{0}(\mathcal{B})$ in $\mathfrak{L}\left(L_{a}^{2}\right)$.

Lemma 6.3 Let $\mathcal{B}$ be a hyperbolic algebra. If $S \in \mathfrak{C}_{0}\left(L^{\infty}(\mathbb{D})\right)$ is such that $B_{0} S \in \mathcal{B}$ and $\widehat{B_{0} S^{\mathcal{B}}} \equiv 0$ on $\Gamma_{\mathcal{B}}$ then $S \in \mathfrak{C}(\mathcal{B})$.

Proof. By hypothesis

$$
S=\sum_{i=1}^{m} T_{b_{1}^{i}} \ldots T_{b_{n_{i}}^{i}}\left[T_{a_{1}^{i}}, T_{a_{2}^{i}}\right] T_{c_{1}^{i}} \ldots T_{c_{k_{i}}^{i}},
$$

where $n_{i}, k_{i}$ and $m$ are some positive integers and all the symbols are in $L^{\infty}(\mathbb{D})$. If $\widehat{B_{0} S^{\mathcal{B}}} \equiv 0$ on $\Gamma_{\mathcal{B}}$, Lemma 6.2 says that given $\varepsilon>0$ there are relative neighborhoods $U, V$ of $\Gamma_{\mathcal{B}}$ such that if

$$
R=\sum_{i=1}^{m} T_{b_{1}^{i} \chi_{V^{c}}} \ldots T_{b_{n_{i}}^{i} \chi_{V^{c}}}\left[T_{a_{1}^{i} \chi_{V^{c}}}, T_{a_{2}^{i} \chi_{V^{c}}}\right] T_{c_{1}^{i} \chi_{V^{c}}} \ldots T_{c_{k_{i}}^{i} \chi_{V^{c}}} T_{\chi_{U^{c}}}
$$

then $\|S-R\|<\varepsilon$. By Lemma 6.1 every Toeplitz operator involved in the last expression is in $\mathfrak{T}(\mathcal{B})$. So, $R \in \mathfrak{C}(\mathcal{B})$ and then so is $S$. 
It is well known that if $\mathcal{B}, \mathcal{D}$ are $C^{*}$-algebras and $\phi$ is a $*$-homomorphism from $\mathcal{B}$ to $\mathcal{D}$, then $\|\phi\| \leq 1$ and $\phi$ is an isometry if and only if $\phi$ is one-to-one [13, p. 100].

Theorem 6.4 If $\mathcal{B}$ is a hyperbolic algebra then

(1) $\mathfrak{C}(\mathcal{B})=\left\{S \in \mathfrak{T}(\mathcal{B}):{\widehat{B}, S^{\mathcal{B}}} \equiv 0\right.$ on $\left.\Gamma_{\mathcal{B}}\right\}=\left\{S \in \mathfrak{T}(\mathcal{B}): \widehat{S}_{y}^{\mathcal{B}}=0\right.$ for all $\left.y \in \Gamma_{\mathcal{B}}\right\}$.

(2) $S-T_{B_{0} S} \in \mathfrak{C}(\mathcal{B})$ for every $S \in \mathfrak{T}(\mathcal{B})$.

(3) The $C^{*}$-algebras $\mathfrak{T}(\mathcal{B}) / \mathfrak{C}(\mathcal{B})$ and $C\left(\Gamma_{\mathcal{B}}\right)$ are isomorphic via $\phi: S+$ $\left.\mathfrak{C}(\mathcal{B}) \mapsto \widehat{B_{0} S^{\mathcal{B}}}\right|_{\Gamma_{\mathcal{B}}}$.

Proof. (1). The equality of the last two sets follows from Corollary 4.7. Suppose first that $S \in \mathfrak{C}_{0}(\mathcal{B})$, so

$$
S=\sum_{1 \leq i \leq n} A_{i}\left[T_{a_{i}}, T_{b_{i}}\right] B_{i}
$$

where $a_{i}, b_{i} \in \mathcal{B}$ and $A_{i}, B_{i} \in \mathfrak{T}_{0}(\mathcal{B})$. If $x \in \pi^{-1}\left(\Gamma_{\mathcal{B}}\right)$ then $a_{i} \circ \varphi_{x}$ and $b_{i} \circ \varphi_{x}$ are constant functions for all $1 \leq i \leq n$. By (4.2) then

$$
S_{x}=\sum_{1 \leq i \leq n}\left(A_{i}\right)_{x}\left[T_{a_{i} \circ \varphi_{x}}, T_{b_{i} \circ \varphi_{x}}\right]\left(B_{i}\right)_{x}=0 .
$$

Since every $S \in \mathfrak{C}(\mathcal{B})$ can be approximated by operators of this form, then $S_{x}=0$ for every $x \in \pi^{-1}\left(\Gamma_{\mathcal{B}}\right)$. By Corollary 4.7 then $B_{0} S \equiv 0$ on $\pi^{-1}\left(\Gamma_{\mathcal{B}}\right)$, which is another way to say that $\widehat{B_{0} S^{\mathcal{B}}} \equiv 0$ on $\Gamma_{\mathcal{B}}$. This proves the inclusion of the first set into the second one.

Suppose now that $S \in \mathfrak{T}(\mathcal{B})$ and $\widehat{B_{0} S^{\mathcal{B}}} \equiv 0$ on $\Gamma_{\mathcal{B}}$. We can assume that $\|S\|=1$. Let $0<\varepsilon<1$ and take $Q \in \mathfrak{T}_{0}(\mathcal{B})$ such that $\|Q-S\|<\varepsilon$. Since $Q \in \mathfrak{T}(\mathcal{B})$ then $\widehat{Q}_{y}^{\mathcal{B}}=\lambda I$ and $\widehat{\left(B_{0} Q\right)^{\mathcal{B}}}(y)=\lambda$ for every $y \in \Gamma_{\mathcal{B}}$, where $\lambda \in \mathbb{C}$ depends on $y$. Thus

$$
\left(\widehat{T}_{B_{0} Q}^{\mathcal{B}}\right)_{y}=\lim _{z \rightarrow y} T_{\left(B_{0} Q\right) \circ \varphi_{z}}=T_{\widehat{\left(B_{0} Q\right)^{\mathcal{B}}}(y)}=\lambda I .
$$

Then

$$
B_{0}\left(Q-T_{B_{0} Q}\right)^{\wedge \mathcal{B}} \equiv 0 \quad \text { on } \Gamma_{B}
$$

by Corollary 4.7 , and since $\widehat{B_{0} S^{\mathcal{B}}} \equiv 0$ on $\Gamma_{B}$ then

$$
\widehat{B_{0}\left(T_{B_{0} S}\right)^{\mathcal{B}}} \equiv 0 \quad \text { on } \Gamma_{B}
$$

by the same corollary. 
So, if

$$
S_{1}=Q-T_{B_{0} Q}+T_{B_{0} S}
$$

then $\widehat{B_{0} S_{1}}{ }^{\mathcal{B}} \equiv 0$ on $\Gamma_{\mathcal{B}}$ and

$$
\left\|S_{1}-S\right\| \leq\|Q-S\|+\left\|T_{B_{0} S}-T_{B_{0} Q}\right\| \leq 2\|Q-S\|<2 \varepsilon .
$$

In $[20$, Thm. 1.1] it is proved that

$$
\mathfrak{C}\left(L^{\infty}(\mathbb{D})\right)=\mathfrak{T}\left(L^{\infty}(\mathbb{D})\right),
$$

so it contains the identity $I$.

Since Theorem 5.7 implies that $\mathfrak{C}\left(L^{\infty}(\mathbb{D})\right)=\mathfrak{C}(\mathcal{A})$ then $I \in \mathfrak{C}(\mathcal{A})$. Consequently there is $R \in \mathfrak{C}_{0}(\mathcal{A})$ such that $\|R-I\|<\varepsilon$. Thus

$$
\left\|R S_{1}-S_{1}\right\| \leq\|R-I\|\left\|S_{1}\right\|<\varepsilon(\|S\|+2 \varepsilon)<3 \varepsilon .
$$

Since $B_{0} S_{1} \equiv 0$ on $\pi^{-1}\left(\Gamma_{\mathcal{B}}\right)$, Corollary 4.7 says that $\left(S_{1}\right)_{x}=0$ for all $x \in$ $\pi^{-1}\left(\Gamma_{\mathcal{B}}\right)$. By $(4.2)$ then $\left(R S_{1}\right)_{x}=R_{x}\left(S_{1}\right)_{x}=0$ for all $x \in \pi^{-1}\left(\Gamma_{\mathcal{B}}\right)$, which means that

$$
\left.B_{0}\left(R S_{1}\right) \in \mathcal{B} \quad \text { and } \quad \widehat{B_{0}\left(R S_{1}\right.}\right)^{\mathcal{B}} \equiv 0 \quad \text { on } \Gamma_{\mathcal{B}}
$$

But since $R \in \mathfrak{C}_{0}(\mathcal{A})$ and $S_{1} \in \mathfrak{T}_{0}(\mathcal{A})$ then $R S_{1} \in \mathfrak{C}_{0}(\mathcal{A})$, which together with Lemma 6.3 gives $R S_{1} \in \mathfrak{C}(\mathcal{B})$. By (6.2) and (6.3), $\left\|R S_{1}-S\right\|<5 \varepsilon$ and (1) follows.

(2). Let $y \in \Gamma_{\mathcal{B}}$. Since $S \in \mathfrak{T}(\mathcal{B})$ then $\widehat{S}_{y}^{\mathcal{B}}=\lambda I$. Thus

$$
\widehat{\left(B_{0} S\right)^{\mathcal{B}}}(y)=\lambda \quad \text { and } \quad\left(\widehat{T}_{B_{0} S}^{\mathcal{B}}\right)_{y}=T_{\widehat{\left(B_{0} S\right)^{\mathcal{B}}}(y)}=\lambda I .
$$

The result then follows from (1).

(3). By (1) the map $\phi$ is well-defined and one-to-one. It is clear that $\phi$ is $*$-linear. Suppose that $S, T \in \mathfrak{T}(\mathcal{B})$ and $y \in \Gamma_{\mathcal{B}}$. Then

$$
\widehat{S}_{y}^{\mathcal{B}}=\lambda_{S} I \quad \text { and } \quad \widehat{T}_{y}^{\mathcal{B}}=\lambda_{T} I
$$

for some $\lambda_{S}, \lambda_{T} \in \mathbb{C}$ that depend on $y$. Hence

$$
\begin{aligned}
\left.\widehat{B_{0}(S T}\right)^{\mathcal{B}}(y) & =\lim _{z \rightarrow y}\left\langle S_{z} T_{z} 1,1\right\rangle=\left\langle\widehat{S}_{y}^{\mathcal{B}} \widehat{T}_{y}^{\mathcal{B}} 1,1\right\rangle \\
& =\left\langle\lambda_{S} \lambda_{T} 1,1\right\rangle=\lambda_{S} \lambda_{T}=\widehat{\left(B_{0} S\right)^{\mathcal{B}}}(y) \widehat{\left(B_{0} T\right)^{\mathcal{B}}}(y),
\end{aligned}
$$

and $\phi$ is multiplicative. If $f \in C\left(\Gamma_{\mathcal{B}}\right)$ we can extend $f$ to a continuous function $F$ on $M(\mathcal{B})$. Therefore $F \in \mathcal{B}$ and

$$
\phi\left(T_{F}+\mathfrak{C}(\mathcal{B})\right)=\left.{\widehat{B_{0} F}}^{\mathcal{B}}\right|_{\Gamma_{\mathcal{B}}}=f .
$$

So, $\phi$ is onto. 
Theorem 6.5 Let $\mathcal{B}$ be a hyperbolic algebra and $S \in \mathfrak{T}_{0}\left(L^{\infty}(\mathbb{D})\right)$. Then

(1) $S \in \mathfrak{T}(\mathcal{B})$ if and only if $B_{0} S \in \mathcal{B}$.

(2) $S \in \mathfrak{C}(\mathcal{B})$ if and only if $\widehat{B_{0} S^{\mathcal{B}}} \equiv 0$ on $\Gamma_{\mathcal{B}}$.

Proof. (1). We know the necessity from Theorem 4.9. Suppose that

$$
S=\sum_{i=1}^{m} \prod_{j=1}^{n_{i}} T_{a_{j}^{i}}
$$

where all $a_{j}^{i} \in L^{\infty}(\mathbb{D})$, and $B_{0} S \in \mathcal{B}$. Then $T_{B_{0} S} \in \mathfrak{T}(\mathcal{B})$ and

$$
B_{0}\left(S-T_{B_{0} S}\right)^{-\mathcal{B}} \equiv 0 \quad \text { on } \Gamma_{B} .
$$

Consequently Lemma 6.2 tells us that given $\varepsilon>0$ there are relative neighborhoods $U, V$ of $\Gamma_{\mathcal{B}}$ such that

$$
\left\|S-T_{B_{0} S}-\sum_{i=1}^{m} \prod_{j=1}^{n_{i}} T_{a_{j}^{i} \chi_{V^{c}}} T_{\chi_{U^{c}}}+T_{\left(B_{0} S\right) \chi_{V^{c}}} T_{\chi_{U^{c}}}\right\|<\varepsilon .
$$

By Lemma 6.1,

$$
T_{a_{j}^{i} \chi_{V^{c}}}, T_{\chi_{U^{c}}}, T_{\left(B_{0} S\right) \chi_{V^{c}}} \in \mathfrak{T}(\mathcal{B})
$$

for all $1 \leq i \leq m$ and $1 \leq j \leq n_{i}$. Therefore $S \in \mathfrak{T}(\mathcal{B})$.

(2). The necessity follows from (1) of Theorem 6.4. For the sufficiency, observe that it is implicit in the condition $\widehat{B_{0} S^{\mathcal{B}}} \equiv 0$ on $\Gamma_{\mathcal{B}}$ that $B_{0} S \in \mathcal{B}$. By the previous assertion then $S \in \mathfrak{T}(\mathcal{B})$. So, (1) of Theorem 6.4 says that $S \in \mathfrak{C}(\mathcal{B})$.

If $\mathcal{B}$ is a hyperbolic algebra and $a \in \mathcal{A}$, then $a \in \mathcal{B}$ if and only if $B_{0} a \in \mathcal{B}$. Therefore the theorem says that $T_{a} \in \mathfrak{T}(\mathcal{B})$ if and only if $a \in \mathcal{B}$ and that $T_{a} \in \mathfrak{C}(\mathcal{B})$ if and only if $a \equiv 0$ on $\pi^{-1}\left(\Gamma_{\mathcal{B}}\right)$.

The algebra $C(\overline{\mathbb{D}})$, of continuous functions on the closed disk is hyperbolic, its maximal ideal space identifies with $\overline{\mathbb{D}}$, and it is immediate that $\Gamma_{C(\overline{\mathbb{D}})}=\partial \mathbb{D}$ via this identification. Since by Coburn's theorem $\mathfrak{C}(C(\overline{\mathbb{D}}))$ is the ideal of compact operators, then part (2) of the theorem says that $S \in \mathfrak{T}_{0}\left(L^{\infty}(\mathbb{D})\right)$ is compact if and only if

$$
\left(B_{0} S\right)(z) \rightarrow 0 \quad \text { as }|z| \rightarrow 1^{-} .
$$

That is, we recover the theorem of Axler and Zheng [4, Thm. 2.2]. It is clear that the above condition is equivalent to $S_{x}=0$ for all $x \in M(\mathcal{A}) \backslash \mathbb{D}$, or what is the same, $S_{z} \rightarrow 0$ in the SOT-topology when $|z| \rightarrow 1$. 


\section{Applications}

\subsection{Continuous functions up to a boundary set}

Suppose that $E \subset \partial \mathbb{D}$ is a closed set and consider the algebra $C_{E}$ formed by the functions of $\mathcal{A}$ that extend continuously to $E$. Then $C_{E}$ is a hyperbolic algebra. If $i d \in \mathcal{A}$ denotes the function $i d(z)=z$ and for $\lambda \in \partial \mathbb{D}$ we write

$$
M_{\lambda}=\{x \in M(\mathcal{A}): i d(x)=\lambda\}
$$

for the fiber of $\lambda$ over $M(\mathcal{A})$, then $M\left(C_{E}\right)$ consists of $M(\mathcal{A}) / \sim$, where $\sim$ is the equivalence relation that collapses $M_{\lambda}$ to a single point (depending on $\lambda$ ) for each $\lambda \in E$. Thus, $\Gamma_{C_{E}}$ can be identified with $E$. Theorem 6.4 then says that

$$
\mathfrak{C}\left(C_{E}\right)=\left\{S \in \mathfrak{T}\left(C_{E}\right): \lim _{z \rightarrow E}\left(B_{0} S\right)(z)=0\right\} \text { and } \mathfrak{T}\left(C_{E}\right) / \mathfrak{C}\left(C_{E}\right) \simeq C(E) .
$$

As mentioned before, when $E=\partial \mathbb{D}$, the above isomorphism is part of Coburn's theorem. Now consider the algebra $C L_{E}^{\infty}$ formed by the functions in $L^{\infty}(\mathbb{D})$ that extend continuously to $E$. Since $C L_{E}^{\infty} \not \subset \mathcal{A}$, it is not a hyperbolic algebra. So, at a first sight it is not possible to apply our results to this algebra. Fortunately, Theorem 5.7 gives us a way to overcome this apparent difficulty. In fact, it is easy to prove that if $f \in C L_{E}^{\infty}$ then $B_{k} f \in$ $C_{E}$ for every $k \geq 0$ and $\left(B_{k} f\right)(\lambda)=f(\lambda)$ for $\lambda \in E$. By Theorem 5.7 then $\mathfrak{T}\left(C_{E}\right)=\mathfrak{T}\left(C L_{E}^{\infty}\right)$ and $\mathfrak{C}\left(C_{E}\right)=\mathfrak{C}\left(C L_{E}^{\infty}\right)$.

\subsection{The McDonald-Sundberg Theorem}

Let $\mathcal{U}$ be the $C^{*}$-subalgebra of $L^{\infty}(\mathbb{D})$ generated by $H^{\infty}=\left\{f \in L^{\infty}(\mathbb{D})\right.$ : $f$ is analytic $\}$. The celebrated corona theorem of Carleson [10] states that $\mathbb{D}$ is dense in $M\left(H^{\infty}\right)$, the maximal ideal space of $H^{\infty}$. This translates into the alternative description of $\mathcal{U}$ as $C\left(M\left(H^{\infty}\right)\right)$. Since Schwarz Lemma implies that $H^{\infty} \subset \mathcal{A}$ then $\mathcal{U} \subset \mathcal{A}$. Therefore $\mathcal{U}$ is a prehyperbolic algebra and we aim to prove that it is hyperbolic.

Clearly, every interpolating sequence for $H^{\infty}$ is interpolating for $\mathcal{U}$. The interpolating sequences for $H^{\infty}$ were characterized by Carleson in [9]. Suppose that $x \in M\left(H^{\infty}\right) \backslash \mathbb{D}$ is in the closure of some interpolating sequence $\left\{z_{n}\right\}$ for $H^{\infty}$, where we can assume that $z_{n} \neq 0$ for all $n \geq 1$. It is known that the infinite product

$$
b(\omega)=\prod_{n \geq 1} \frac{\left|z_{n}\right|}{z_{n}} \varphi_{z_{n}}(\omega)
$$

represents a function $b \in H^{\infty}$ such that $b\left(z_{n}\right)=0$ for all $n \geq 1$. This $b$ is called an interpolating Blaschke product. 
We also know (see $[15$, p. 404]) that if $\delta \in(0,1)$ then there is $\varepsilon(\delta)>0$ such that

$$
|b(\omega)| \geq \varepsilon(\delta) \text { for every } \omega \in \mathbb{D} \backslash \bigcup_{n \geq 1} K\left(z_{n}, \delta\right) .
$$

Thus $x$ satisfies condition $\left(\mathrm{b}_{2}\right)$ of Proposition 3.9. On the other hand, if $x \in M\left(H^{\infty}\right) \backslash \mathbb{D}$ is not in the closure of any interpolating sequence for $H^{\infty}$, it is known that for every net $\left(z_{\alpha}\right)$ in $\mathbb{D}$ that tends to $x$,

$$
f \circ \varphi_{z_{\alpha}} \rightarrow \lambda \in \mathbb{C}
$$

uniformly on compact sets for every $f \in H^{\infty}$ (see $[15, \mathrm{Ch} . \mathrm{X}]$ ). Since $\mathcal{U}$ is the $C^{*}$-algebra generated by $H^{\infty}$ the same holds for every $f \in \mathcal{U}$. Thus $x$ satisfies $\left(\mathrm{a}_{2}\right)$ of Proposition 3.8. Consequently Corollary 3.10 tells us that $\mathcal{U}$ is hyperbolic and that $\Gamma_{\mathcal{U}}$ is formed by the points $x \in M\left(H^{\infty}\right)$ that are not in the closure of any interpolating sequence for $H^{\infty}$. Such points are usually called 'trivial points' because they can be characterized as the $x \in M\left(H^{\infty}\right)$ whose Gleason part (with respect to $H^{\infty}$ ) is just $\{x\}$. For the definition and further information on Gleason parts the reader may consult the original paper of Hoffman [16] or Garnett's book [15, Ch. X].

Theorem 6.4 now tells us that $\mathfrak{T}(\mathcal{U}) / \mathfrak{C}(\mathcal{U}) \simeq C\left(\Gamma_{\mathcal{U}}\right)$, a result obtained by McDonald and Sundberg in [17]. Theorem 6.4 also says that $\mathfrak{C}(\mathcal{U})=\{S \in$ $\mathfrak{T}(\mathcal{U}):{\widehat{B_{0}}}^{\mathcal{U}} \equiv 0$ on $\left.\Gamma_{\mathcal{U}}\right\}$ and $S-T_{B_{0} S} \in \mathfrak{C}(\mathcal{U})$, which are recent additions to the McDonald-Sundberg Theorem discovered by Axler and Zheng [5].

\subsection{The algebra of nontangential limits}

Consider the algebra $\mathcal{N}=\{f \in \mathcal{A}: f$ has nontangential limits a.e. on $\partial \mathbb{D}\}$. It is clear that $\mathcal{N}$ is prehyperbolic, and we are going to use Corollary 3.10 to show that it is hyperbolic. To do so we need to characterize the interpolating sequences for $\mathcal{N}$. For $u \in \partial \mathbb{D}$ and $0<\alpha<\pi / 2$ let $\Lambda_{\alpha}(u)=\{u-\omega$ : $|\arg \omega-\arg u|<\alpha$, and $0<|u-\omega|<1\}$ be an angular region with vertex $u$ of total opening $2 \alpha$. If $V \subset \mathbb{D}$ set

$$
\mathrm{NT}_{\alpha}(V)=\left\{u \in \partial \mathbb{D}: u \in \overline{V \cap \Lambda_{\alpha}(u)}\right\} \text { and } \mathrm{NT}(V)=\bigcup_{0<\alpha<\pi / 2} \mathrm{NT}_{\alpha}(V) .
$$

Geometrically, $\mathrm{NT}(V)$ is the subset of $\partial \mathbb{D}$ that can be approached nontangentially by points of $V$. If $u \in \partial \mathbb{D}, 0<r<1$ and $0<\alpha<\pi / 2$, there is some $0<\beta<\pi / 2$ depending on $\alpha$ and $r$ such that the $r$-pseudohyperbolic neighborhood of $\Lambda_{\alpha}(u)$ is contained in $\Lambda_{\beta}(u)$. Thus

$$
\mathrm{NT}(V)=\mathrm{NT}(\{z \in \mathbb{D}: \rho(z, V) \leq r\}) .
$$

We write $|E|$ for the one-dimensional Lebesgue measure of $E \subset \partial \mathbb{D}$. 
Lemma 7.1 A separated sequence $\mathcal{S}=\left\{z_{n}\right\}$ is interpolating for $\mathcal{N}$ if and only if $|N T(\mathcal{S})|=0$. If that is the case, for any $r>0$ sufficiently small there exists $f \in \mathcal{N}$ that separates $\mathcal{S}$ from $\mathbb{D} \backslash \cup_{n \geq 1} K\left(z_{n}, r\right)$.

Proof. Suppose that $|N T(\mathcal{S})|=0$ and $\rho\left(z_{n}, z_{m}\right) \geq \delta>0$ for $n \neq m$. By (7.1) then $\mid \mathrm{NT}\left(\bigcup_{n \geq 1} K\left(z_{n}, \delta / 4\right) \mid=0\right.$. Take $f \in \mathcal{A}$ such that

$$
f\left(z_{n}\right)=1 \text { for all } n \text { and } f \equiv 0 \text { on } \mathbb{D} \backslash \bigcup_{n \geq 1} K\left(z_{n}, \delta / 4\right) .
$$

So, $f$ has null nontangential limit a.e. on $\partial \mathbb{D}$. Thus $f \in \mathcal{N}$ and separates $\mathcal{S}$ from $\mathbb{D} \backslash \bigcup_{n>1} K\left(z_{n}, \delta / 4\right)$. If $\left\{\eta_{n}\right\}$ is an arbitrary sequence and we take $g \in \mathcal{A}$ such that $g\left(z_{n}\right)=\eta_{n}$ for every $n$ then $f g \in \mathcal{N}$ and $f\left(z_{n}\right) g\left(z_{n}\right)=\eta_{n}$ for every $n$. So, $\mathcal{S}$ is interpolating for $\mathcal{N}$.

Now suppose that $|\mathrm{NT}(\mathcal{S})|>0$. If $0<\alpha_{k}<\alpha_{k+1} \rightarrow \pi / 2$ is a strictly increasing sequence, then $\mathrm{NT}(\mathcal{S})=\bigcup_{k} \mathrm{NT}_{\alpha_{k}}(\mathcal{S})$. So, there is some $\alpha_{k}=$ $\alpha$ such that $\left|\mathrm{NT}_{\alpha}(\mathcal{S})\right|>0$, and consequently there exists a compact set

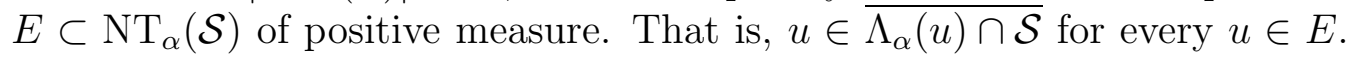
So, if $u \in E$ there is some $z_{n} \in \Lambda_{\alpha}(u) \cap \mathcal{S}$. Since $\Lambda_{\alpha}(u)$ is open, it is geometrically clear that there is a an open neighborhood $I_{u}$ of $u$ in $\partial \mathbb{D}$ such that $z_{n} \in \Lambda_{\alpha}(v) \cap \mathcal{S}$ for every $v \in I_{u}$. By the compactness of $E$ there is a finite set $\mathcal{R}_{1}$ in $\mathcal{S}$ such that $\Lambda_{\alpha}(u) \cap \mathcal{R}_{1} \neq \emptyset$ for every $u \in E$. If $r_{1}=\max \left\{|z|: z \in \mathcal{R}_{1}\right\}$ and $\mathcal{S}_{1}=\left\{z \in \mathcal{S}:|z| \leq r_{1}\right\}$ then we also have $\Lambda_{\alpha}(u) \cap \mathcal{S}_{1} \neq \emptyset$ for every $u \in E$. We can repeat this process with $\mathcal{S} \backslash \mathcal{S}_{1}$ instead of $\mathcal{S}$ to obtain $r_{2} \in\left(r_{1}, 1\right)$ such that if $\mathcal{S}_{2}=\left\{z \in \mathcal{S}: r_{1}<|z| \leq r_{2}\right\}$ then $\Lambda_{\alpha}(u) \cap \mathcal{S}_{2} \neq \emptyset$ for every $u \in E$. We keep going to construct a sequence $0<r_{1}<\cdots<r_{n}<\cdots<1$ such that if $\mathcal{S}_{n}=\left\{z \in \mathcal{S}: r_{n-1}<|z| \leq r_{n}\right\}$ then

$$
\Lambda_{\alpha}(u) \cap \mathcal{S}_{n} \neq \emptyset \text { for every } u \in E .
$$

The sequence $\left\{r_{n}\right\}$ must tend to 1 because if $r_{n} \leq r<1$ for every $n$ then $\{z:|z| \leq r\} \cap \mathcal{S}$ is infinite, which is not possible because $\mathcal{S}$ is separated. Now take

$$
\mathcal{T}_{1}=\bigcup_{j \text { odd }} \mathcal{S}_{j} \text { and } \mathcal{T}_{2}=\bigcup_{j \text { even }} \mathcal{S}_{j}
$$

Since (7.2) holds for all $n \geq 1$ then $E \subset \mathrm{NT}_{\alpha}\left(\mathcal{T}_{1}\right) \cap \mathrm{NT}_{\alpha}\left(\mathcal{T}_{2}\right)$, and since $|E|>0$, the interpolation problem

$$
f\left(z_{n}\right)= \begin{cases}1 & \text { for } z_{n} \in \mathcal{T}_{1} \\ 0 & \text { for } z_{n} \in \mathcal{T}_{2}\end{cases}
$$

cannot be solved by a function with nontangential limits almost everywhere on $E$. 
Theorem 7.2 The algebra $\mathcal{N}$ is hyperbolic. In addition, $y \in M(\mathcal{N})$ is in $G_{\mathcal{N}}$ if and only if $y$ is in the closure of some interpolating sequence for $\mathcal{N}$.

Proof. Let $y \in M(\mathcal{N})$. If $y$ is in the closure of an interpolating sequence for $\mathcal{N}$ the previous lemma says that $y$ satisfies condition $\left(\mathrm{b}_{2}\right)$ of Proposition 3.9 , so $y \in G_{\mathcal{N}}$.

If $y$ is not in the closure of an interpolating sequence for $\mathcal{N}$ and $\mathcal{S}$ is a separated sequence with $y \in \overline{\mathcal{S}}^{M(\mathcal{N})}$ then Lemma 7.1 says that $|\operatorname{NT}(\mathcal{S})|>0$. So, if $f \in \mathcal{N}$ there must be some point $u \in \mathrm{NT}(\mathcal{S})$ such that $f$ has nontangential

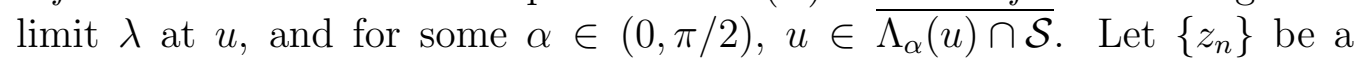
subsequence in $\Lambda_{\alpha}(u) \cap \mathcal{S}$ that tends to $u$. If $0<r<1$ then the argument preceding (7.1) says that there is some $\beta=\beta(\alpha, r) \in(0, \pi / 2)$ such that

$$
\bigcup_{n} K\left(z_{n}, r\right) \subset \Lambda_{\beta}(u)
$$

So, $f\left(\varphi_{z_{n}}(\omega)\right) \rightarrow \lambda$ for $|\omega| \leq r$ when $n \rightarrow \infty$. Thus $y$ satisfies $\left(a_{3}\right)$ of Proposition 3.8, and consequently $y \in \Gamma_{\mathcal{N}}$. By Corollary 3.10 then $\mathcal{N}$ is hyperbolic.

The nontangential limit function of $f \in \mathcal{N}$ will be denoted $\tilde{f}$. So, $\tilde{f} \in L^{\infty}(\partial \mathbb{D})$. Also, we write $z \stackrel{\text { nt }}{\rightarrow} u$ to indicate that $z$ tends nontangentially to $u \in \partial \mathbb{D}$.

Lemma 7.3 Let $f \in \mathcal{N}$. Then $\widehat{f}^{\mathcal{N}} \equiv 0$ on $\Gamma_{\mathcal{N}}$ if and only if $\tilde{f}=0$.

Proof. If there is $y \in \Gamma_{\mathcal{N}}$ such that $\left|\widehat{f}^{\mathcal{N}}(y)\right|=\delta>0$ and $\mathcal{S}$ is a separated sequence such that $y \in \overline{\mathcal{S}}^{M(\mathcal{N})}$, then $y$ is in the $M(\mathcal{N})$-closure of

$$
\mathcal{S}_{1}=\{z \in \mathcal{S}:|f(z)|>\delta / 2\}
$$

Since $y \in \Gamma_{\mathcal{N}}$ then Theorem 7.2 and Lemma 7.1 imply that $\left|\mathrm{NT}\left(\mathcal{S}_{1}\right)\right|>0$, and since $|\tilde{f}| \geq \delta / 2$ for almost every point of $\mathrm{NT}\left(\mathcal{S}_{1}\right)$, the sufficiency holds.

Now suppose that $\tilde{f} \neq 0$, so there is some $\delta>0$ such that $|\tilde{f}|>\delta$ on a set of positive measure. It is easy then to construct a separated sequence $\mathcal{S}$ such that $|\operatorname{NT}(\mathcal{S})|>0$ and $|f(z)|>\delta / 2$ for every $z \in \mathcal{S}$. The necessity will follow if we show that $\overline{\mathcal{S}}^{M(\mathcal{N})} \cap \Gamma_{\mathcal{N}} \neq \emptyset$, because for any $y$ in the intersection we would have $\left|\widehat{f}^{\mathcal{N}}(y)\right| \geq \delta / 2$.

Since $\mathcal{N}$ is hyperbolic, if $\overline{\mathcal{S}}^{M(\mathcal{N})} \cap \Gamma_{\mathcal{N}}=\emptyset$ then $\overline{\mathcal{S}}^{M(\mathcal{N})} \subset G_{\mathcal{N}}$. So, Proposition 3.9 says that for every $y \in \overline{\mathcal{S}}^{M(\mathcal{N})} \backslash \mathcal{S}$ there is an interpolating sequence $\mathcal{T}_{y}$ for $\mathcal{N}$, such that $y \in \overline{\mathcal{T}}_{y}^{M(\mathcal{N})}$. Hence, for every $0<r<1$ the $M(\mathcal{N})$ closure of $\bigcup_{z \in \mathcal{T}_{y}} K(z, r)$ is a neighborhood of $y$ (by Lemma 7.1). By the 
compactness of $\overline{\mathcal{S}}^{M(\mathcal{N})} \backslash \mathcal{S}$ there are finitely many interpolating sequences $\mathcal{T}_{1}, \ldots, \mathcal{T}_{N}$ for $\mathcal{N}$ such that the closure of

$$
U \stackrel{\text { def }}{=} \bigcup_{1 \leq j \leq N} \bigcup_{z \in \mathcal{T}_{j}} K(z, r)
$$

is a neighborhood of $\overline{\mathcal{S}}^{M(\mathcal{N})} \backslash \mathcal{S}$. Thus there is $0<\varrho<1$ so that $\mathcal{S} \cap\{z \in$ $\mathbb{D}:|z| \geq \varrho\}$ is contained in $U$. Together with (7.1) this yields

$$
\mathrm{NT}(\mathcal{S}) \subset \bigcup_{1 \leq j \leq N} \mathrm{NT}\left(\bigcup_{z \in \mathcal{T}_{j}} K(z, r)\right)=\bigcup_{1 \leq j \leq N} \mathrm{NT}\left(\mathcal{T}_{j}\right)
$$

which is impossible because $|\mathrm{NT}(\mathcal{S})|>0$ while $\left|\mathrm{NT}\left(\mathcal{T}_{j}\right)\right|=0$ for $j=1, \ldots, N$.

Lemma 7.4 If $S \in \mathfrak{T}(\mathcal{N})$ then for almost every $u \in \partial \mathbb{D}$ there is $\lambda(u) \in \mathbb{C}$ such that $S_{z} \stackrel{S O T}{\rightarrow} \lambda(u) I$ when $z \stackrel{n t}{\rightarrow} u$.

Proof. Let $a \in \mathcal{N}$ and suppose that $u \in \partial \mathbb{D}$ is such that $a(z) \rightarrow \lambda \in \mathbb{C}$ when $z \stackrel{\text { nt }}{\rightarrow} u$. If $0<\alpha<\pi / 2$ and $0<r<1$ there is $\beta=\beta(\alpha, r)$ in $(\alpha, \pi / 2)$ such that $\varphi_{z}(\omega) \in \Lambda_{\beta}(u)$ when $z \in \Lambda_{\alpha}(u)$ and $|\omega| \leq r$. Therefore $a \circ \varphi_{z} \rightarrow \lambda$ uniformly on $r \mathbb{D}$ when $z \rightarrow u$ inside $\Lambda_{\alpha}(u)$. Since $r$ is arbitrary the convergence is uniform on compact sets, implying that $\left(T_{a}\right)_{z}=T_{a \circ \varphi_{z}} \rightarrow \lambda I$ in the SOT-topology when $z \rightarrow u$ inside $\Lambda_{\alpha}(u)$. Since $\alpha$ is arbitrary and the product of operators is continuous with respect to the SOT-topology, the lemma holds for every $S \in \mathfrak{T}_{0}(\mathcal{N})$. If $S \in \mathfrak{T}(\mathcal{N})$ take a sequence $\left\{S_{n}\right\}$ in $\mathfrak{T}_{0}(\mathcal{N})$ that converges to $S$. So, for every $n \geq 1$ there is a set $E_{n} \subset \partial \mathbb{D}$ of full measure such that

$$
\left(S_{n}\right)_{z} \stackrel{\text { SOT }}{\longrightarrow} \lambda_{n}(u) I \quad \text { when } \quad z \stackrel{\text { nt }}{\longrightarrow} u \in E_{n} .
$$

Therefore the set $E=\cap E_{n}$ has full measure, and given $\varepsilon>0$ there is $n_{0}=n_{0}(\varepsilon)$ such that if $u \in E$,

$$
\left|\lambda_{n}(u)-\lambda_{m}(u)\right| \leq \lim _{z \stackrel{\text { nt }}{\longrightarrow} u}\left\|\left(S_{n}\right)_{z}-\left(S_{m}\right)_{z}\right\|=\left\|S_{n}-S_{m}\right\|<\varepsilon
$$

for all $n, m \geq n_{0}$. This implies that there is some $\lambda(u) \in \mathbb{C}$ such that $\lambda_{n}(u) \rightarrow \lambda(u)$ for every $u \in E$. If $f \in L_{a}^{2}$ has norm $1, u \in E$ and $n \geq n_{0}$, (7.3) yields

$$
\begin{aligned}
& \left\|S_{z} f-\lambda(u) f\right\| \leq\left\|S_{z} f-\left(S_{n}\right)_{z} f\right\|+\left\|\left(S_{n}\right)_{z} f-\lambda_{n}(u) f\right\|+\left|\lambda_{n}(u)-\lambda(u)\right|\|f\| \\
& \quad \leq\left\|S-S_{n}\right\|+\left|\lambda_{n}(u)-\lambda(u)\right|+\left\|\left(S_{n}\right)_{z} f-\lambda_{n}(u) f\right\| \\
& \quad \leq 2 \varepsilon+\left\|\left(S_{n}\right)_{z} f-\lambda_{n}(u) f\right\| \rightarrow 2 \varepsilon
\end{aligned}
$$

when $z \stackrel{\text { nt }}{\rightarrow} u$. Thus $S_{z} f \rightarrow \lambda(u) f$ in $L_{a}^{2}$ when $z \stackrel{\text { nt }}{\rightarrow} u \in E$ and the lemma holds for $S$. 
Theorem $7.5 \mathfrak{T}(\mathcal{N}) / \mathfrak{C}(\mathcal{N}) \simeq L^{\infty}(\partial \mathbb{D})$ and

$$
\begin{aligned}
\mathfrak{C}(\mathcal{N}) & =\left\{S \in \mathfrak{T}(\mathcal{N}): \widetilde{B_{0} S}=0\right\} \\
& =\left\{S \in \mathfrak{T}(\mathcal{N}): S_{z} \stackrel{S O T}{\longrightarrow} 0, \text { when } z \stackrel{n t}{\rightarrow} \text { u for a.e. } u \in \partial \mathbb{D}\right\} .
\end{aligned}
$$

Proof. Equality (7.4) follows immediately from Theorem 6.4 and Lemma 7.3.

By Lemma 7.4, for every $S \in \mathfrak{T}(\mathcal{N})$ there is a set $E_{S} \subset \partial \mathbb{D}$ of full measure and $\lambda_{S}: E_{S} \rightarrow \mathbb{C}$ such that

$$
S_{z} \stackrel{\mathrm{SOT}}{\rightarrow} \lambda_{S}(u) I \quad \text { when } \quad z \stackrel{\mathrm{nt}}{\rightarrow} u \in E_{S}
$$

Then $\left(B_{0} S\right)(z)=\left(B_{0} S_{z}\right)(0)=\left\langle S_{z} 1,1\right\rangle \rightarrow \lambda_{S}(u)$ when $z \stackrel{\text { nt }}{\rightarrow} u \in E_{S}$, which means that $\left(\widetilde{B_{0} S}\right)(u)=\lambda_{S}(u)$ for every $u \in E_{S}$. This proves $(7.5)$.

Let $\Phi: \mathfrak{T}(\mathcal{N}) / \mathfrak{C}(\mathcal{N}) \rightarrow L^{\infty}(\partial \mathbb{D})$ given by $\Phi(S+\mathfrak{C}(\mathcal{N}))=\widetilde{B_{0} S}$. By $(7.4)$ $\Phi$ is well-defined and one-to-one. It is also clear that $\Phi$ is $*$-linear. To prove that $\Phi$ is multiplicative let $S, T \in \mathfrak{T}(\mathcal{N})$ and use (7.6) to obtain

$$
\widetilde{B_{0}(S T)}(u)=\lim _{z \stackrel{\text { nt }}{\rightarrow}\langle}\left\langle S_{z} T_{z} 1,1\right\rangle=\lambda_{S}(u) \lambda_{T}(u)=\left(\widetilde{B_{0} S}\right)(u)\left(\widetilde{B_{0} T}\right)(u)
$$

for every $u \in E_{S} \cap E_{T}$. Hence $\phi$ is a $*$-homomorphism and we only need to show that it is onto. Let $a \in L^{\infty}(\partial \mathbb{D})$ and consider the Poisson integral

$$
A(z)=\frac{1}{2 \pi} \int_{0}^{2 \pi} \frac{1-|z|^{2}}{\left|1-z e^{-i t}\right|^{2}} a\left(e^{i t}\right) d t .
$$

So, $A$ is a bounded harmonic function such that $\tilde{A}=a$. Since $A$ is uniformly continuous with respect to $\rho$ then $A \in \mathcal{N}$. So, $T_{A} \in \mathfrak{T}(\mathcal{N})$ and

$$
\Phi\left(T_{A}+\mathfrak{C}(\mathcal{N})\right)=\widetilde{B_{0} T_{A}}=\widetilde{B_{0} A}=\tilde{A}=a .
$$

Let $\mathcal{U}$ be the algebra of the McDonald-Sundberg Theorem. Since every $f \in H^{\infty}$ has nontangential limits a.e. then $\mathcal{U} \subset \mathcal{N} \subset \mathcal{A}$. Therefore

$$
\mathfrak{C}(\mathcal{U}) \subset \mathfrak{C}(\mathcal{N}) \subset \mathfrak{C}(\mathcal{A})
$$

We shall show that both inclusions are proper. The function

$$
a=\sin \left(\log \frac{1+|z|}{1-|z|}\right)
$$

is in $\mathcal{A}$ but has no nontangential limit at any point of $\partial \mathbb{D}[8]$. Hence,

$$
T_{a} \in \mathfrak{C}(\mathcal{A}) \backslash \mathfrak{T}(\mathcal{N})
$$


The Shilov boundary of $H^{\infty}$, denoted $\partial H^{\infty}$, is the smallest closed set $F \subset M\left(H^{\infty}\right)$ such that

$$
\|f\|_{\infty}=\sup _{x \in F}\left|\widehat{f}^{\mathcal{U}}(x)\right| \quad \text { for every } f \in H^{\infty} .
$$

It is known that $\partial H^{\infty}$ is properly contained in $\Gamma_{\mathcal{U}}[15, \mathrm{p} .438]$, and that a function $f \in \mathcal{U}$ satisfies $\widehat{f}^{\mathcal{U}} \equiv 0$ on $\partial H^{\infty}$ if and only if its nontangential function vanishes a.e. on $\partial \mathbb{D}$ (see [3, Thm.7] and [7, Coro. 1.3]). So, take $y \in \Gamma_{\mathcal{U}} \backslash \partial H^{\infty}$ and $f \in \mathcal{U}$ such that $\widehat{f}^{\mathcal{U}} \equiv 0$ on $\partial H^{\infty}$ and $\widehat{f}^{\mathcal{U}}(y)=1$. Since $f(z)$ has trivial nontangential limits almost everywhere then $T_{f} \in \mathfrak{C}(\mathcal{N})$ but since $\widehat{f}^{\mathcal{U}} \not \equiv 0$ on $\Gamma_{\mathcal{U}}$ then $T_{f} \notin \mathfrak{C}(\mathcal{U})$.

Let $\mathcal{N} L^{\infty}$ be the algebra of functions in $L^{\infty}(\mathbb{D})$ that have nontangential limits a.e. on $\partial \mathbb{D}$. From the paragraph preceding (7.1) it easily follows that if $f \in \mathcal{N} L^{\infty}$ then $B_{k} f$ has the same nontangential limits as $f$ a.e. on $\partial \mathbb{D}$ for every $k \geq 0$. Thus Theorem 5.7 tells us that

$$
\mathfrak{T}(\mathcal{N})=\mathfrak{T}\left(\mathcal{N} L^{\infty}\right) \quad \text { and } \quad \mathfrak{C}(\mathcal{N})=\mathfrak{C}\left(\mathcal{N} L^{\infty}\right) .
$$

Moreover, let $E \subset \mathbb{D}$ be a set of positive measure. Then all of the above can be generalized (with similar proofs) for the algebras

$$
\mathcal{N} L_{E}^{\infty}=\left\{f \in L^{\infty}(\mathbb{D}): f \text { has nontangential limits a.e. on } E\right\}
$$

and

$$
\mathcal{N}_{E}=\mathcal{N} L_{E}^{\infty} \cap \mathcal{A}
$$

Hence, we obtain a version of Theorem 7.5 , where $\mathcal{N}$ is replaced by $\mathcal{N}_{E}$ or $\mathcal{N} L_{E}^{\infty}$ and $\partial \mathbb{D}$ is replaced by $E$.

\subsection{Constant on hyperbolic parts}

Definition. If $F \subset M(\mathcal{A}) \backslash \mathbb{D}$ is a closed saturated set, define

$$
\mathrm{CO}(F)=\left\{f \in \mathcal{A}:\left.f\right|_{F}=\text { const. }\right\} \text {. }
$$

and

$$
\operatorname{COH}(F)=\left\{f \in \mathcal{A}:\left.f\right|_{H(x)}=\text { const. for every } x \in F\right\} .
$$

These notations stand for 'constant on $F$ ' and 'constant on hyperbolic parts of $F^{\prime}$, respectively. It is clear that $\mathrm{CO}(F)$ and $\mathrm{COH}(F)$ are hyperbolic algebras and that

$$
F=\pi_{1}^{-1}\left(\Gamma_{\mathrm{CO}(F)}\right)=\pi_{2}^{-1}\left(\Gamma_{\mathrm{COH}(F)}\right),
$$

where $\pi_{1}$ and $\pi_{2}$ are the projections from $M(\mathcal{A})$ onto the respective maximal ideal spaces. 
If $\mathcal{B}$ is a hyperbolic algebra and $\pi: M(\mathcal{A}) \rightarrow M(\mathcal{B})$ is the usual projection then

$$
\left\{S \in \mathfrak{T}_{0}(\mathcal{A}):\left.B_{0} S\right|_{\pi^{-1}\left(\Gamma_{\mathcal{B}}\right)}=0\right\} \subset \mathfrak{C}(\mathcal{B}) \subset\left\{S \in \mathfrak{T}(\mathcal{A}):\left.B_{0} S\right|_{\pi^{-1}\left(\Gamma_{\mathcal{B}}\right)}=0\right\}
$$

where the first inclusion follows from Theorem 6.5 and the second from Theorem 6.4. Observe that since the first set contains $\mathfrak{C}_{0}(\mathcal{B})$, it is dense in $\mathfrak{C}(\mathcal{B})$. The significance of $\mathrm{CO}(F)$ and $\mathrm{COH}(F)$ is given by the following

Proposition 7.6 Let $\mathcal{B}$ be a hyperbolic algebra and $F \subset M(\mathcal{A})$ be a closed saturated set. Then the following conditions are equivalent

(1) $F=\pi^{-1}\left(\Gamma_{\mathcal{B}}\right)$,

(2) $\mathfrak{C}(\mathcal{B})=\mathfrak{C}(\mathrm{COH}(F))$,

(3) $\mathrm{CO}(F) \subset \mathcal{B} \subset \mathrm{COH}(F)$.

Proof. We prove first the equivalence between (1) and (2). If (1) holds then the comment following $(7.7)$ says that $\left\{S \in \mathfrak{T}_{0}(\mathcal{A}):\left.B_{0} S\right|_{F}=0\right\}$ is dense in both $\mathfrak{C}(\mathcal{B})$ and $\mathfrak{C}(\mathrm{COH}(F))$, so they must coincide. If (2) holds, (7.7) implies that

$$
\left\{S \in \mathfrak{T}_{0}(\mathcal{A}):\left.B_{0} S\right|_{\pi^{-1}\left(\Gamma_{\mathcal{B}}\right)}=0\right\} \subset\left\{S \in \mathfrak{T}(\mathcal{A}):\left.B_{0} S\right|_{F}=0\right\} .
$$

Therefore $F \subset \pi^{-1}\left(\Gamma_{\mathcal{B}}\right)$, and a symmetrical argument gives the other inclusion, so (1) holds.

If (1) holds the functions of $\mathrm{CO}(F)$ are continuous on $M(\mathcal{B})$ and the functions of $\mathcal{B}$ are continuous on $M(\mathrm{COH}(F))$. Since these are all $C^{*}$-algebras, (3) holds. If (3) holds then

$$
\mathfrak{C}(\mathrm{CO}(F)) \subset \mathfrak{C}(\mathcal{B}) \subset \mathfrak{C}(\mathrm{COH}(F))
$$

so the proof of (2) reduces to show that $\mathfrak{C}(\mathrm{CO}(F))=\mathfrak{C}(\mathrm{COH}(F))$. But this equality is a special case of the equivalence between (1) and (2).

Let us write $\mathrm{COH}$ for $\mathrm{COH}(M(\mathcal{A}) \backslash \mathbb{D})$. In this case the last proposition says that $\mathfrak{C}(\mathrm{COH})=\mathfrak{C}(C(\overline{\mathbb{D}}))$, and this is the ideal of compact operators $\mathcal{K}$. Then Theorem 6.4 tells us that $S-T_{B_{0} S} \in \mathcal{K}$ for every $S \in \mathfrak{T}(\mathrm{COH})$. In particular,

$$
\mathfrak{T}(\mathrm{COH}) / \mathcal{K}=\left\{T_{b}+\mathcal{K}: b \in \mathrm{COH}\right\} .
$$

The center of an algebra $\mathcal{B}$ is formed by the elements that commute with all the members of $\mathcal{B}$. Our next result relates $\mathfrak{T}(\mathrm{COH}) / \mathcal{K}$ with the center of $\mathfrak{T}\left(L^{\infty}(\mathbb{D})\right) / \mathcal{K}$. 
Suppose that $S \in \mathcal{K}$ and for $z \in \mathbb{D}$ let $k_{z}^{0}=\left(1-|z|^{2}\right) K_{z}^{(0)}$. Since $\left\|k_{z}^{0}\right\|=1$ and $k_{z}^{0} \rightarrow 0$ weakly as $|z| \rightarrow 1$, then

$$
\left|\left(B_{0} S\right)(z)\right| \leq\left\|S k_{z}^{0}\right\| \rightarrow 0 \quad \text { when }|z| \rightarrow 1 .
$$

Therefore $S_{x}=0$ for every $x \in M(\mathcal{A}) \backslash \mathbb{D}$.

Theorem 7.7 Let $\mathfrak{I}=\left\{S \in \mathfrak{T}\left(L^{\infty}(\mathbb{D})\right): S_{x}=0\right.$ for $\left.x \in M(\mathcal{A}) \backslash \mathbb{D}\right\}$. Then

$$
\left\{T_{b}+\mathcal{K}: b \in \mathrm{COH}\right\} \subset \operatorname{Center}\left(\mathfrak{T}\left(L^{\infty}(\mathbb{D})\right) / \mathcal{K}\right) \subset\left\{T_{b}+\mathfrak{I}: b \in \mathrm{COH}\right\}
$$

Proof. We prove first that if $S \in \mathfrak{T}\left(L^{\infty}(\mathbb{D})\right)$ and $b \in \mathrm{COH}$ then $\left[S, T_{b}\right] \in \mathcal{K}$. Let $S_{n} \in \mathfrak{T}_{0}(\mathcal{A})$ such that $S_{n} \rightarrow S$. Since $\left(S_{n} T_{b}-T_{b} S_{n}\right) \rightarrow\left(S T_{b}-T_{b} S\right)$ we can assume that $S \in \mathfrak{T}_{0}(\mathcal{A})$. By (4.2),

$$
\left(S T_{b}-T_{b} S\right)_{x}=S_{x}\left(T_{b}\right)_{x}-\left(T_{b}\right)_{x} S_{x} \text { for every } x \in M(\mathcal{A}),
$$

and since $\left(T_{b}\right)_{x}$ is a constant operator for every $x \in M(\mathcal{A}) \backslash \mathbb{D}$, then

$$
\left[S, T_{b}\right]_{x}=0 \quad \text { for } x \in M(\mathcal{A}) \backslash \mathbb{D} .
$$

The comment after Theorem 6.5 then says that $\left[S, T_{b}\right]$ is compact. This proves that $\left\{T_{b}+\mathcal{K}: b \in \mathrm{COH}\right\}$ is contained in the center of $\mathfrak{T}\left(L^{\infty}(\mathbb{D})\right) / \mathcal{K}$.

Now suppose that $S \in \mathfrak{T}\left(L^{\infty}(\mathbb{D})\right)$ is such that

$$
S+\mathcal{K} \subset \operatorname{Center}\left(\mathfrak{T}\left(L^{\infty}(\mathbb{D})\right) / \mathcal{K}\right) .
$$

This means that $S T_{a}-T_{a} S \in \mathcal{K}$ for every $a \in L^{\infty}(\mathbb{D})$. So,

$$
S_{x}\left(T_{a}\right)_{x}-\left(T_{a}\right)_{x} S_{x}=0 \quad \text { for every } x \in M(\mathcal{A}) \backslash \mathbb{D},
$$

or equivalently,

$$
S_{z}\left(T_{a}\right)_{z}-\left(T_{a}\right)_{z} S_{z} \stackrel{\text { SOT }}{\rightarrow} 0 \text { as }|z| \rightarrow 1
$$

Let $x \in M(\mathcal{A}) \backslash \mathbb{D}$ and take a net $\left(z_{\alpha}\right)$ in $\mathbb{D}$ converging to $x$. The closed ball of center 0 and radius $\|S\|$ in $\mathfrak{L}\left(L_{a}^{2}\right)$ admits a metric $d$ with the SOTtopology. Since $S_{z_{\alpha}} \stackrel{\text { SOT }}{\longrightarrow} S_{x}$ then for every integer $n \geq 1$ there is some point of the net, that we rename as $z_{n}$, such that $d\left(S_{z_{n}}, S_{x}\right)<1 / n$. So,

$$
S_{z_{n}} \stackrel{\mathrm{SOT}}{\longrightarrow} S_{x}
$$

If $\left\{r_{n}\right\}$ is a sequence in $(0,1)$ that tends to 1 , we can assume (taking a subsequence of $\left\{z_{n}\right\}$ if needed) that $K\left(z_{n}, r_{n}\right) \cap K\left(z_{j}, r_{j}\right)=\emptyset$ if $n \neq j$. For an arbitrary $a \in L^{\infty}(\mathbb{D})$ consider the function

$$
b(\omega)=\sum_{j \geq 1}\left(a \circ \varphi_{z_{j}}\right)(\omega) \chi_{K\left(z_{j}, r_{j}\right)}(\omega) .
$$


Hence $\left(T_{b}\right)_{z_{n}}=T_{b \circ \varphi_{z_{n}}}$, where

$$
\begin{aligned}
\left(b \circ \varphi_{z_{n}}\right)(\omega) & =a(\omega) \chi_{K\left(0, r_{n}\right)}(\omega)+\sum_{j: j \neq n}\left(a \circ \varphi_{z_{j}}\right)\left(\varphi_{z_{n}}(\omega)\right) \chi_{K\left(\varphi_{z_{n}}\left(z_{j}\right), r_{j}\right)}(\omega) \\
& =g_{n}(\omega)+h_{n}(\omega) .
\end{aligned}
$$

Since the support of $h_{n}$ is disjoint from $K\left(0, r_{n}\right)=r_{n} \mathbb{D}$ then

$$
\left|h_{n}(\omega)\right| \leq\|a\|_{\infty} \chi_{D \backslash r_{n} D}(\omega) \quad \text { for all } \omega \in \mathbb{D} \text {. }
$$

Since $r_{n} \rightarrow 1$, it is clear that $T_{h_{n}} \stackrel{\text { SOT }}{\rightarrow} 0$ and $T_{g_{n}} \stackrel{\text { SOT }}{\rightarrow} T_{a}$. Thus

$$
\left(T_{b}\right)_{z_{n}}=T_{g_{n}}+T_{h_{n}} \stackrel{\mathrm{SOT}}{\rightarrow} T_{a}
$$

By (7.8)

$$
S_{z_{n}}\left(T_{a}\right)_{z_{n}}-\left(T_{a}\right)_{z_{n}} S_{z_{n}} \stackrel{\text { SOT }}{\rightarrow} 0,
$$

which together with (7.9) and (7.10) gives $S_{x} T_{a}-T_{a} S_{x}=0$. This means that $S_{x}$ commutes with every Toeplitz operator with symbol in $L^{\infty}(\mathbb{D})$. By [12, Thm. 10.28] then $S_{x}=\lambda I$ for some $\lambda \in \mathbb{C}$, and consequently $B_{0} S \equiv$ $\lambda$ on $H(x)$ by Corollary 4.7. Since $x \in M(\mathcal{A}) \backslash \mathbb{D}$ is arbitrary then $B_{0} S \in$ $\mathrm{COH}$ and

$$
\left(S-T_{B_{0} S}\right)_{x}=S_{x}-T_{\left(B_{0} S\right) \circ \varphi_{x}}=\lambda I-\lambda I=0
$$

for every $x \in M(\mathcal{A}) \backslash \mathbb{D}$. That is, $S-T_{B_{0} S} \in \mathfrak{I}$.

The concept of center plays an important role when studying localizations of $C^{*}$-algebras (see [13, Th. 7.47]). I believe that the ideal $\mathfrak{I}$ in Theorem 7.7 is $\mathcal{K}$, so the inclusions of the theorem should be equalities. If $S \in \mathfrak{L}\left(L_{a}^{2}\right)$, the essential spectrum $\sigma_{e}(S)$ is the spectrum of $S+\mathcal{K}$ in the Calkin algebra $\mathfrak{L}\left(L_{a}^{2}\right) / \mathcal{K}$. Let $\sigma(S)$ denote the usual spectrum of $S$. Is it true that

$$
\sigma_{e}(S)=\bigcup_{x \in M(\mathcal{A}) \backslash \mathbb{D}} \sigma\left(S_{x}\right) \text { for every } S \in \mathfrak{T}\left(L^{\infty}(\mathbb{D})\right) ?
$$

There is strong evidence to support an affirmative answer. This holds for $S \in \mathfrak{T}(\mathrm{COH})$, while the example preceding Lemma 4.8 shows that this fails for a general $S \in \mathfrak{L}\left(L_{a}^{2}\right)$. This example appeared in [4], where it is also shown that there is an infinite dimensional orthogonal projection $P$ such that $B_{0} P(z) \rightarrow 0$ when $|z| \rightarrow 1$. We do not know the answer even for a general Toeplitz operator with bounded symbol.

Acknowledgement. I thank Manuel Flores for many interesting discussions regarding the Berezin transforms. 


\section{References}

[1] Ahern, P. And Čučković, Ž.: A theorem of Brown-Halmos type for Bergman space Toeplitz operators. J. Funct. Anal. 187 (2001), 200-210.

[2] Ahern, P., Flores, M. And Rudin, W.: An invariant volume-meanvalue property. J. Funct. Anal. 111 (1993), 380-397.

[3] Axler, S. And Shields, A.: Extensions of harmonic and analytic functions. Pacific J. Math. 145 (1990), 1-15.

[4] Axler, S. And Zheng, D.: Compact operators via the Berezin transform Indiana Univ. Math. J. 47 (1998), no. 2, 387-400.

[5] Axler, S. And Zheng, D.: The Berezin transform on the Toeplitz algebra. Studia Math. 127 (1998), no. 2, 113-136.

[6] Berezin, F. A.: Covariant and contravariant symbols of operators. (Russian). Izv. Akad. Nauk SSSR Ser. Mat. 36 (1972), 1134-1167.

[7] Bishop, C. J.: Some characterizations of $C(\mathcal{M})$. Proc. Amer. Math. Soc. 124 (1996), 2695-2701.

[8] Brudnyi, A.: Topology of the maximal ideal space of $H^{\infty}$. J. Funct. Anal. 189 (2002), no. 1, 21-52.

[9] Carleson, L.: An interpolationproblem for bounded analytic functions. Amer. J. Math. 80 (1958), 921-930.

[10] Carleson, L.: Interpolations by bounded analytic functions and the corona problem. Ann. of Math. (2) 76 (1962), 547-559.

[11] Coburn, L. A.: Singular integral operators and Toeplitz operators on odd spheres. Indiana Univ. Math. J. 23 (1973), 433-439.

[12] Conway, J. B.: Subnormal operators. Research Notes in Mathematics 51. Pitman, Boston-London, 1981.

[13] Douglas, R. G.: Banach algebra techniques in operator theory. Graduate Texts in Mathematics 179. Springer-Verlag, New York, 1998.

[14] Engliš, M.: Toeplitz operators on Bergman-type spaces. Ph.D. Thesis, Prague (1991).

[15] Garnett, J. B.: Bounded analytic functions. Pure and Applied Mathematics 96. Academic Press, Inc., New York-London, 1981.

[16] Hoffman, K.: Bounded analytic functions and Gleason parts. Ann. of Math. (2) 86 (1967), 74-111.

[17] McDonald, G. And Sundberg, C.: Toeplitz operators on the disc. Indiana Univ. Math. J. 28 (1979), 595-611.

[18] NikoL'SKII, N. K.: Treatise on the shift operator. Spectral function theory. Grundlehren der Mathematischen Wissenschaften 273. Springer-Verlag, Berlin, 1986.

[19] Stroethoff, K.: The Berezin transform and operators on spaces of analytic functions. In Linear operators (Warsaw, 1994), 361-380. Banach Center Publ. 38. Polish Acad. Sci., Warsaw, 1997. 
[20] SuÁrez, D.: The Toeplitz algebra on the Bergman space coincides with its commutator ideal. J. Operator Theory 51 (2004), no. 1, 105-114.

[21] Zhu, K. H.: Operator theory in function spaces. Monographs and Textbooks in Pure and Applied Mathematics 139. Marcel Dekker, Inc., New York, 1990.

Recibido: 20 de noviembre de 2001

Daniel Suárez

Departament de Matemàtiques Universitat Autònoma de Barcelona 08193, Bellaterra, Barcelona (Spain) dsuarez@mat .uab.es 